\title{
Gravitational self-force on a particle in eccentric orbit around a Schwarzschild black hole
}

\author{
Leor Barack and Norichika Sago \\ School of Mathematics, University of Southampton, Southampton, SO17 1BJ, United Kingdom, \\ Yukawa Institute for Theoretical Physics, Kyoto University, Kyoto 606-8502, Japan
}

(Dated: March 16, 2010)

\begin{abstract}
We present a numerical code for calculating the local gravitational self-force acting on a pointlike particle in a generic (bound) geodesic orbit around a Schwarzschild black hole. The calculation is carried out in the Lorenz gauge: For a given geodesic orbit, we decompose the Lorenz-gauge metric perturbation equations (sourced by the delta-function particle) into tensorial harmonics, and solve for each harmonic using numerical evolution in the time domain (in $1+1$ dimensions). The physical self-force along the orbit is then obtained via mode-sum regularization. The total self-force contains a dissipative piece as well as a conservative piece, and we describe a simple method for disentangling these two pieces in a time-domain framework. The dissipative component is responsible for the loss of orbital energy and angular momentum through gravitational radiation; as a test of our code we demonstrate that the work done by the dissipative component of the computed force is precisely balanced by the asymptotic fluxes of energy and angular momentum, which we extract independently from the wave-zone numerical solutions. The conservative piece of the self-force does not affect the time-averaged rate of energy and angular-momentum loss, but it influences the evolution of the orbital phases; this piece is calculated here for the first time in eccentric strong-field orbits. As a first concrete application of our code we recently reported the value of the shift in the location and frequency of the innermost stable circular orbit due to the conservative self-force [Phys. Rev. Lett. 102, 191101 (2009)]. Here we provide full details of this analysis, and discuss future applications.
\end{abstract}

\section{INTRODUCTION}

The prospects for detecting gravitational waves from the inspiral of compact objects into massive black holes have motivated, over the past decade, research in effort to understand the general-relativistic orbital evolution in such systems. The underlying elementary theoretical problem is that of a pointlike mass particle in a strong-field orbit around a Kerr black hole of a much larger mass. The dynamics of such systems can be described in a perturbative fashion in terms of an effective gravitational self-force (SF) [1 [5]; knowledge of this force is a prerequisite for describing the precise evolution of the orbit and the phasing of the emitted gravitational waves. There is an active research program focused on the development of computational methods and actual working codes for the SF in Kerr spacetime [6]. This research agenda is being pursued in incremental steps, through exploration of a set of simplified model problems with increasing complexity and physical relevance. Much of the initial work has concentrated on a scalarfield toy model $7-13$, but more recently workers have begun to tackle the gravitational case [14 18$]$. The state of the art is represented by three independent calculations of the gravitational SF for circular geodesic orbits in Schwarzschild geometry [15, 17, 18]. These calculations use different analytic and numerical methods (and they even invoke different physical interpretations of the SF), but they were shown to be fully consistent with each other [18, 19]. These calculations were also shown to be consistent with results from post-Newtonian theory in the weak-field limit [17, 20, 21].

In the current work we extend the analysis of Ref. 15] (hereafter "Paper I") from the special class of circular geodesics to generic (bound) geodesics of the Schwarzschild geometry. This generalization is astrophysically relevant because real inspirals often remain quite eccentric up until the eventual plunge into the massive hole [22]. At a more fundamental level, the generalization to eccentric orbits is interesting because it allows us to start exploring in earnest the conservative effects of the $\mathrm{SF}$ - for instance, how it influences the orbital precession. Eccentric orbits have already been considered in calculations of the scalar [12] and electromagnetic (EM) 23] SFs by Haas. While these calculations are of a less direct astrophysical relevance, they offer an important test-ground for computational techniques potentially applicable in the gravitational problem too. Indeed, many elements of our numerical method take their inspiration from Haas' work.

The numerical code we present here takes as input the two orbital parameters of an eccentric Schwarzschild geodesic (the semi-latus rectum and eccentricity, to be defined below), and returns the value of the Lorenz-gauge gravitational SF along this geodesic. The dissipative and conservative pieces of the SF are returned separately. Here we do not consider the evolution of the orbit under the effect of the SF, but leave this important next step for future work. We envisage using, to this end, a version of the "osculating geodesics" method 24], which takes as input the value of the $\mathrm{SF}$ along geodesics tangent to the actual inspiral orbit. A systematic framework for analyzing the long-term evolution of the inspiral orbits, using multiple-scale perturbation methods, was recently developed by Hinderer and Flanagan 
[25] (cf. Sec. VII of Gralla and Wald [4]).

Our strategy is similar to that of Paper I. Its basic elements are (i) the Lorenz-gauge perturbation formalism of Barack and Lousto [26], (ii) a finite-difference algorithm for numerical integration of the Lorenz-gauge perturbation equations in the time domain, and (iii) mode-sum regularization [27 30]. The perturbation formalism is based on a tensor-harmonic decomposition of the perturbed Einstein equations in the Lorenz gauge. The equations are augmented with "gauge damping" terms designed to suppress gauge violations [26], and are written as a set of 10 hyperbolic equations (for certain linear combinations of metric components) which do not couple at their principal parts. These equations are sourced by the (tensor-harmonic modes of the) particle's energy-momentum, modeled with a deltafunction distribution along the specified eccentric geodesic. The equations are solved numerically mode by mode in the time domain using characteristic coordinates on a uniform 1+1-dimensions mesh. The non-radiative monopole and dipole modes cannot be evolved stably in this manner; instead, we solve for these two modes separately in the frequency domain, using the recently introduced "extended homogeneous solutions" technique [31] to cure the irregularity of the Fourier sum near the particle. The code records the value of the perturbation modes and their derivatives along the orbit (each mode has a $C^{0}$ behavior at the particle and hence a well-defined value there, as well as a well-defined "one-sided" derivatives). These values are then fed into the "mode-sum formula" 29], which returns the physical SF through mode-by-mode regularization.

One of the primary advantages of the time-domain approach is that eccentric orbits - even ones with large eccentricity - are essentially "as easy" to deal with as circular orbits, with computational cost being only a weak function of the eccentricity [32]. Also, a time-domain code for circular orbits can be upgraded with relative ease to accommodate eccentric orbits (such a generalization is radically less straightforward in the frequency domain). Still, there are several important technical issues which arise in the time-domain upgrade from circular to eccentric orbits, and need to be addressed. We list some of these issues below.

- Most obvious, the computational burden increases significantly because the parameter space for geodesics turns from 1D (circular) to 2D (eccentric). Moreover, for each given geodesic parameters the SF becomes a function along the orbit (it has a constant value along a circular geodesic), and one is required to obtain this function over an entire radial period. The latter becomes a technical hurdle in situations where the radial period is very large - e.g., close to the last stable orbit, or for orbits with very large radii.

- In paper I we were able to improve the convergence rate of our finite-difference algorithm using a Richardson-type extrapolation to the limit of a vanishing numerical grid-cell size. That was possible because in the circular-orbit case the numerical mesh could be easily arranged such that the local discretization error varied smoothly along the orbit. This cannot be achieved in any simple way when the orbit is eccentric, and as a result one cannot implement a similar Richardson extrapolation. The practical upshot is that one is forced to implement a higherorder finite-difference scheme: a 2nd-order-convergent algorithm (as in Paper I) proves insufficient in practice. For this work we developed an algorithm with a 4th-order global convergence. The algorithm takes a rather complicated form near the particle's trajectory, where the field (the Lorenz-gauge metric perturbation) has discontinuous derivatives. To somewhat lessen this complexity (and reduce the number of grid points needed as input for the finite-difference formula) the algorithm makes use of suitable junction conditions across the orbit. The eventual numerical scheme is considerably more sophisticated - and involved - compared to that of Paper I.

- In the mode-sum scheme one first calculates the contribution to the "full" (pre-regularization) force from each tensorial-harmonic mode of the perturbation, and then decomposes this into spherical harmonics. The necessary input data for the mode-sum formula are the individual spherical-harmonic contributions. This procedure involves the implementation of a tensor-scalar coupling formula, whose details depend on the orbit in question. The coupling formula simplifies considerably in the circular-orbit case; it reverts to its full complicated form [Eq. (2.25) with Appendix [] when eccentric orbits are considered.

- The computation of the monopole and dipole contributions to the SF (which we perform in the frequency domain, as mentioned above) becomes much more involved in the eccentric-orbit case. First, the spectrum of the orbital motion now includes all harmonics of the radial frequency, and one has to calculate and add up sufficiently many of these harmonics. A second, more technically challenging complication arises from the fact that the perturbation becomes a non-smooth function of time across the orbit (at a given radius), which disrupts the high-frequency convergence of the Fourier sum at the particle (a behavior reminiscent of the Gibbs phenomenon). A general method for circumventing this problem in frequency-domain calculations was devised recently in Ref. [31], and we implement it here for the first time.

- In exploring the physical consequences of the SF it is useful to split the SF into its dissipative and conservative pieces, and discuss their corresponding effects in separate. This splitting is straightforward in the circular-orbit 
case: The conservative piece is precisely the (Schwarzschild) $r$ component of the SF, while the (Schwarzschild) $t, \varphi$ components exactly account for the entire dissipative effect. This is no longer true for eccentric orbits, where each of the Schwarzschild components mixes up both dissipative and conservative pieces, and it is not immediately obvious how to extract these pieces individually. Here we suggest and implement a simple new method for constructing the dissipative and conservative pieces out of the computed Schwarzschild components of the SF (without resorting to a calculation of the advanced perturbation). The method takes advantage of the general symmetries of Schwarzschild geodesics.

With the computational framework in place, we can start to explore the physical effects of the gravitational SF. In this article we concentrate on two such effects. First, we calculate the loss of orbital energy and angular momentum, over one radial period, due to the dissipative piece of the SF. We extract these quantities directly from the computed SF along the geodesic orbit (for a sample of orbital parameters). These "lost" energy and angular momentum must be balanced by the total amount of energy and angular momentum in the gravitational waves radiated to spatial infinity and into the black hole over a radial period. We derive formulas for extracting these quantities from the far-zone and near-horizon numerical Lorenz-gauge solutions, and demonstrate numerically that they agree well with the values computed from the local SF. Our values for the energy and angular momentum losses also agree with those previously obtained by others using other methods.

The second effect we consider is conservative, and cannot be inferred indirectly from the asymptotic gravitational waves: It is the conservative shift in the location and frequency of the Innermost Stable Circular Orbit (ISCO). The analysis of the ISCO shift requires knowledge of the SF along slightly eccentric geodesics near the last stable orbit, and our code provides the necessary SF data for the first time. We reported the results in a recent Letter [33], and here we describe our analysis in full detail. The quantitative determination of the ISCO shift is important in that it provides a strong-field benchmark for calibration of approximate (e.g., post-Newtonian) descriptions of binary inspirals. Our result for the ISCO frequency shift has already been incorporated by Lousto et al. in their "empirical" fitting formula for predicting the remnant mass and spin parameters in binary mergers [34, 35]; and by Damour [21] for breaking the degeneracy between certain unknown parameters of the Effective One Body (EOB) formalism.

Perhaps of a more direct relevance to the problem of the phase evolution in binaries with extreme mass-ratio is the effect of the SF on the periapsis precession of the eccentric orbit-also a conservative effect. SF corrections to the precession rate have been analyzed for weak-field orbits and within the toy model of the EM SF [36, 37], but never before for the gravitational problem in strong field. Our code generates the SF data necessary to tackle this problem for the first time. We leave the detailed analysis of SF precession effects to a forthcoming paper.

The paper is organized as follows. In Sec. II we review the relevant theoretical background: bound geodesics in Schwarzschild geometry, the Lorenz-gauge metric perturbation formulation, and the construction of the SF via the mode-sum formula. Section III describes our numerical method in detail, and in Sec. IV we present numerical results for a few sample eccentric orbits, including a "zoom-whirl" orbit. We explain how the dissipative and conservative pieces of the computed SF can be extracted from the numerical data, and present these two pieces separately in a few sample cases. We also analyze the dissipative effect of the SF and demonstrate the consistency between the dissipated energy and angular momentum inferred from the local SF, and that extracted from the asymptotic gravitational waves. Section V covers the ISCO-shift analysis, and in Sec. VI we summarize and discuss future applications of our code.

Throughout this work we use standard geometrized units (with $c=G=1$ ), metric signature -+++ , and (unless indicated otherwise) Schwarzschild coordinates $x^{\mu}=(t, r, \theta, \varphi)$.

\section{THEORETICAL BACKGROUND}

\section{A. Eccentric geodesics in Schwarzschild geometry}

In this work we consider a pointlike particle with mass $\mu$ in a bound orbit around a Schwarzschild black hole of mass $M \gg \mu$. In the limit $\mu \rightarrow 0$ the trajectory of the particle is a timelike geodesic of the background Schwarzschild spacetime. We parameterize this geodesic by proper time $\tau$, in the form $x_{\mathrm{p}}^{\mu}(\tau)=\left(t_{\mathrm{p}}(\tau), r_{\mathrm{p}}(\tau), \theta_{\mathrm{p}}(\tau), \varphi_{\mathrm{p}}(\tau)\right)$, with corresponding four-velocity $u^{\mu}=d x_{\mathrm{p}}^{\mu} / d \tau$. Without loss of generality we take $\theta_{\mathrm{p}}(\tau)=\pi / 2$. The geodesic equations of the particle are given in integrated form as

$$
\begin{gathered}
\frac{d t_{\mathrm{p}}}{d \tau}=\frac{\mathcal{E}}{f\left(r_{\mathrm{p}}\right)}, \quad \frac{d \varphi_{\mathrm{p}}}{d \tau}=\frac{\mathcal{L}}{r_{\mathrm{p}}^{2}} \\
\left(\frac{d r_{\mathrm{p}}}{d \tau}\right)^{2}=\mathcal{E}^{2}-R\left(r_{\mathrm{p}}, \mathcal{L}^{2}\right), \quad R\left(r, \mathcal{L}^{2}\right) \equiv f(r)\left(1+\frac{\mathcal{L}^{2}}{r^{2}}\right),
\end{gathered}
$$


where $f \equiv 1-2 M / r$, and $\mathcal{E} \equiv-u_{t}$ and $\mathcal{L} \equiv u_{\varphi}$ are the integrals of motion corresponding to the particle's specific energy and angular momentum.

When $\mathcal{L}^{2}>12 M^{2}$ the effective potential of the radial motion, $R\left(r, \mathcal{L}^{2}\right)$, has a maximum and a minimum and hence eccentric (bound) orbits exist. These orbits can be parametrized by the two values of $r_{\mathrm{p}}$ at the turning points, $r_{\min }$ and $r_{\max }$ ("periastron" and "apastron", respectively). We may alternatively parameterize the orbits by the (dimensionless) semi-latus rectum $p$ and eccentricity $e$, defined through

$$
p \equiv \frac{2 r_{\min } r_{\max }}{M\left(r_{\min }+r_{\max }\right)}, \quad e \equiv \frac{r_{\max }-r_{\min }}{r_{\max }+r_{\min }}
$$

From the two conditions $R\left(r_{\min }\right)=R\left(r_{\max }\right)=\mathcal{E}^{2}$, one readily obtains

$$
\mathcal{E}^{2}=\frac{(p-2-2 e)(p-2+2 e)}{p\left(p-3-e^{2}\right)}, \quad \mathcal{L}^{2}=\frac{p^{2} M^{2}}{p-3-e^{2}} .
$$

Bound geodesics have $0 \leq e<1$ and $p>6+2 e$ [38]. Points along the separatrix $p=6+2 e$ (where $\mathcal{E}^{2}$ equals the maximum of the effective potential) represent marginally unstable orbits. Stable circular orbits are those with $e=0$ and $p \geq 6$, for which $\mathcal{E}^{2}$ equals the minimum of the effective potential. The point $(p, e)=(6,0)$ in the $e-p$ plane, where the separatrix intersects the $e=0$ axis, is referred to as the innermost stable circular orbit (ISCO)-see Fig. 1 .

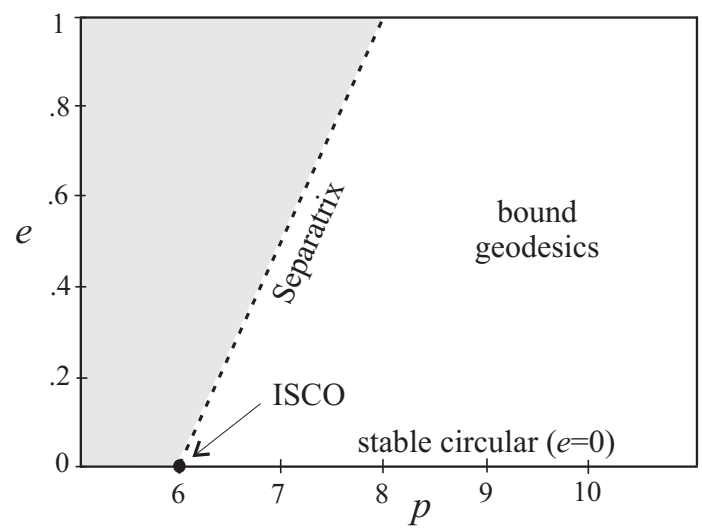

FIG. 1: Parameter space for bound geodesics in Schwarzschild spacetime. The (dimensionless) "semi-latus rectum" $p$ and "eccentricity" $e$ are defined in Eq. (2.3). Bound geodesics have $e \geq 0$ and $p>6+2 e$. Points along the separatrix $p=6+2 e$ represent marginally unstable orbits. Stable circular orbits lie along the axis $e=0$ for $p \geq 6$. The point $(p, e)=(6,0)$ is the ISCO.

Following Ref. [38], we introduce the monotonically increasing "radial phase" parameter $\chi$, defined so that the radial motion obeys

$$
r_{\mathrm{p}}(\chi)=\frac{p M}{1+e \cos \chi} .
$$

Note $\chi=2 \pi n$ ( $n$ integer) correspond to periastron passages. In terms of $\chi$, the $t$ and $\varphi$ components of the geodesic equations (2.1) are re-expressed as

$$
\begin{aligned}
\frac{d t_{\mathrm{p}}}{d \chi} & =\frac{M p^{2}}{(p-2-2 e \cos \chi)(1+e \cos \chi)^{2}} \sqrt{\frac{(p-2-2 e)(p-2+2 e)}{p-6-2 e \cos \chi}}, \\
\frac{d \varphi_{\mathrm{p}}}{d \chi} & =\sqrt{\frac{p}{p-6-2 e \cos \chi}}
\end{aligned}
$$

and the radial velocity reads

$$
u^{r}=\mathcal{E} e \sin \chi \sqrt{\frac{p-6-2 e \cos \chi}{(p-2-2 e)(p-2+2 e)}} .
$$


The period of the radial motion can be derived by integrating Eq. (2.6) with respect to $\chi$ :

$$
T_{r} \equiv \int_{0}^{2 \pi} \frac{d t_{\mathrm{p}}}{d \chi} d \chi
$$

With the initial conditions $t_{\mathrm{p}}=\varphi_{\mathrm{p}}=0$ at $\chi=0$, the particle's geodesic trajectory is fully specified by Eqs. (2.5), (2.6) and (2.7). The functions $t_{\mathrm{p}}(\chi)$ and $\varphi_{\mathrm{p}}(\chi)$ cannot be written explicitly in analytic form, but it is easy to obtain them numerically, for given $p$ and $e$, at any desired accuracy.

\section{B. Gravitational self-force via mode-sum regularization}

When $\mu$ is finite (yet still much smaller than $M$ ), the particle experiences a gravitational SF, $F^{\alpha}\left[\sim O\left(\mu^{2}\right)\right]$, and the equation of motion is formally given by

$$
\mu \frac{D^{2} \tilde{x}_{\mathrm{p}}^{\alpha}}{D \tilde{\tau}^{2}}=\mu \frac{D \tilde{u}^{\alpha}}{D \tilde{\tau}}=F^{\alpha}(\tilde{\tau})
$$

Here we use $\tilde{x}_{\mathrm{p}}^{\alpha}(\tilde{\tau})$ to denote the (non-geodesic) trajectory under the effect of the SF, with $\tilde{\tau}$ representing proper time along this trajectory and $\tilde{u}_{\mathrm{p}}^{\alpha} \equiv d \tilde{x}_{\mathrm{p}}^{\alpha} / d \tilde{\tau}$. The covariant derivatives $D / D \tilde{\tau}$ are taken with respect to the background geometry. From symmetry we have $F^{\theta}=0$. Furthermore, assuming the normalization $\tilde{u}_{\alpha} \tilde{u}^{\alpha}=-1$, we have the orthogonality condition $\tilde{u}_{\alpha} F^{\alpha}=0$, which interrelates the remaining 3 components of the SF. All in all, then, there are two non-trivial independent components of the SF to be determined.

In this work we do not consider the evolution of the orbit under the effect of the SF, i.e., we do not seek to obtain consistent solutions of Eq. (2.10). Rather, we are interested in calculating the value of the SF $F^{\alpha}(\tau)$ along a fixed, geodesic orbit $x_{\mathrm{p}}^{\alpha}(\tau)$, with given values of $p, e$. We envisage that the $\mathrm{SF}$ information $F^{\alpha}(\tau ; p, e)$ (calculated over the space of $p, e$ ) could be used, in a second step, to calculate the orbital evolution in situations where at any given time the orbit deviates only very slightly from a geodesic of the background, and the evolution takes place over a timescale much longer than the radial period ("adiabatic approximation"). Here, however, we concentrate on the first step, leaving the investigation of the orbital evolution for future work.

The gravitational SF acting on the particle at any a point along the geodesic $x_{\mathrm{p}}^{\alpha}(\tau ; p, e)$ is calculated using the mode-sum formula 27 29]

$$
F^{\alpha}=\sum_{l=0}^{\infty}\left[F_{\text {full }}^{\alpha l}-A_{ \pm}^{\alpha} L-B^{\alpha}\right] \equiv \sum_{l=0}^{\infty} F_{\text {reg }}^{\alpha l} .
$$

Here $L \equiv l+1 / 2$, and $F_{\text {full }}^{\alpha l}$ are the multipole modes of the "full" force field constructed from the (physical, retarded) Lorenz-gauge metric perturbation as prescribed in Sec. IID below. The subscript \pm refers to the two possible values of $F_{\text {full }}^{\alpha l}$ at $x_{\mathrm{p}}^{\alpha}$, resulting from taking one-sided radial derivatives of the metric perturbation from either $r \rightarrow r_{\mathrm{p}}^{+}$or $r \rightarrow r_{\mathrm{p}}^{-} . A_{ \pm}^{\alpha}$ and $B^{\alpha}$ are the "regularization parameters", given by [29, 30]

$$
\begin{gathered}
A_{ \pm}^{t}=\mp \frac{\mu^{2} u^{r}}{r_{\mathrm{p}}^{2} f_{\mathrm{p}} U}, \quad A_{ \pm}^{r}=\mp \frac{\mu^{2} \mathcal{E}}{r_{\mathrm{p}}^{2} U}, \quad A_{ \pm}^{\varphi}=0 \\
B^{t}=\frac{\mu^{2} \mathcal{E} u^{r}}{\pi r_{\mathrm{p}}^{2} f_{\mathrm{p}} U^{3 / 2}}[-\hat{K}(w)+2(1-U) \hat{E}(w)], \\
B^{r}=-\frac{\mu^{2}}{\pi r_{\mathrm{p}}^{2} U^{3 / 2}}\left[\left(\mathcal{E}^{2}+f_{\mathrm{p}} U\right) \hat{K}(w)-\left[2 \mathcal{E}^{2}(1-U)-f_{\mathrm{p}} U(1-2 U)\right] \hat{E}(w)\right], \\
B^{\varphi}=\frac{\mu^{2} u^{r}}{\pi \mathcal{L} r_{\mathrm{p}}^{2} \sqrt{U}}\left[\hat{K}(w)-\left(1+2 \frac{\mathcal{L}^{2}}{r_{\mathrm{p}}^{2}}\right) \hat{E}(w)\right],
\end{gathered}
$$

where $\hat{K}(w) \equiv \int_{0}^{\pi / 2}\left(1-w \sin ^{2} x\right)^{-1 / 2} d x$ and $\hat{E}(w) \equiv \int_{0}^{\pi / 2}\left(1-w \sin ^{2} x\right)^{1 / 2} d x$ are complete elliptic integrals of the first and second kind, respectively, $f_{\mathrm{p}} \equiv f\left(r_{\mathrm{p}}\right)=1-2 M / r_{\mathrm{p}}$, and

$$
w=\frac{\mathcal{L}^{2}}{r_{\mathrm{p}}^{2}+\mathcal{L}^{2}}, \quad U=1+\frac{\mathcal{L}^{2}}{r_{\mathrm{p}}^{2}} .
$$


It is important to remember that the gravitational SF [as also the trajectory $x_{\mathrm{p}}(\tau)$ itself] is a gauge-dependent entity [39]. The mode-sum formula (2.11) is formulated in the Lorenz-gauge, and requires as input the modes $F_{\text {full }}^{\alpha l}$ derived from the Lorenz-gauge metric perturbation. In our approach this perturbation is obtained by tackling the linearized Lorenz-gauge Einstein equations directly, in the time domain. We proceed by reviewing the relevant Lorenz-gauge perturbation formalism.

\section{Metric perturbation in Lorenz gauge}

Ref. [26] presented a formulation of the Lorenz-gauge perturbation equations in Schwarzschild spacetime, amenable to numerical treatment in the time domain. In Paper I we applied this formulation (with some minor modifications) in our study of circular orbits. Here we shall use the same Lorenz-gauge formulation to obtain our metric perturbation, and we describe it here as applied to generic eccentric orbits.

Let $g_{\mu \nu}$ be the metric of the background Schwarzschild geometry, and $h_{\mu \nu}$ be the physical (retarded) metric perturbation due to the particle moving on the geodesic $x_{\mathrm{p}}^{\alpha}(\tau ; p, e)$. We assume $h_{\mu \nu}$ is given in the Lorenz gauge, i.e., it satisfies

$$
\bar{h}_{\mu \nu}{ }^{\nu} \equiv Z_{\mu}=0,
$$

where $\bar{h}_{\mu \nu}=h_{\mu \nu}-(1 / 2) g_{\mu \nu} h$ is the trace-reversed perturbation. The corresponding Einstein equations, linearized in $h_{\mu \nu}$ over $g_{\mu \nu}$, take the compact form

$$
\bar{h}_{\mu \nu}{ }^{; \alpha} ; \alpha+2 R_{\mu}^{\alpha}{ }_{\mu}{ }_{\nu} \bar{h}_{\alpha \beta}=-16 \pi T_{\mu \nu},
$$

where a semicolon denotes covariant differentiation with respect to $g_{\mu \nu}$, and $h \equiv g^{\mu \nu} h_{\mu \nu}$ is the trace of $h_{\mu \nu}$. $T_{\mu \nu}$ on the right-hand side is the energy-momentum tensor associated with the particle, given by the distribution

$$
T_{\mu \nu}=\mu \int_{-\infty}^{\infty} \frac{u_{\mu} u_{\nu} \delta^{(4)}\left(x^{\alpha}-x_{\mathrm{p}}^{\alpha}\right)}{\sqrt{-g}} d \tau
$$

where $g$ is the determinant of $g_{\mu \nu}$.

It is well known that the hyperbolic set (2.16) admits a well-posed initial-value formulation, and that the gauge conditions (2.15) are satisfied automatically if only they are satisfied on the initial (Cauchy or characteristic) surface. However, in a time-domain numerical implementation of Eqs. (2.16) it is usually impossible to satisfy the gauge conditions (2.15) precisely on the initial surface, and, even if one succeeded to do so, finite differencing errors would usually lead to an uncontrollable violation of the gauge conditions (unless one somehow actively imposes the gauge conditions during the evolution). This problem, and its resolution, were discussed in Ref. [26], and we follow here the same method. To the original field equations (2.16) we add "divergence dissipation" terms, in the form

$$
\bar{h}_{\mu \nu}^{; \alpha} ; \alpha+2 R_{\mu}^{\alpha}{ }_{\mu}{ }_{\mu} \bar{h}_{\alpha \beta}+f^{\prime}\left(t_{\mu} \tilde{Z}_{\nu}+t_{\nu} \tilde{Z}_{\mu}\right)=-16 \pi T_{\mu \nu},
$$

where $t_{\mu}=\left(1, f^{-1}, 0,0\right)$ and $\tilde{Z}_{\mu}=\left(f Z_{r}, Z_{r}, Z_{\theta}, Z_{\varphi}\right)$. While the inclusion of these extra terms does not (in principle) affect the solutions of the equations, it guarantees that violations of the gauge condition are efficiently damped during the numerical time evolution. [This can be shown by considering the divergence of Eq. (2.18), noticing that this yields a hyperbolic equation for $Z_{\mu}$, with a manifest dissipation term [26].]

Owing to the spherical symmetry of the background geometry, the field equations (2.18) are separable into tensorial spherical-harmonics using the substitution

$$
\bar{h}_{\mu \nu}=\frac{\mu}{r} \sum_{l=0}^{\infty} \sum_{m=-l}^{l} \sum_{i=1}^{10} a_{l}^{(i)} \bar{h}^{(i) l m}(r, t) Y_{\mu \nu}^{(i) l m}(\theta, \varphi ; r)
$$

(and similarly for the source $T_{\mu \nu}$ ). The tensorial-harmonic basis $Y_{\mu \nu}^{(i) l m}$ and normalization factors $a_{l}^{(i)}(i=1, \ldots, 10)$ are the ones defined in Ref. [26], except that here (as also in Paper I) we take $Y_{\mu \nu}^{(3) l m} \rightarrow f(r) \times Y_{\mu \nu}^{(3) l m}$ (this modification is needed to achieve $\bar{h}^{(3) l m} \rightarrow$ const as $r \rightarrow 2 M$, in line with the behavior of all other functions $\left.\bar{h}^{(i) l m}\right)$. In Appendix A we give explicit formulae for reconstruction of the various Schwarzschild components $\bar{h}_{\mu \nu}$ out of the 10 scalar-like functions $\bar{h}^{(i) l m}(r, t)$.

The above substitution reduces the field equations (2.18) to the coupled set of 2-dimensional hyperbolic equations

$$
\square \bar{h}^{(i) l m}+\mathcal{M}_{(j)}^{(i) l} \bar{h}^{(j) l m}=S^{(i) l m} \delta\left(r-r_{\mathrm{p}}\right) \quad(i=1, \ldots, 10) .
$$


Here a box represents the 2-dimensional scalar-field wave operator

$$
\square=\partial_{u v}+V(r), \quad V(r)=\frac{f}{4 r^{2}}\left[l(l+1)+\frac{2 M}{r}\right],
$$

where $v$ and $u$ are the standard Eddington-Finkelstein null coordinates, defined through $v=t+r_{*}$ and $u=t-r_{*}$, with $r_{*}=r+2 M \ln [r /(2 M)-1]$. The terms $\mathcal{M}_{(j)}^{(i) l} \bar{h}^{(j) l m}$ (summation over $j$ implied) involve first derivatives of the $\bar{h}^{(j) l m}$ 's at most - hence the principal part of the set 2.20 is entirely contained in the term $\square \bar{h}^{(i) l m}$. $S^{(i) l m}$ are the source terms for the point particle, constructed from the tensor-harmonic coefficients of $T_{\mu \nu}$. In Appendix B we give explicit expressions for both $\mathcal{M}_{(j)}^{(i) l} \bar{h}^{(j) l m}$ and $S^{(i) l m}$. The time-radial functions $\bar{h}^{(i) l m}$ also satisfy four elliptic equations, which arise from the Lorenz gauge conditions (2.15). These relations, too, are given in Appendix B.

\section{Construction of the full force mode}

Given the Lorenz-gauge metric perturbation $\bar{h}_{\alpha \beta}$, the full force modes $F_{\text {full }}^{\alpha l}$ appearing in the mode-sum formula (2.11) are formally constructed as we now prescribe.

First, following [28], we define the "full-force field" as a tensor field at arbitrary spacetime points $x$, for a given (fixed) worldline point $x_{\mathrm{p}}$ (where the $\mathrm{SF}$ is to be calculated):

$$
F_{\text {full }}^{\alpha}\left(x ; x_{\mathrm{p}}\right)=\mu k^{\alpha \beta \gamma \delta}\left(x ; x_{\mathrm{p}}\right) \bar{h}_{\beta \gamma ; \delta} .
$$

Here the trace-reversed metric perturbation, $\bar{h}_{\alpha \beta}$, is evaluated at $x$, and

$$
k^{\alpha \beta \gamma \delta}\left(x ; x_{\mathrm{p}}\right)=g^{\alpha \delta} u^{\beta} u^{\gamma} / 2-g^{\alpha \beta} u^{\gamma} u^{\delta}-u^{\alpha} u^{\beta} u^{\gamma} u^{\delta} / 2+u^{\alpha} g^{\beta \gamma} u^{\delta} / 4+g^{\alpha \delta} g^{\beta \gamma} / 4
$$

where $g^{\alpha \delta}$ is the background metric at $x$, and $u^{\alpha}$ are the values of the contravariant Schwarzschild components of the four-velocity at $x_{\mathrm{p}}$ (treated as fixed coefficients). In principle, one can choose to extend the quantity $k^{\alpha \beta \gamma \delta}$ off the worldline in any one of many different ways (a few natural choices are discussed in Ref. [29]). The specific choice made here is advantageous in that it guarantees a finite mode-coupling in Eq. (2.25) below. Our choice of extension does not correspond to any of the choices made in [29], but it can be shown (using the methods of [29]) that the regularization parameters associated with our extension are the same as those of the "fixed contravariant components" extension defined in [29] - these are the parameters whose values we state above in Eqs. (2.12) and (2.13).

In the next step we expand $\bar{h}_{\alpha \beta}$ in tensor harmonics as in Eq. (2.19) and substitute in Eq. (2.22). Taking the limits $r \rightarrow r_{\mathrm{p}}$ and $t \rightarrow t_{\mathrm{p}}$ (but maintaining the $\theta, \varphi$ dependence), the full force takes the form

$$
\begin{aligned}
{\left[F_{\text {full }}^{\alpha}\left(\theta, \varphi ; r_{\mathrm{p}}, t_{\mathrm{p}}\right)\right]_{ \pm}=} & \frac{\mu^{2}}{r_{\mathrm{p}}^{2}} \sum_{l=0}^{\infty} \sum_{m=-l}^{l}\left\{f_{0 \pm}^{\alpha l m} Y^{l m}+f_{1 \pm}^{\alpha l m} \sin ^{2} \theta Y^{l m}+f_{2 \pm}^{\alpha l m} \cos \theta \sin \theta Y_{, \theta}^{l m}\right. \\
& +f_{3 \pm}^{\alpha l m} \sin ^{2} \theta Y_{, \theta \theta}^{l m}+f_{4 \pm}^{\alpha}\left(\cos \theta Y^{l m}-\sin \theta Y_{, \theta}^{l m}\right) \\
& \left.+f_{5 \pm}^{\alpha l m} \sin \theta Y_{, \theta}^{l m}+f_{6 \pm}^{\alpha l m} \sin ^{3} \theta Y_{, \theta}^{l m}+f_{7 \pm}^{\alpha l m} \cos \theta \sin ^{2} \theta Y_{, \theta \theta}^{l m}\right\},
\end{aligned}
$$

where $Y^{l m}(\theta, \varphi)$ are the spherical harmonics, and the coefficients $f_{n \pm}^{\alpha l m}$ are constructed from the perturbation fields $\bar{h}^{(i) l m}$ and their first $r$ and $t$ derivatives, all evaluated at $x_{\mathrm{p}}$. The labels $+/-$ correspond to taking one-sided derivatives from $r \rightarrow r_{\mathrm{p}}^{+}$or $r_{\mathrm{p}}^{-}$, respectively. The explicit expressions for the $f_{n \pm}^{\alpha l m}$ 's are shown in Appendix C]

Since the mode-sum formula (2.11) requires as input the spherical harmonic modes of the full force, we must now re-expand Eq. (2.24) in terms of spherical harmonics. With the help of the identities given in Appendix D, we obtain

$$
F_{\text {full }}^{\alpha l}=\frac{\mu^{2}}{r_{\mathrm{p}}^{2}} \sum_{m=-l}^{l} Y^{l m}\left(\theta_{\mathrm{p}}, \varphi_{\mathrm{p}}\right) \times\left\{\mathcal{F}_{(-3)}^{\alpha l-3, m}+\mathcal{F}_{(-2)}^{\alpha l-2, m}+\mathcal{F}_{(-1)}^{\alpha l-1, m}+\mathcal{F}_{(0)}^{\alpha l m}+\mathcal{F}_{(+1)}^{\alpha l+1, m}+\mathcal{F}_{(+2)}^{\alpha l+2, m}+\mathcal{F}_{(+3)}^{\alpha l+3, m}\right\}_{ \pm},
$$

where each of the functions $\mathcal{F}_{(i)}^{\alpha l m}$ is a certain linear combination of the $f_{n \pm}^{\alpha l m}$ 's (with the same $l, m$ ) - the explicit relations are given in Appendix C]. Hence, in general, a given full-force mode $F_{\text {full }}^{\alpha l}$ carries contributions from tensorialharmonic functions $\bar{h}_{l^{\prime} m}^{(i)}$ with $l-3 \leq l^{\prime} \leq l+3$. This coupling arises, of course, from the decomposition of the tensor-harmonic contributions into spherical harmonics. 


\section{E. Conservative and dissipative pieces of the SF}

In analyzing the physical consequences of the SF-as we start to do in Sec. V-it is useful to consider separately its conservative and dissipative pieces. We therefore now define these two pieces, and obtain a separate mode-sum formula for each. To this end, we first introduce the notation $F^{\alpha} \equiv F_{\text {ret }}^{\alpha}$, reminding us that the SF $F^{\alpha}$ is derived from the physical, retarded metric perturbation $\bar{h}_{\alpha \beta} \equiv \bar{h}_{\alpha \beta}^{\text {ret }}$. We then define the "advanced" SF through

$$
F_{\mathrm{adv}}^{\alpha}=\sum_{l=0}^{\infty}\left[F_{\mathrm{full} \pm}^{\alpha l}\left(\bar{h}_{\alpha \beta}^{\mathrm{adv}}\right)-A_{ \pm}^{\alpha} L-B^{\alpha}\right],
$$

where the modes $F_{\text {full } \pm}$ are constructed precisely as prescribed in the previous subsection, only this time using the multipole modes of the advanced metric perturbation, $\bar{h}_{\alpha \beta}^{\text {adv }}$. The regularization parameters $A^{\alpha}$ and $B^{\alpha}$ are the same as those given above for the retarded SF. Since $\bar{h}_{\alpha \beta}^{\text {ret }}$ and $\bar{h}_{\alpha \beta}^{\text {adv }}$ have the same local singular behavior near the particle [40, 41], the sum in Eq. (2.26) is guaranteed to converge.

Following Hinderer and Flanagan [25], we define the SF's conservative and dissipative components as the parts of the SF which are (correspondingly) symmetric and anti-symmetric under ret $\leftrightarrow$ adv:

$$
F^{\alpha}\left(\equiv F_{\text {ret }}^{\alpha}\right)=F_{\text {cons }}^{\alpha}+F_{\text {diss }}^{\alpha},
$$

where

$$
F_{\mathrm{cons}}^{\alpha} \equiv \frac{1}{2}\left(F_{\mathrm{ret}}^{\alpha}+F_{\mathrm{adv}}^{\alpha}\right), \quad F_{\mathrm{diss}}^{\alpha} \equiv \frac{1}{2}\left(F_{\mathrm{ret}}^{\alpha}-F_{\mathrm{adv}}^{\alpha}\right)
$$

Substituting from Eqs. (2.11) (with $F^{\alpha} \rightarrow F_{\text {ret }}^{\alpha}$ ) and (2.26), we obtain the mode-sum formulas

$$
\begin{gathered}
F_{\mathrm{cons}}^{\alpha}=\sum_{l=0}^{\infty}\left[F_{\text {full(cons }) \pm}^{\alpha l}-A_{ \pm}^{\alpha} L-B^{\alpha}\right] \equiv \sum_{l=0}^{\infty} F_{\mathrm{reg}(\mathrm{cons})}^{\alpha l} \\
F_{\mathrm{diss}}^{\alpha}=\sum_{l=0}^{\infty} F_{\mathrm{full}(\mathrm{diss}) \pm}^{\alpha l} \equiv \sum_{l=0}^{\infty} F_{\mathrm{reg}(\mathrm{diss})}^{\alpha l}
\end{gathered}
$$

where

$$
F_{\text {full (cons) } \pm}^{\alpha l} \equiv \frac{1}{2}\left[F_{\text {full } \pm}^{\alpha l}\left(\bar{h}_{\alpha \beta}^{\text {ret }}\right)+F_{\text {full } \pm}^{\alpha l}\left(\bar{h}_{\alpha \beta}^{\text {adv }}\right)\right], \quad F_{\text {full (diss }) \pm}^{\alpha l} \equiv \frac{1}{2}\left[F_{\text {full }}^{\alpha l}\left(\bar{h}_{\alpha \beta}^{\text {ret }}\right)-F_{\text {full }}^{\alpha l}\left(\bar{h}_{\alpha \beta}^{\mathrm{adv}}\right)\right]
$$

Notice that the dissipative piece of the SF requires no regularization within the mode-sum scheme.

In Eqs. (2.29) and (2.30) the splitting of the SF into its conservative and dissipative pieces is performed mode-bymode. This is useful in practice, because the $l$-modes of the two pieces exhibit a rather different large- $l$ behaviour: While $F_{\text {full(cons) } \pm}^{\alpha l}$ normally admit an asymptotic power series in $1 / l$ [starting at $\left.O(l)\right]$, the modes of $F_{\text {full(diss) } \pm}^{\alpha l}$ die off at large $l$ faster than any power of $l$. We will come back to this issue in Sec. IIID.

The extraction of the conservative and dissipative pieces using Eqs. (2.29) and (2.30) entails a calculation of both retarded and advanced metric perturbations. This would normally double the computation time, as it requires one to solve the perturbation equations twice, changing the boundary conditions in order to obtain $\bar{h} \alpha \beta$. Fortunately, in the case of a Schwarzschild background we can avoid this extra computational burden using a simple trick. For a given eccentric geodesic, we think of the SF as a function of $\tau$ along the orbit. Without loss of generality we take $\tau=0$ to correspond to a certain periapsis passage [i.e., $r_{\mathrm{p}}(\tau=0)=r_{\min }$ ]. Then we have the following symmetry relation, immediately following from Eq. (2.80) of [25]:

$$
F_{\mathrm{adv}}^{\alpha}(\tau)=\epsilon_{(\alpha)} F_{\text {ret }}^{\alpha}(-\tau)
$$

(no summation over $\alpha$ ), where $\epsilon_{(\alpha)}=(-1,1,1,-1)$ in Schwarzschild coordinates. This relation can be used to re-express Eqs. (2.28) in terms of the retarded $\mathrm{SF}$ alone, in the form

$$
F_{\mathrm{cons}}^{\alpha}(\tau)=\frac{1}{2}\left[F_{\mathrm{ret}}^{\alpha}(\tau)+\epsilon_{(\alpha)} F_{\mathrm{ret}}^{\alpha}(-\tau)\right], \quad F_{\mathrm{diss}}^{\alpha}(\tau)=\frac{1}{2}\left[F_{\mathrm{ret}}^{\alpha}(\tau)-\epsilon_{(\alpha)} F_{\mathrm{ret}}^{\alpha}(-\tau)\right]
$$

Similar relations are applicable to the $l$ modes $F_{\text {full(cons) } \pm}^{\alpha l}$ and $F_{\text {full(cons) } \pm}^{\alpha l}$ as well. These relations allow us to extract the conservative and dissipative pieces of the SF in practice without resorting to a calculation of the advanced perturbation: All that is required is knowledge of the physical (retarded) SF along the orbit. 63 . 


\section{NUMERICAL METHOD}

In this section we describe the numerical method used to solve the field equations (2.20) and to construct the local SF along the eccentric orbit. Our numerical scheme is basically similar to that of Paper I-we still use finite differencing on a characteristic grid in $1+1$ dimensions - but we have modified our code in several important aspects. Most importantly, we abandon the use of a Richardson extrapolation over the grid size: this technique relies crucially on the uniformity of the local discretization error along the orbit, which can no longer be guaranteed in any simple way when dealing with eccentric orbits. To accelerate the numerical convergence we have instead upgraded our finitedifference scheme from second-order to fourth-order. This introduces a significant amount of additional complexity, especially in the treatment of grid cells traversed by the particle. Our method inherits from Lousto [42] (fourth-order scheme for 1+1D evolution with a particle source) and Haas [12] (implementation for a scalar field), but we deviate from their methods in several aspects.

\section{A. Numerical domain and initial data}

Our integration domain is discreterized using a two-dimensional uniform mesh based on the double-null coordinates $v$ and $u$, as depicted in Fig. 2. The numerical evolution starts with characteristic initial data

$$
\bar{h}^{(i)}\left(u=u_{0}, v\right)=\bar{h}^{(i)}\left(u, v=v_{0}\right)=0 \text { for all } i,
$$

where the vertex $\left(v_{0}, u_{0}\right)$ corresponds to the particle's location at $t=0$ [so $v_{0}=-u_{0}=r_{* p}(t=0)$ where $\left.r_{* p}=r_{*}\left(r_{\mathrm{p}}\right)\right]$. The early stage of the evolution will be dominated by spurious radiation resulting from the imperfection of the initial data. However, as demonstrated in [26] (also in Paper I), these spurious waves damp down rapidly, and the error related to this behavior becomes negligible at late time. Our numerical algorithm monitors the residual error from spurious initial waves by comparing the $\mathrm{SF}$ values recorded at regular intervals along the orbit. We then make sure to evolve long enough for this error to drop below a set threshold. For a fractional error threshold of $10^{-4}$ in the final $\mathrm{SF}$ we find that the error from the spurious radiation can be safely ignored after $\sim 2-3 \times T_{r}$ of evolution (depending primarily on the value of $p$; larger $p$ requires a longer evolution).

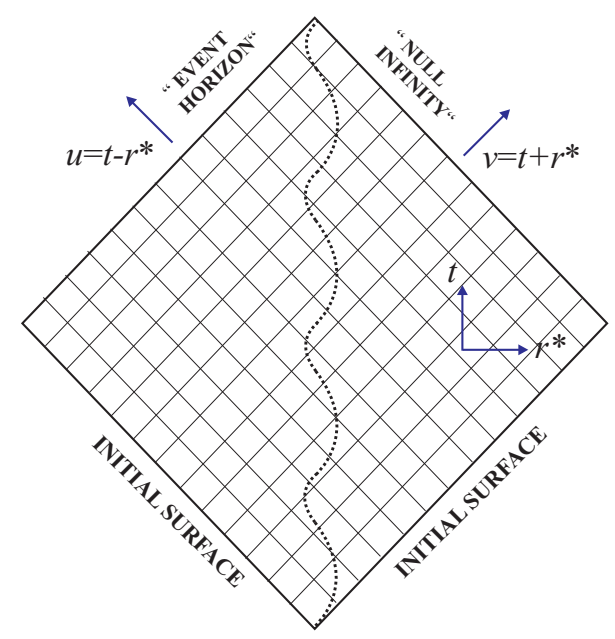

FIG. 2: Numerical domain: a staggered 1+1-dimensional mesh in null coordinates $v, u$. $r_{*}$ is the standard Schwarzschild 'tortoise' radial coordinate. The dotted line represents the trajectory of a typical eccentric orbit. In actual implementation the mesh is, of course, much finer than it is depicted here.

Note that, in our setup, the numerical domain has no causal boundaries. Therefore, no boundary conditions need be imposed.

\section{B. Finite-difference scheme}

To derive our finite-difference equations, let us focus on a grid cell of dimensions $\Delta v \times \Delta u=h \times h-$ say, the one in Fig. 3 with center $\mathrm{C}$ and vertices 1, 2, 3 and 4. We shall assume that the numerical values of $h^{(i)}$ at points $2-15$ 
are already known, and we wish to derive the value of $h^{(i)}$ at point 1 . To this end we consider the integral of the field equations (2.20) over the cell with center $\mathrm{C}$. The $u v$-derivative term on the left-hand side is integrated in exact form to give

$$
\int_{\text {cell }} \bar{h}_{, u v}^{(i)} d u d v=\bar{h}_{1}^{(i)}-\bar{h}_{2}^{(i)}-\bar{h}_{3}^{(i)}+\bar{h}_{4}^{(i)}
$$

where $\bar{h}_{n}^{(i)}$ denotes the value of $\bar{h}^{(i)}$ at the grid point labeled ' $n$ ' in Fig. 3, The integral of the source term on the right-hand side of the field equations is expressed as

$$
\mathcal{S}^{(i)} \equiv \int_{\text {cell }} S_{l m}^{(i)} \delta\left(r-r_{\mathrm{p}}\right) d u d v= \begin{cases}2 \int_{t_{i}}^{t_{f}} d t f_{\mathrm{p}}^{-1} S_{l m}^{(i)}\left(x_{\mathrm{p}}(t)\right), & \text { (orbit crosses cell) } \\ 0, & \text { (orbit outside cell) }\end{cases}
$$

where $t_{i}$ and $t_{f}$ are the values of $t$ at which, correspondingly, the particle enters and leaves the cell in question. Since the integrand on the right-hand side depends only on the known trajectory of the particle (obtained in advance by solving the geodesic equation numerically), the integral can be evaluated in exact form. Our code implements a 5-point closed Newton-Cotes formula ("Boole's rule") to evaluate this integral at each grid cell crossed by the particle.

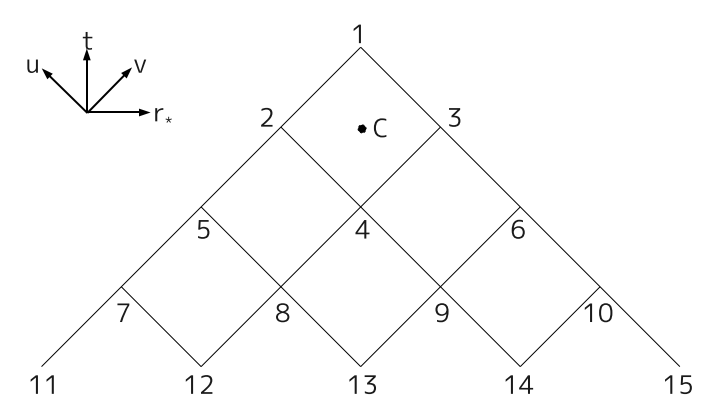

FIG. 3: Grid points involved in constructing our finite-difference scheme. The point $\mathrm{C}$ is the center of the cell over which we integrate the field equations, as described in the text. The dimensions of each grid cell are $\Delta v \times \Delta u=h \times h$.

The rest of the terms appearing in the field equations (2.20) [cf. Eqs. (B1) $-($ B10 $]$ ] can each be expressed schematically as either $H \equiv \tilde{f}(r) \bar{h}^{(j) l m}, H_{, v}$ or $H_{, r_{*}}$, where $\tilde{f}(r)$ is some known function of $r, j=1, \ldots, 10$, and the indices $(j) l m$ are suppressed for brevity. To complete the formulation of the finite-difference scheme we need to obtain finite-difference expressions for the integrals

$$
I \equiv \int_{\text {cell }} H d u d v, \quad I_{v} \equiv \int_{\text {cell }} H_{, v} d u d v, \quad \text { and } \quad I_{r_{*}} \equiv \int_{\text {cell }} H_{, r_{*}} d u d v .
$$

We are aiming here to achieve a quartic $\left[O\left(h^{4}\right)\right]$ global numerical error in the fields $\bar{h}^{(i) l m}$. Hence, the local finitedifference error for each of the integrals $I, I_{v}$ and $I_{r_{*}}$ should not exceed $O\left(h^{6}\right)$. To formulate the necessary finitedifference relations we will need to consider separately the following 3 cases (referring again to the grid cell with center C shown in Fig. 3): (1) The orbit does not cross the triangular region shown in the figure ("vacuum cell"); (2) The orbit crosses either the segment 2-11 or the segment 3-15 ("near-orbit cell"); (3) The orbit crosses either the segment 1-2 or the segment 1-3 ("orbit cell").

\section{Vacuum cell}

Consider the formal two-variable Taylor expansion of a typical term $H$ about the center of the cell in question-point $\mathrm{C}$ in Fig. 3. with coordinates $v=v_{c}$ and $u=u_{c}$. We have

$$
H(u, v)=\sum_{a+b=0}^{N} \frac{c_{a b}}{a ! b !}\left(u-u_{c}\right)^{a}\left(v-v_{c}\right)^{b}+O\left(h^{N+1}\right),
$$

where $a, b$ and $N$ are non-negative integers (the latter to be specified below), and $c_{a b}$ are constant coefficients. Since the desired error in $I$ (the integral of $H$ over the 2D cell) is $O\left(h^{6}\right)$, we are allowed an error of $O\left(h^{4}\right)$ in $H$ and hence 
take $N=3$ in Eq. (3.5). This leaves us with 10 expansion coefficients $c_{a b}$, which we can solve for, using Eq. (3.5), in terms of the values of $H$ at the 10 points $n=1-10$ indicated in Fig. 3. Substituting the values of these coefficients back in Eq. (3.5) and integrating over the grid cell, we obtain

$$
I=\frac{h^{2}}{24}\left[2 H_{1}+10\left(H_{2}+H_{3}+H_{4}\right)-4\left(H_{5}+H_{6}\right)+\left(H_{7}-H_{8}-H_{9}+H_{10}\right)\right]+O\left(h^{6}\right) \quad[\operatorname{vacuum}],
$$

where $H_{n}$ denotes the value of $H$ at grid point $n$. To evaluate $I_{v}$ and $I_{r_{*}}$ at local error $O\left(h^{6}\right)$, we need instead truncate the Taylor series (3.5) at $N=4$, now leaving us with 15 coefficients $c_{a b}$. These are determined by solving Eq. (3.5) given the values $H_{n}$ at the 15 points $n=1-15$. Taking $\partial_{v}$ and $\partial_{r_{*}}$ in Eq. (3.5) and integrating over the grid cell gives

$$
\begin{aligned}
I_{v} & =\frac{h}{24}\left[9\left(H_{1}-H_{2}\right)+19\left(H_{3}-H_{4}\right)-5\left(H_{6}-H_{9}\right)+\left(H_{10}-H_{14}\right)\right]+O\left(h^{6}\right) \quad[\operatorname{vacuum}], \\
I_{r_{*}} & =\frac{h}{24}\left[28\left(H_{3}-H_{2}\right)-5\left(H_{6}-H_{5}-H_{9}+H_{8}\right)+\left(H_{10}-H_{7}-H_{14}+H_{12}\right)\right]+O\left(h^{6}\right) \quad[\operatorname{vacuum}],
\end{aligned}
$$

which are the desired finite-difference expressions for $I_{v}$ and $I_{r_{*}}$.

\section{Near-orbit cell}

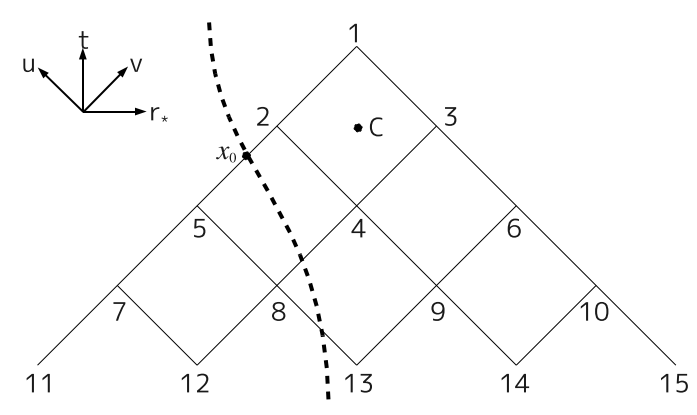

FIG. 4: Same as in Fig. 3, but now point C is located near the particle's worldline, represented by the dashed line. The finite-difference scheme for this case is described in the text under "near-orbit cell".

The above derivation assumes that the fields $\bar{h}^{(i) l m}$ (and hence $H$ ) are sufficiently smooth across the region shown in Fig. 3. This is no longer the case if the worldline of the particle traverses this region, since $H$ is generally nondifferentiable across the worldline, and the above Taylor-series-based method would fail. Let us first consider the simpler case, where the particle crosses either the segment 2-11 or the segment 3-15 (i.e., it does not traverse the integration cell itself), as demonstrated in Fig. 4. In this case the function $H(u, v)$ can still be expressed as a formal Taylor series,

$$
H(u, v)=\sum_{a+b=0}^{N} \frac{c_{a b}^{ \pm}}{a ! b !}\left(u-u_{c}\right)^{a}\left(v-v_{c}\right)^{b}+O\left(h^{(N+1)}\right)
$$

but we now have two different sets of expansion coefficients, $c_{a b}^{+}$and $c_{a b}^{-}$, depending on whether $r(u, v)>r_{\mathrm{p}}(t)$ or $r(u, v)<r_{\mathrm{p}}(t)$, respectively. As in the vacuum cell case, the value of $H$ at the grid points $1-15$ provides 15 independent equations for the unknown coefficients $c_{a b}^{ \pm}$, which, however, are now 30 in number. The necessary additional 15 relations between the various $c_{a b}^{ \pm}$'s are obtained by utilizing explicit junction conditions for $H$ and its derivatives across the particle's orbit, as we now describe.

In Appendix E we derive explicit jump conditions for the perturbation $\bar{h}^{(i) l m}$ and its derivatives at a generic point $x_{0}$ along the (known) geodesic worldline. Specifically (and using the notation of Appendix E), we calculate the two jumps $\left[\bar{h}_{, u}^{(i) l m}\right]_{0}$ and $\left[\bar{h}_{, v}^{(i) l m}\right]_{0}$, as well as the three jumps $\left[\bar{h}_{, u u}^{(i) l m}\right]_{0},\left[\bar{h}_{, u v}^{(i) l m}\right]_{0}$ and $\left[\bar{h}_{, v v}^{(i) l m}\right]_{0}$, the 4 jumps in the various third derivatives, and the 5 jumps in the various fourth derivatives. Together with the continuity condition $\left[\bar{h}^{(i) l m}\right]_{0}=0$, we hence obtain 15 jump conditions in total. Now referring back to our near-orbit cell scenario and to Fig. 4, we take $x_{0}$ to be the intersection of the worldline with the past light cone of point 1 , as demonstrated in the figure. The 15 jump conditions for $\bar{h}^{(i) l m}$ and its derivatives at $x_{0}$ readily translate into 15 jump conditions for $H$ and its derivatives 
at that point. Imposing these conditions in Eq. (3.9) yields the required additional 15 independent linear equations for the coefficients $c_{a b}^{ \pm}$. Our algorithm solves the total of 30 equations for $c_{a b}^{ \pm}$numerically, given the numerical values of $H_{n}$ at points $1-15$.

Once the coefficients $c_{a b}^{ \pm}$have been calculated, Eq. (3.9) can be integrated over the cell of centre C, giving

$$
\begin{aligned}
I & =h^{2}\left[c_{00}^{ \pm}+\frac{h^{2}}{24}\left(c_{20}^{ \pm}+c_{02}^{ \pm}\right)\right]+O\left(h^{6}\right) \quad[\text { near-orbit }] \\
I_{v} & =h^{2}\left[c_{01}^{ \pm}+\frac{h^{2}}{24}\left(c_{21}^{ \pm}+c_{03}^{ \pm}\right)\right]+O\left(h^{6}\right) \quad[\text { near-orbit }] \\
I_{r_{*}} & =h^{2}\left[c_{01}^{ \pm}-c_{10}^{ \pm}+\frac{h^{2}}{24}\left(c_{21}^{ \pm}-c_{30}^{ \pm}+c_{03}^{ \pm}-c_{12}^{ \pm}\right)\right]+O\left(h^{6}\right) \quad \text { [near-orbit] }
\end{aligned}
$$

where the values $c_{a b}^{+}$apply if $\mathrm{C}$ is located at $r>r_{\mathrm{p}}(t)$ and the values $c_{a b}^{-}$apply if $\mathrm{C}$ lies in the region $r<r_{\mathrm{p}}(t)$.

\section{Orbit cell}

The procedure described above for calculating the Taylor coefficients $c_{a b}^{ \pm}$is applicable even when the particle's worldline crosses the considered grid cell. However, the integration of $H$ and its derivatives over the grid cell then becomes slightly more involved, because the cell is divided by the trajectory into two parts, in each of which the coefficients take different values. Following Lousto [42], we consider separately the four cases illustrated in Fig. [5]

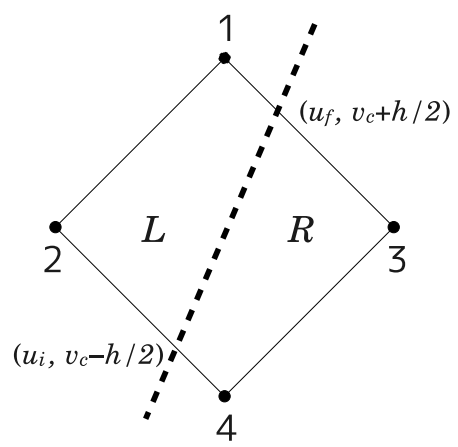

Case UU

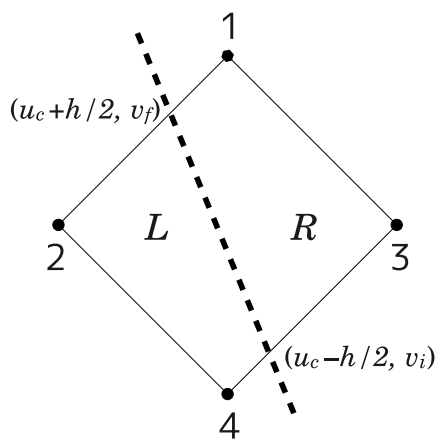

Case VV

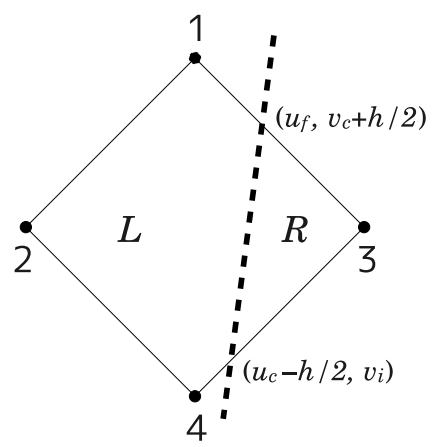

Case VU

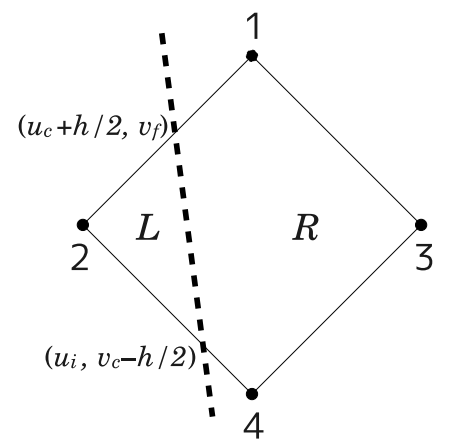

Case UV

FIG. 5: Illustration of the four cases considered in formulating the finite-difference equation for grid cells traversed by the particle's worldline (here represented by dashed lines). The center of the cell is at $(u, v)=\left(u_{c}, v_{c}\right)$, and in each case we indicate the $u, v$ coordinates of the two points where the particle enters/leaves the cell.

"Case UU" (top left in Fig. (5)): the particle enters the cell crossing the $v=$ const segment 2-4, and leaves it crossing the $v=$ const segment $1-3$. The worldline splits the cell into two bits, "left" and "right", respectively labeled $L$ and $R$ in the figure. Denoting the corresponding contributions to $I$ by $I^{L}$ and $I^{R}$, we have $I=I^{L}+I^{R}$, where, using the 
expansion (3.9),

$$
\begin{aligned}
I^{L} & =\int_{-h / 2}^{h / 2} d \bar{v} \int_{\bar{u}_{\mathrm{p}}(\bar{v})}^{h / 2} d \bar{u} H=\sum_{a+b=0}^{3} \frac{c_{a b}^{-}}{(a+1) ! b !} \int_{-h / 2}^{h / 2} d \bar{v} \bar{v}^{b}\left[(h / 2)^{a+1}-\left(\bar{u}_{\mathrm{p}}(\bar{v})\right)^{a+1}\right]+O\left(h^{6}\right), \\
I^{R} & =\int_{-h / 2}^{h / 2} d \bar{v} \int_{-h / 2}^{\bar{u}_{\mathrm{p}}(\bar{v})} d \bar{u} H=\sum_{a+b=0}^{3} \frac{c_{a b}^{+}}{(a+1) ! b !} \int_{-h / 2}^{h / 2} d \bar{v} \bar{v}^{b}\left[\left(\bar{u}_{\mathrm{p}}(\bar{v})\right)^{a+1}-(-h / 2)^{a+1}\right]+O\left(h^{6}\right),
\end{aligned}
$$

Here $\bar{u} \equiv u-u_{c}$ and $\bar{v} \equiv v-v_{c}$, and $\bar{u}_{\mathrm{p}}(\bar{v})$ represents the value of $\bar{u}$ on the trajectory, viewed as a function of $\bar{v}$. Similar expressions are easily obtained for $I_{v}$ and $I_{r_{*}}$.

"Case VU" (top right in Fig. [5): the particle enters the cell crossing the $u=$ const segment 3-4, and leaves it crossing the $v=$ const segment $1-3$. We denote the entry $v$-value by $v_{i}$ and the exit $u$-value by $u_{f}$, and further denote $\bar{v}_{i}=v_{i}-v_{c}$ and $\bar{u}_{f}=u_{f}-u_{c}$. We obtain, in this case,

$$
\begin{aligned}
I^{L}= & \int_{-h / 2}^{h / 2} d \bar{v} \int_{\bar{u}_{f}}^{h / 2} d \bar{u} H+\int_{-h / 2}^{\bar{u}_{f}} d \bar{u} \int_{-h / 2}^{\bar{v}_{\mathrm{p}}(\bar{u})} d \bar{v} H \\
= & \sum_{a+b=0}^{3} \frac{c_{a b}^{-}}{(a+1) !(b+1) !}\left\{(h / 2)^{b+1}\left[(h / 2)^{a+1}-\left(\bar{u}_{f}\right)^{a+1}\right]\left[1+(-1)^{b}\right]\right. \\
& \left.+(a+1) \int_{-h / 2}^{\bar{u}_{f}} d \bar{u} \bar{u}^{a}\left[\left(\bar{v}_{\mathrm{p}}(\bar{u})\right)^{b+1}-(-h / 2)^{b+1}\right]\right\}+O\left(h^{6}\right), \\
I^{R}= & \int_{-h / 2}^{\bar{u}_{f}} d \bar{u} \int_{\bar{v}_{\mathrm{p}}(\bar{u})}^{h / 2} d \bar{v} H=\sum_{a+b=0}^{3} \frac{c_{a b}^{+}}{a !(b+1) !} \int_{-h / 2}^{\bar{u}_{f}} d \bar{u} \bar{u}^{a}\left[(h / 2)^{b+1}-\left(\bar{v}_{\mathrm{p}}(\bar{u})\right)^{b+1}\right]+O\left(h^{6}\right),
\end{aligned}
$$

where $\bar{v}_{\mathrm{p}}(\bar{u})$ is the value of $\bar{v}$ on the trajectory, expressed as a function of $\bar{u}$. Once again, similar expressions can be obtained for $I_{v}$ and $I_{r_{*}}$.

"Case $V V$ " (bottom left in Fig. 5): the particle enters the cell crossing the $u=$ const segment 3-4, and leaves it crossing the $u=$ const segment $1-2$. In this case we obtain

$$
\begin{aligned}
I^{L} & =\int_{-h / 2}^{h / 2} d \bar{u} \int_{-h / 2}^{\bar{v}_{\mathrm{p}}(\bar{u})} d \bar{v} H=\sum_{a+b=0}^{3} \frac{c_{a b}^{-}}{a !(b+1) !} \int_{-h / 2}^{h / 2} d \bar{u} \bar{u}^{a}\left[\left(\bar{v}_{\mathrm{p}}(\bar{u})\right)^{b+1}-(-h / 2)^{b+1}\right], \\
I^{R} & =\int_{-h / 2}^{h / 2} d \bar{u} \int_{\bar{v}_{\mathrm{p}}(\bar{u})}^{h / 2} d \bar{v} H=\sum_{a+b=0}^{3} \frac{c_{a b}^{+}}{a !(b+1) !} \int_{-h / 2}^{h / 2} d \bar{u}^{a}\left[(h / 2)^{b+1}-\left(\bar{v}_{\mathrm{p}}(\bar{u})\right)^{b+1}\right],
\end{aligned}
$$

and similar expressions for $I_{v}$ and $I_{r_{*}}$.

"Case $U V$ " (bottom right in Fig. [5): the particle enters the cell crossing the $v=$ const segment 2-4, and leaves it crossing the $u=$ const segment $1-2$. We denote the entry $u$-value by $u_{i}$ and the exit $v$-value by $v_{f}$, with $\bar{u}_{i}=u_{i}-u_{c}$ and $\bar{v}_{f}=v_{f}-v_{c}$. In this final case we have

$$
\begin{aligned}
I^{L}= & \int_{-h / 2}^{\bar{v}_{f}} d \bar{v} \int_{\bar{u}_{\mathrm{p}}(\bar{v})}^{h / 2} d \bar{u} H=\sum_{a+b=0}^{3} \frac{c_{a b}^{-}}{(a+1) ! b !} \int_{-h / 2}^{\bar{v}_{f}} d \bar{v} \bar{v}^{b}\left[(h / 2)^{a+1}-\left(\bar{u}_{\mathrm{p}}(\bar{v})\right)^{a+1}\right], \\
I^{R}= & \int_{\bar{v}_{f}}^{h / 2} d \bar{v} \int_{-h / 2}^{h / 2} d \bar{u} H+\int_{-h / 2}^{\bar{v}_{f}} d \bar{v} \int_{-h / 2}^{\bar{u}_{\mathrm{p}}(\bar{v})} d \bar{u} H \\
= & \sum_{a+b=0}^{3} \frac{c_{a b}^{+}}{(a+1) !(b+1) !}\left\{(h / 2)^{a+1}\left[1+(-1)^{a}\right]\left[(h / 2)^{b+1}-\left(\bar{v}_{f}\right)^{b+1}\right]\right. \\
& \left.+(b+1) \int_{-h / 2}^{\bar{v}_{f}} d \bar{v}^{b}\left[\left(\bar{u}_{\mathrm{p}}(\bar{v})\right)^{a+1}-(-h / 2)^{a+1}\right]\right\},
\end{aligned}
$$

with similar expressions for $I_{v}$ and $I_{r_{*}}$. 


\section{Predictor-corrector method}

In summary, recalling Eq. (3.2) and with reference to Fig. 3, our basic finite-difference formula takes the form

$$
\bar{h}_{1}^{(i)}=\bar{h}_{2}^{(i)}+\bar{h}_{3}^{(i)}-\bar{h}_{4}^{(i)}-\int_{\text {cell }} d u d v\left[V(r) \bar{h}^{(i)}+\mathcal{M}_{(j)}^{(i)} \bar{h}^{(j)}\right]+\mathcal{S}^{(i)},
$$

where $\mathcal{S}^{(i)}$ is given in Eq. (3.3) and the integral (over the cell with center C in the figure) is evaluated with local error $O\left(h^{6}\right)$ as discussed above. A complication arises, since our finite-difference expressions for the integral in Eq. (3.21) involve the value of the perturbation at point 1 itself [as in, e.g., Eq. [3.6)], which is the very unknown value we wish to compute.

We overcome this difficulty using a type of predictor-corrector algorithm, whereby we first approximate the value of the field at the point in question (our point 1) using extrapolation, and then apply our finite-difference formula (3.21) iteratively, until the required accuracy is achieved. Specifically, we use the values of the perturbation $H$ at the four points 3, 6, 10, and 15 (see Fig. 3) to extrapolate the value $H_{1}$ with an error of $O\left(h^{4}\right)$. If the particle's worldline happens to cross the null segment 1-15 we instead use the points $2,5,7$, and 11 for this extrapolation. We then use the value of $H_{1}$ thus obtained as input in Eq. (3.21). Since the extrapolated terms enter Eq. (3.21) multiplied by at least one power of $h$ [see, e.g., Eqs. (3.6) and (3.7)], the resulting value of $H_{1}$ would have a local error of $O\left(h^{5}\right)$. We then apply Eq. (3.21) once more, with the new value of $H_{1}$ as input. The output value of $H_{1}$ following this second iteration should now have a local error of $O\left(h^{6}\right)$ as desired.

The above finite-difference scheme is designed to yield a local error of $O\left(h^{6}\right)$ in $\bar{h}_{1}^{(i)}$ at each grid point. Since the overall number of grid points contributing to the accumulated error scales as $\sim h^{-2}$, we expect our scheme to show a quartic [i.e., $\left.O\left(h^{4}\right)\right]$ numerical convergence.

\section{Monopole and dipole modes}

In the monopole case $(l=0)$ the system of 10 field equations (2.20) reduces to 4 equations only; it reduces to 6 equations for each of the two dipole modes $(l, m)=(1, \pm 1)$ and to a single equation for the dipole mode $(l, m)=(1,0)$. One may attempt to apply the above numerical evolution scheme to these non-radiative modes as well. However, numerical experimentation suggests to us that the monopole and dipole cannot be evolved stably using this scheme. A naive application of the evolution scheme yields exponentially growing solutions, and, since our scheme gives us no handle on the boundary conditions, the occurrence of these unphysical solutions is difficult to control.

Instead, we deal with the two modes $l=0,1$ using the standard frequency-domain method, just as in Paper I. The physical Lorenz-gauge monolpole and dipole are constructed from a basis of homogeneous frequency-mode solutions of the underlying ordinary differential equations. These homogeneous solutions are obtained numerically. However, unlike in the circular-orbit case dealt with in Paper I, here we face the "Gibbs phenomenon", since for an eccentric orbit the perturbation is a non-differentiable function of coordinate time $t$ at the particle's location. We have discussed this problem in depth in Ref. [31], and proposed an elegant solution, whereby the correct physical perturbation is constructed as a sum of "fake" frequency-mode solutions whose Fourier sum converges exponentially even at the particle's position. Here we apply this method in order to obtain the physical Lorenz-gauge monopole and dipole modes. A full description of this construction will be given in a forthcoming paper [43].

\section{Implementation of the mode-sum scheme}

Once we obtain the numerical values of the functions $\bar{h}^{(i)}$ and their derivatives along the orbit (over a complete radial period), we can construct the full-force modes $F_{\text {full } \pm}^{\alpha l}$ at any point along the orbit through the procedure described in Sec. IID The mode-sum formula (2.11) then gives the physical SF at that point. The application of the mode-sum formula involves summation over contributions from an infinite number of modes, from $l=0$ to $l=\infty$. In reality, of course, we are only able to compute a small number of low multipole modes - not least because the numerical calculation becomes increasingly more demanding with larger $l$. Since the mode-sum scheme converges rather slowly (as $\sim 1 / l$ ), a calculation of the $\mathrm{SF}$ with even a modest accuracy requires that we take a proper account of the contribution from the truncated tail of the mode sum.

Let us denote by $\bar{l}$ the highest spherical-harmonic $l$-mode calculated numerically (note this would entail calculating 
all tensor-harmonic modes from $l=0$ to $l=\bar{l}+3$ ). Recalling the notation of Eq. (2.11), we express the SF as

$$
F^{\alpha}=\sum_{l=0}^{\bar{l}} F_{\mathrm{reg}}^{\alpha l}+\sum_{l=\bar{l}+1}^{\infty} F_{\mathrm{reg}}^{\alpha l} \equiv F_{l \leq \bar{l}}^{\alpha}+F_{l>\bar{l}}^{\alpha},
$$

where $F_{l<\bar{l}}^{\alpha}$ is the part calculated numerically, and $F_{l>\bar{l}}^{\alpha}$ is the truncated tail we need to estimate. Here it is beneficial to consider separately the conservative and dissipative pieces of the SF. We remind that these can be constructed individually using the mode-sum formulas (2.29) and (2.30) via the procedure described in Sec. IIE

Consider the conservative piece first. The regularized force modes in this case admit the large- $l$ expansion

$$
F_{\text {reg(cons) }}^{\alpha l}=D_{-2}^{\alpha} L^{-2}+D_{-4}^{\alpha} L^{-4}+\ldots,
$$

where $D_{-2 n}^{\alpha}$ are $l$-independent coefficients. An approximation for these coefficients can be obtained by fitting a large- $l$ subset of numerical data to Eq. (3.23). In practice we take $\bar{l}=15$ and find the two coefficients $D_{-2}^{\alpha}$ and $D_{-4}^{\alpha}$ using the numerically-derived modes $10 \leq l \leq 15$. The large- $l$ tail piece of $F_{\text {reg(cons) }}^{\alpha l}$ is then approximated as

$$
F_{\text {cons }, l>\bar{l}}^{\alpha} \equiv \sum_{l=\bar{l}+1}^{\infty} F_{\text {reg(cons })}^{\alpha l} \cong D_{-2}^{\alpha} \Gamma_{1}(\bar{l}+3 / 2)+D_{-4}^{\alpha} \Gamma_{3}(\bar{l}+3 / 2) / 3 !,
$$

where $\Gamma_{n}(x)$ is the polygamma function of order $n$, defined in terms of the derivatives of the standard gamma function as $\Gamma_{n}(x)=d^{n+1}[\log \Gamma(x)] / d x^{n+1}$. Since the leading term omitted in Eq. (3.23) is of $O\left(L^{-6}\right)$, we expect the absolute error in our estimation of $F_{\text {cons }, l>\bar{l}}^{\alpha}$ to be of $O\left(\bar{l}^{-5}\right)$, or $O\left(\bar{l}^{-4}\right)$ fractionally. With $\bar{l}=15$ this amounts to a $\sim 10^{-5}$ fractional error, which we can afford to tolerate in this work.

Now consider the dissipative piece. We have that the magnitude of $F_{\text {reg(diss })}^{\alpha l}$ falls off faster than any power of $1 / l$ at large $l$. For the range of orbital parameters explored in this work we find that the numerical value of $F_{\text {reg(diss })}^{\alpha l}$ drops below the round-off error at $l \sim 7-12$, and so the large- $l$ tail, estimated as $F_{\text {diss }, l>\bar{l}}^{\alpha} \sim F_{\text {reg(diss) }}^{\alpha \bar{l}}$, can be safely neglected taking $\bar{l}=12$. In practice, to avoid adding up spurious round-off contributions, we truncate the mode-sum series at $\bar{l}=\min \{\hat{l}, 15\}$, where $\hat{l}$ is the first value of $l$ above $l=7$ for which we find $\left|F_{\text {reg(diss })}^{\alpha \hat{l}}\right|>\left|F_{\text {reg(diss) }}^{\alpha, \hat{l}-1}\right|$.

Our procedure for estimating the numerical error is as follows. First, we estimate the discretization error in each of the computed $l$-mode contributions (as a time series along the orbit) by repeating our numerical evolution at a coarser resolution and using the difference between the high-and low-resolution data sets as a crude error estimator. [For example, to obtain the data in Tables IIV] below we applied a cell size of $\Delta u \times \Delta v=(0.02 M)^{2}$ for our highest resolution runs, then $\Delta u \times \Delta v=(0.04 M)^{2}$ for error estimation.] The total error in the numerically-computed part of the SF is then taken (conservatively) as the sum of the errors (in absolute value) from the various modes. In the case of the conservative piece we add to this the estimated standard fitting error for the large- $l$ tail. For the fitting itself we use the high-resolution $l$-mode data points, weighted by their estimated discretization errors. This procedure yields a conservative estimate for the numerical error in each of $F_{\text {cons }}^{\alpha}(\chi)$ and $F_{\text {diss }}^{\alpha}(\chi)$. It is this error that we quote in the next section when presenting our results.

\section{E. Code validation and performance}

Using a few test runs with a range of sample parameters, we tested our code (i) for 4th-order numerical convergence; (ii) against the SF results of Paper I in the circular-orbit case $(e=0)$; (iii) by extracting the flux of energy and angular momentum carried by the gravitational waves and comparing with results in the literature (see Sec. IVA); and (iv) by verifying that the dissipative component of the computed SF precisely balances the above flux (Sec. IVA). Our code seems to perform well, at a standard fractional accuracy of $<10^{-4}$ in the final SF, across the parameter range $0 \leq e \lesssim 0.5$ and $p \lesssim 20 M$. For larger eccentricities and/or larger values of $p$ the long evolution time required begins to play a prohibitive role. Our code is still fully functional at (say) $(p, e)=(50,0.9)$, although in this case it becomes computationally impractical to achieve our standard $10^{-4}$ accuracy running on a standard (single) desktop computer.

In developing and testing our code we used a desktop workstation with a $2.5 \mathrm{GHz} 64$-bit processor and $8 \mathrm{~Gb}$ of RAM. A typical computation of the SF over a complete radial period, with given parameters in the above "workable" range (and at the above accuracy standard), demands 4-8 days of CPU time on this machine. 


\section{SAMPLE RESULTS}

Figure [6 displays SF results for the sample parameters $(p, e)=(10,0.2),(10,0.5)$ and $(10,0.7)$. We plot the temporal and radial components of the (total) SF along the geodesic orbit as functions of coordinate time $t$. The third non-trivial component, $F^{\varphi}$, can be obtained using the orthogonality condition $F^{\alpha} u_{\alpha}=0$. In these plots $t=0$ corresponds to a periapsis passage (where $r=r_{\min }$ ), so that, in accordance with the discussion at the end of Sec. IIE, the dissipative/conservative pieces of $F^{t}$ are described by the symmetric/anti-symmetric parts of the $F^{t}$-graph, and conversely for $F^{r}$.
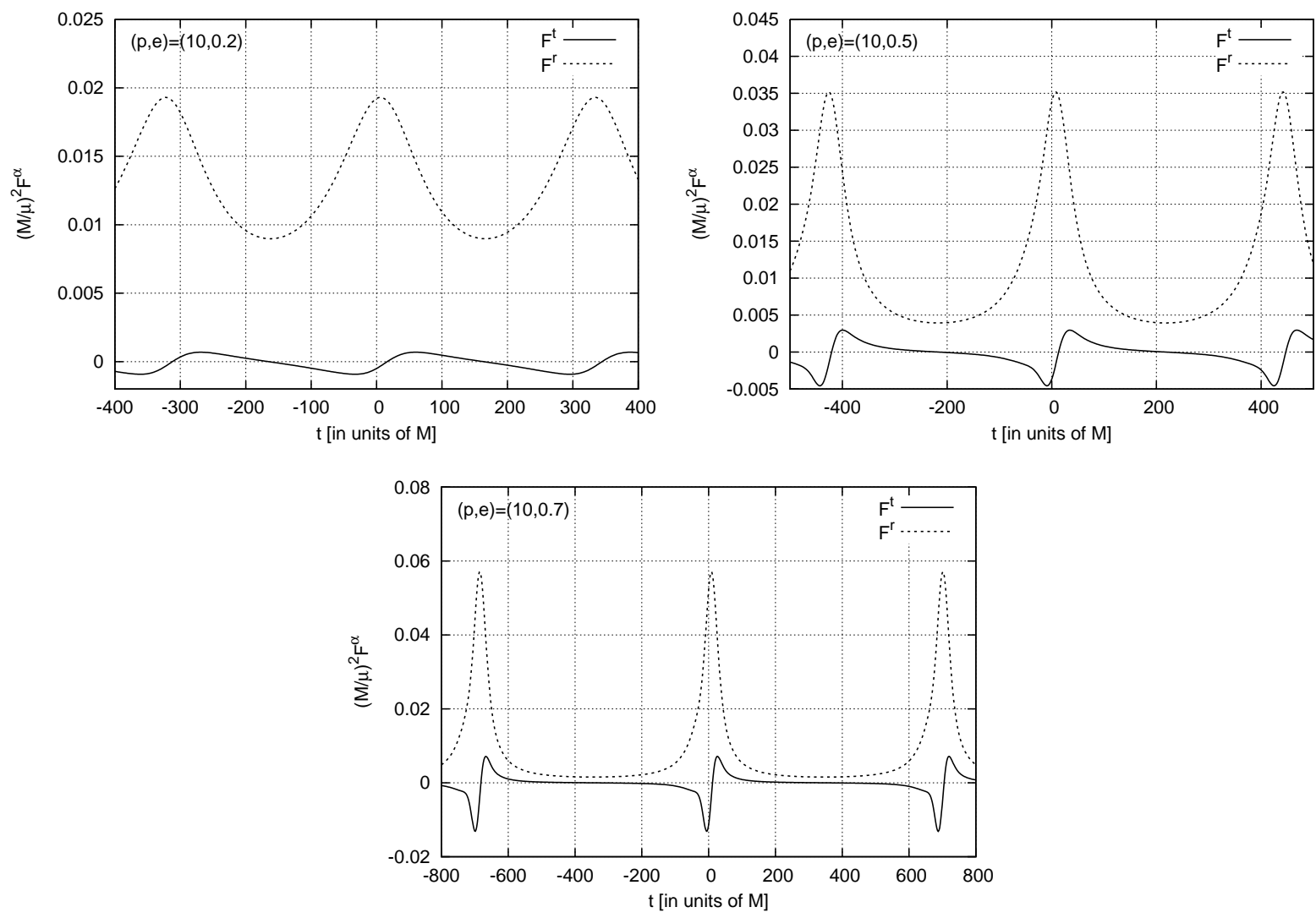

FIG. 6: The gravitational SF for a sample of eccentric orbits. We plot the temporal and radial components of the SF as functions of $t$ for the sample parameters $(p, e)=(10,0.2),(10,0.5)$ and $(10,0.7)$. The solid and dashed lines show $F^{t}$ and $F^{r}$, respectively. The $\varphi$ component of the SF can be trivially obtained using the orthogonality condition $F^{\alpha} u_{\alpha}=0$. In all graphs $t=0$ corresponds to a periapsis passage. The radial periods for the $e=0.2,0.5,0.7$ plots are, respectively, $T_{r} \simeq 328 M, 434 M, 693 M$. We have cut out from these plots the early part of the numerical solution, where non-physical initial spurious waves dominate; the plots display only the later, stationary part of the solutions, after the spurious waves have dissipated away. Note the small retardation manifest in the amplitude of the total SF (with reference to the orbital phase). A similar retardation is observed in the scalar and EM cases [12, 23].

In Tables IIV we present numerical SF results for the sets of orbital parameters $(p, e)=(7,0.2),(7,0.4),(10,0.3)$ and $(15,0.3)$, for a few sample values of the radial phase $\chi$. These numerical values can be used as a reference for testing future calculations of the SF. In the tables we display the conservative and dissipative pieces of the SF separately. We only display values for $F^{t}$ and $F^{r}$-once again, the azimuthal component can be obtained trivially using $F^{\alpha} u_{\alpha}=0$. Also, we only give values for the "outbound" half of the orbital period $\left(0 \leq \chi \leq \pi\right.$, where $\left.\dot{r}_{\mathrm{p}} \geq 0\right)$; the values for the "inbound" half $(\pi \leq \chi \leq 2 \pi)$ can be obtained immediately based on the symmetry relations (2.33). 


\begin{tabular}{c||c|c||c|c}
\hline \hline$\chi$ & $\eta^{-2} F_{\text {cons }}^{t}$ & $\eta^{-2} F_{\text {diss }}^{t}$ & $\eta^{-2} F_{\text {cons }}^{r}$ & $\eta^{-2} F_{\text {diss }}^{r}$ \\
\hline 0 & 0 & $-4.06330(3) \times 10^{-3}$ & $3.35760(5) \times 10^{-2}$ & 0 \\
$\pi / 8$ & $5.3846(3) \times 10^{-4}$ & $-3.47962(2) \times 10^{-3}$ & $3.23228(4) \times 10^{-2}$ & $3.148027(5) \times 10^{-3}$ \\
$\pi / 4$ & $8.6422(6) \times 10^{-4}$ & $-2.15692(2) \times 10^{-3}$ & $2.90989(5) \times 10^{-2}$ & $4.73496(1) \times 10^{-3}$ \\
$3 \pi / 8$ & $9.2840(3) \times 10^{-4}$ & $-9.2831(1) \times 10^{-4}$ & $2.50709(3) \times 10^{-2}$ & $4.47010(1) \times 10^{-3}$ \\
$\pi / 2$ & $8.2846(4) \times 10^{-4}$ & $-2.5168(3) \times 10^{-4}$ & $2.12504(2) \times 10^{-2}$ & $3.204188(5) \times 10^{-3}$ \\
$5 \pi / 8$ & $6.61185(8) \times 10^{-4}$ & $-3.385(1) \times 10^{-5}$ & $1.81454(2) \times 10^{-2}$ & $1.893665(3) \times 10^{-3}$ \\
$3 \pi / 4$ & $4.60708(2) \times 10^{-4}$ & $-1.1241(3) \times 10^{-5}$ & $1.590157(5) \times 10^{-2}$ & $9.63374(2) \times 10^{-4}$ \\
$7 \pi / 8$ & $2.36409(2) \times 10^{-4}$ & $-2.7138(1) \times 10^{-5}$ & $1.454424(7) \times 10^{-2}$ & $3.90516(1) \times 10^{-4}$ \\
$\pi$ & 0 & $-3.4614(2) \times 10^{-5}$ & $1.40888(2) \times 10^{-2}$ & 0 \\
\hline \hline
\end{tabular}

TABLE I: The gravitational SF at selected points along an eccentric geodesic with $(p, e)=(7,0.2) . \eta \equiv \mu / M$. In the first column $\chi$ is the radial phase along the orbit [cf. Eq. [2.5] ], with $\chi=0, \pi$ corresponding to periapsis and apoaspsis, respectively. Subsequent columns display, in order, the conservative and dissipative pieces of $F^{t}$ and the conservative and dissipative pieces of $F^{r}$. Values in brackets are estimates of the uncertainty (due to numerical error) in the last displayed decimal place. For example, $5.3844(3) \times 10^{-4}$ stands for $(5.3844 \pm 0.0003) \times 10^{-4}$. The values for $F^{\varphi}$ can be obtained from $F^{\alpha} u_{\alpha}=0$. Values for the SF along the "inbound" half of the radial period $(\pi \leq \chi \leq 2 \pi)$ can be deduced based on the symmetry relations (2.33).

\begin{tabular}{c||c|c|c|c}
\hline \hline$\chi$ & $\eta^{-2} F_{\text {cons }}^{t}$ & $\eta^{-2} F_{\text {diss }}^{t}$ & $\eta^{-2} F_{\text {cons }}^{r}$ & $\eta^{-2} F_{\text {diss }}^{r}$ \\
\hline 0 & 0 & $-1.509113(8) \times 10^{-2}$ & $5.56081(6) \times 10^{-2}$ & 0 \\
$\pi / 8$ & $1.8006(1) \times 10^{-3}$ & $-1.142319(3) \times 10^{-2}$ & $5.05974(8) \times 10^{-2}$ & $1.828802(3) \times 10^{-2}$ \\
$\pi / 4$ & $2.5896(2) \times 10^{-3}$ & $-3.67901(9) \times 10^{-3}$ & $3.90832(9) \times 10^{-2}$ & $2.28485(2) \times 10^{-2}$ \\
$3 \pi / 8$ & $1.9915(2) \times 10^{-3}$ & $1.26976(5) \times 10^{-3}$ & $2.77839(3) \times 10^{-2}$ & $1.605261(1) \times 10^{-2}$ \\
$\pi / 2$ & $1.30621(8) \times 10^{-3}$ & $1.74587(2) \times 10^{-3}$ & $1.98195(4) \times 10^{-2}$ & $7.59798(5) \times 10^{-3}$ \\
$5 \pi / 8$ & $9.8662(4) \times 10^{-4}$ & $8.03401(6) \times 10^{-4}$ & $1.47071(2) \times 10^{-2}$ & $2.65711(2) \times 10^{-3}$ \\
$3 \pi / 4$ & $7.0011(1) \times 10^{-4}$ & $2.396343(8) \times 10^{-4}$ & $1.13351(1) \times 10^{-2}$ & $7.76246(5) \times 10^{-4}$ \\
$7 \pi / 8$ & $3.49621(1) \times 10^{-4}$ & $6.31089(3) \times 10^{-5}$ & $9.28115(1) \times 10^{-3}$ & $2.04288(2) \times 10^{-4}$ \\
$\pi$ & 0 & $2.92505(1) \times 10^{-5}$ & $8.581533(5) \times 10^{-3}$ & 0 \\
\hline \hline
\end{tabular}

TABLE II: The gravitational SF at selected points along an eccentric geodesic with $(p, e)=(7,0.4)$. The structure of the table is the same as that of Table. I

\section{A. Dissipation of energy and angular momentum}

Given the local SF, we can calculate the (orbit-averaged) rate at which orbital energy and angular momentum are dissipated. This information is contained in the $t$ and $\varphi$ components of the local SF. From Eq. (2.10) one readily obtains

$$
\dot{\mathcal{E}}(\chi)=-\left[\mu u^{t}(\chi)\right]^{-1} F_{t}(\chi), \quad \dot{\mathcal{L}}(\chi)=\left[\mu u^{t}(\chi)\right]^{-1} F_{\varphi}(\chi)
$$

\begin{tabular}{c||c|c|c|c}
\hline \hline$\chi$ & $\eta^{-2} F_{\text {cons }}^{t}$ & $\eta^{-2} F_{\text {diss }}^{t}$ & $\eta^{-2} F_{\text {cons }}^{r}$ & $\eta^{-2} F_{\text {diss }}^{r}$ \\
\hline 0 & 0 & $-1.024249(7) \times 10^{-3}$ & $2.303169(9) \times 10^{-2}$ & 0 \\
$\pi / 8$ & $7.2278(2) \times 10^{-4}$ & $-8.05046(5) \times 10^{-4}$ & $2.21659(3) \times 10^{-2}$ & $8.51208(8) \times 10^{-4}$ \\
$\pi / 4$ & $1.16148(2) \times 10^{-3}$ & $-3.67855(4) \times 10^{-4}$ & $1.98540(2) \times 10^{-2}$ & $1.17785(3) \times 10^{-3}$ \\
$3 \pi / 8$ & $1.247664(9) \times 10^{-3}$ & $-6.1078(3) \times 10^{-5}$ & $1.677294(8) \times 10^{-2}$ & $9.63601(6) \times 10^{-4}$ \\
$\pi / 2$ & $1.08725(1) \times 10^{-3}$ & $3.3434(2) \times 10^{-5}$ & $1.36220(1) \times 10^{-2}$ & $5.65458(5) \times 10^{-4}$ \\
$5 \pi / 8$ & $8.11160(7) \times 10^{-4}$ & $2.83103(3) \times 10^{-5}$ & $1.088087(7) \times 10^{-2}$ & $2.63807(5) \times 10^{-4}$ \\
$3 \pi / 4$ & $5.122807(9) \times 10^{-4}$ & $1.10418(3) \times 10^{-5}$ & $8.81008(2) \times 10^{-3}$ & $1.06375(1) \times 10^{-4}$ \\
$7 \pi / 8$ & $2.408105(6) \times 10^{-4}$ & $2.45312(6) \times 10^{-6}$ & $7.537692(9) \times 10^{-3}$ & $3.61963(8) \times 10^{-5}$ \\
$\pi$ & 0 & $2.836(2) \times 10^{-7}$ & $7.110909(3) \times 10^{-3}$ & 0 \\
\hline \hline
\end{tabular}

TABLE III: The gravitational SF at selected points along an eccentric geodesic with $(p, e)=(10,0.3)$. The structure of the table is the same as that of Table. [ 


\begin{tabular}{c||c|c|c|c}
\hline \hline$\chi$ & $\eta^{-2} F_{\text {cons }}^{t}$ & $\eta^{-2} F_{\text {diss }}^{t}$ & $\eta^{-2} F_{\text {cons }}^{r}$ & $\eta^{-2} F_{\text {diss }}^{r}$ \\
\hline 0 & 0 & $-1.040267(9) \times 10^{-4}$ & $1.139648(3) \times 10^{-2}$ & 0 \\
$\pi / 8$ & $3.03529(3) \times 10^{-4}$ & $-8.23882(8) \times 10^{-5}$ & $1.102460(1) \times 10^{-2}$ & $1.03215(6) \times 10^{-4}$ \\
$\pi / 4$ & $5.07593(2) \times 10^{-4}$ & $-3.9157(1) \times 10^{-5}$ & $1.000022(2) \times 10^{-2}$ & $1.43970(1) \times 10^{-4}$ \\
$3 \pi / 8$ & $5.68296(2) \times 10^{-4}$ & $-8.3320(6) \times 10^{-6}$ & $8.56001(2) \times 10^{-3}$ & $1.19734(3) \times 10^{-4}$ \\
$\pi / 2$ & $5.06147(1) \times 10^{-4}$ & $1.97928(7) \times 10^{-6}$ & $6.99810(1) \times 10^{-3}$ & $7.2183(3) \times 10^{-5}$ \\
$5 \pi / 8$ & $3.765543(7) \times 10^{-4}$ & $2.2286(2) \times 10^{-6}$ & $5.578997(8) \times 10^{-3}$ & $3.4786(1) \times 10^{-5}$ \\
$3 \pi / 4$ & $2.340696(1) \times 10^{-4}$ & $8.4719(9) \times 10^{-7}$ & $4.486884(2) \times 10^{-3}$ & $1.4418(1) \times 10^{-5}$ \\
$7 \pi / 8$ & $1.0842785(8) \times 10^{-4}$ & $1.0087(2) \times 10^{-7}$ & $3.814949(2) \times 10^{-3}$ & $4.9887(8) \times 10^{-6}$ \\
$\pi$ & 0 & $-9.092(5) \times 10^{-8}$ & $3.590240(2) \times 10^{-3}$ & 0 \\
\hline \hline
\end{tabular}

TABLE IV: The gravitational SF at selected points along an eccentric geodesic with $(p, e)=(15,0.3)$. The structure of the table is the same as that of Table. [1]

where in this section (unlike elsewhere in this work) an overdot denotes $d / d t$. We shall assume here, in effect, that $\mu / M$ is sufficiently small that the back-reaction effect on the orbit over a period of a few $T_{r}$ can be neglected. At this "adiabatic" limit the functions $\dot{\mathcal{E}}(\chi)$ and $\dot{\mathcal{L}}(\chi)$ are periodic in $t$ with period $T_{r}$, and their time-average is hence given by

$$
\langle\dot{\mathcal{E}}\rangle=\frac{1}{T_{r}} \int_{0}^{T_{r}} \dot{\mathcal{E}} d t, \quad\langle\dot{\mathcal{L}}\rangle=\frac{1}{T_{r}} \int_{0}^{T_{r}} \dot{\mathcal{L}} d t
$$

These averages determine the "secular" dissipative drift in the values of $\mathcal{E}$ and $\mathcal{L}$. Note that $\langle\dot{\mathcal{E}}\rangle$ and $\langle\dot{\mathcal{L}}\rangle$ depend only on the dissipative components $F_{\text {diss }}^{t}$ and $F_{\text {diss }}^{\varphi}$ (respectively); it is immediately evident from the symmetry relations (2.33) that the contributions from $F_{\text {cons }}^{t}$ and $F_{\text {cons }}^{\varphi}$ vanish upon orbital-averaging. We also note that the dissipative radial component $F_{\text {diss }}^{r}$ (let alone $F_{\text {cons }}^{r}$ ) has no effect on the values of $\langle\dot{\mathcal{E}}\rangle$ and $\langle\dot{\mathcal{L}}\rangle$. The secular drifts $\langle\dot{\mathcal{E}}\rangle$ and $\langle\dot{\mathcal{L}}\rangle$ must be balanced by the flux of energy and azimuthal angular momentum in the gravitational waves radiated to infinity and down the event horizon. Denoting the respective energy fluxes by $\langle\dot{E}\rangle_{\infty} / \mathrm{EH}$ and angular momentum fluxes by $\langle\dot{L}\rangle_{\infty / \mathrm{EH}}$, we have the balance equations

$$
\begin{aligned}
& -\mu\langle\dot{\mathcal{E}}\rangle=\langle\dot{E}\rangle_{\infty}+\langle\dot{E}\rangle_{\mathrm{BH}} \equiv\langle\dot{E}\rangle_{\text {total }}, \\
& -\mu\langle\dot{\mathcal{L}}\rangle=\langle\dot{L}\rangle_{\infty}+\langle\dot{L}\rangle_{\mathrm{BH}} \equiv\langle\dot{L}\rangle_{\text {total }} .
\end{aligned}
$$

Two validation tests for our code now suggest themselves. First, we may attempt to extract the asymptotic fluxes directly from our numerically-calculated Lorenz-gauge metric perturbation, and compare with results in the literature. Second, using Eqs. (4.1) we can derive the local quantities $\langle\dot{\mathcal{E}}\rangle$ and $\langle\dot{\mathcal{L}}\rangle$ from our SF results, and check whether the balance equations (4.3) and (4.4) are indeed satisfied.

For both above tests we need a time-domain formulation of the asymptotic fluxes in Schwarzschild spacetime. Such a formulation was presented by Martel [44] and Poisson [45] [64], and we shall adopt it here. Martel-Poisson's construction is based on the Regge-Wheeler and Zerilli-Moncrief perturbations functions $\Psi_{\mathrm{RW}}^{l m}$ and $\Psi_{\mathrm{ZM}}^{l m}$, which are related to our Lorenz-gauge variables through

$$
\begin{aligned}
\Psi_{\mathrm{RW}}^{l m} & =-\frac{(l-2) !}{2(l+2) !}\left[\frac{\lambda}{r} \bar{h}^{(9)}+\frac{f}{r} \bar{h}^{(10)}-\bar{h}_{, r_{*}}^{(10)}\right], \\
\Psi_{\mathrm{ZM}}^{l m} & =\frac{2 r}{l(l+1)(\lambda r+6 M)}\left[\bar{h}^{(1)}-\bar{h}^{(5)}-f \bar{h}^{(6)}+\frac{l(l+1) r+2 M}{2 r} \bar{h}^{(3)}-r \bar{h}_{, r_{*}}^{(3)}+\frac{\lambda r+6 M}{2 \lambda r} \bar{h}^{(7)}\right],
\end{aligned}
$$

with $\lambda \equiv(l+2)(l-1)$. In terms of $\Psi_{\mathrm{RW}}^{l m}$ and $\Psi_{\mathrm{ZM}}^{l m}$, the fluxes at infinity are given by [44, 45]

$$
\begin{aligned}
\langle\dot{E}\rangle_{\infty} & =\frac{1}{64 \pi} \sum_{l m} \frac{(l+2) !}{(l-2) !}\left\langle 4\left|\Psi_{\mathrm{RW}}^{l m}(u)\right|^{2}+\left|\dot{\Psi}_{\mathrm{ZM}}^{l m}(u)\right|^{2}\right\rangle, \\
\langle\dot{L}\rangle_{\infty} & =\frac{1}{64 \pi} \sum_{l m} \frac{(l+2) !}{(l-2) !}(i m)\left\langle 4 \Psi_{\mathrm{RW}}^{l m}(u) \int^{u} \Psi_{\mathrm{RW}}^{l m *}\left(u^{\prime}\right) d u^{\prime}+\dot{\Psi}_{\mathrm{ZM}}^{l m}(u) \Psi_{\mathrm{ZM}}^{l m *}(u)\right\rangle,
\end{aligned}
$$

where an asterisk denotes complex conjugation, $\langle\cdots\rangle$ indicates a suitable time-average (in our case an average over a period $T_{r}$ would suffice), and the functions $\Psi_{\mathrm{RW}}^{l m}$ and $\Psi_{\mathrm{ZM}}^{l m}$ are evaluated at the "wave zone", $v \rightarrow \infty$ with fixed $u$. 


\begin{tabular}{lccc}
\hline \hline & This paper (TD) & Martel (TD) & Fujita et al. (FD) \\
\hline$p=7.50478, e=0.188917$ & & & \\
$\langle\dot{E}\rangle_{\infty} \times 10^{4} \eta^{-2}$ & $3.169(1)$ & 3.1770 & 3.16899989184 \\
$\langle\dot{L}\rangle_{\infty} \times 10^{3} \eta^{-2} M^{-1}$ & $5.96760(8)$ & 5.9329 & 5.96755215608 \\
\hline$p=8.75455, e=0.764124$ & & & \\
$\langle\dot{E}\rangle_{\infty} \times 10^{4} \eta^{-2}$ & $2.124(3)$ & 2.1484 & 2.12360313326 \\
$\langle\dot{L}\rangle_{\infty} \times 10^{3} \eta^{-2} M^{-1}$ & $2.7774(6)$ & 2.7932 & 2.77735938996 \\
\hline \hline
\end{tabular}

TABLE V: The flux of energy and angular momentum in the gravitational waves radiated to infinity: comparison with results in the literature. The second column shows the values of the radiative fluxes $\langle\dot{E}\rangle_{\infty}$ and $\langle\dot{L}\rangle_{\infty}$, evaluated from our numerical results using Eq. (4.7) and (4.8) for two sample values of $p, e$. Values in parentheses estimate the uncertainty in the last displayed figure. The subsequent columns display, for comparison, the corresponding values obtained by Martel [44] and Fujita et al. [47]. TD/FD indicate time/frequency-domain methods. Fujita et al. claim all their displayed figures are significant.

The horizon fluxes $\langle\dot{E}\rangle_{\mathrm{BH}}$ and $\langle\dot{L}\rangle_{\mathrm{BH}}$ are given by expressions similar to (4.7) and (4.8), merely replacing $u \rightarrow v$ and evaluating $\Psi_{\mathrm{RW}}^{l m}$ and $\Psi_{\mathrm{ZM}}^{l m}$ near the horizon, i.e., at $u \rightarrow \infty$ with fixed $v$.

To calculate $\langle\dot{E}\rangle_{\infty}$ and $\langle\dot{L}\rangle_{\infty}$, we start by recording the numerical values of the perturbation functions $\bar{h}^{(i)}(u)$ at $v=10000 M$ (approximating null infinity), over a complete radial period. The desired fluxes at infinity are then calculated using Eqs. (4.5)-(4.8), making sure that the error from truncating the sum over $l$ is properly controlled (this is not difficult, as the mode sum converges exponentially; in none of the cases considered here we found it necessary to include modes beyond $l=12$ ). In a similar manner we also construct the horizon fluxes $\langle\dot{E}\rangle_{\mathrm{EH}}$ and $\langle\dot{L}\rangle_{\mathrm{EH}}$, starting by extracting the numerical values $\bar{h}^{(i)}(v)$ at very large $u$ (approximating the horizon; in practice we used $u=10000 M$ ), and then using Eq. (4.5) and (4.6) with the horizon-version of Eqs. (4.7) and (4.8). We estimate the error in our flux values by comparing results obtained at two different grid resolutions $(h=0.1$ against $h=0.2)$.

The eccentric-orbit fluxes $\langle\dot{E}\rangle_{\infty}$ and $\langle\dot{L}\rangle_{\infty}$ were computed independently in the past by several authors, including Tanaka et al. [46] and Cutler et al. [38] [using frequency-domain (FD) analyses based on Teukolsky's formalism], and later by Martel 44] [using a time-domain (TD) analysis based on the Regge-Wheeler-Zerilli formalism]. Very recently, Fujita et al. [47] developed a highly accurate FD algorithm for flux calculations. In Table $\mathrm{V}$ we compare our flux data with the TD data of Martel and the FD data of Fujita et al. [48]. We look at two strong-field orbits, one with moderate eccentricity $(e \simeq 0.19)$ and the other with a rather high eccentricity $(e \simeq 0.76)$. All the results shown agree with ours to within $1 \%$. The FD results agree with ours to within at least $0.01 \%$, and in all cases the FD results fall well within our estimated error bars. Martel's TD results were presented without error bars, but they are likely less accurate than the FD ones.

In Tables VI and VII we carry out the second test mentioned above, i.e., we check whether our numerical SF data and flux data satisfy the balance equations (4.3) and (4.4). The tables compare the time-averaged rates of loss of orbital energy and angular momentum, $\mu\langle\dot{\mathcal{E}}\rangle$ and $\mu\langle\dot{\mathcal{L}}\rangle$, with the corresponding total (horizon+infinity) fluxes $\langle\dot{E}\rangle_{\text {total }}$ and $\langle\dot{L}\rangle_{\text {total }}$. We find that the two are consistent with each other to within the numerical accuracy. This constitutes a significant, highly non-trivial validation test for our code.

Tables VI and VII also display the partial contributions to the fluxes $\langle\dot{E}\rangle_{\text {total }}$ and $\langle\dot{L}\rangle_{\text {total }}$ coming form black hole absorption, i.e., $\langle\dot{E}\rangle_{\mathrm{BH}}$ and $\langle\dot{L}\rangle_{\mathrm{BH}}$. Note that our numerical accuracy is sufficient to confidently resolve these (relatively minute) horizon fluxes in the examples considered. The consistency of the local dissipative SF with the asymptotic fluxes, at the accuracy level maintained here, is evident only when black hole absorption is correctly accounted for. The data in Tables VI and VII represent a first numerical test of the Martel-Poisson TD horizon-flux formula.

\section{B. Zoom-whirl orbits}

An interesting family of eccentric geodesics, so called "zoom-whirl" orbits [49], has $p-6-2 e \equiv \epsilon \ll 1$. These geodesics correspond to points in the $p, e$ plane lying very close to the separatrix (see Fig. 1), possessing energy-squared $\mathcal{E}^{2}$ only slightly smaller than the maximum of the effective potential $R\left(r, \mathcal{L}^{2}\right)$. A particle on a zoom-whirl orbit spends most of the radial period "whirling" around the central hole in a nearly circular orbit near periapsis, before "zooming out" back to apoapsis distance. During the whirl episode the particle may complete many revolutions in $\varphi$ - the $\varphi$-phase accumulated over one radial period scales as $\Delta \varphi \propto \ln (64 e / \epsilon)$ [see Eq. (2.25) in [38]].

In Fig. 7 we show the SF along a sample zoom-whirl orbit with parameters $(p, e)=(7.4001,0.7)$. All components of the SF are relatively very small near the apoapsis, as would be expected in virtue of the large distance from the central black hole. During the brief "zoom-in" and "zoom out" episodes the particle has a large radial velocity component, 


\begin{tabular}{c|c||c|c|c|c}
\hline \hline$p$ & $e$ & $-\mu\langle\dot{\mathcal{E}}\rangle \times 10^{4} \eta^{-2}$ & $\langle\dot{E}\rangle_{\text {total }} \times 10^{4} \eta^{-2}$ & $\langle\dot{E}\rangle_{\mathrm{BH}} /\langle\dot{E}\rangle_{\text {total }}$ & $1+\mu\langle\dot{\mathcal{E}}\rangle /\langle\dot{E}\rangle_{\text {total }}$ \\
\hline 7.0 & 0.0 & $4.00163(5)$ & $4.00166(4)$ & $1.3 \times 10^{-3}$ & $7 \times 10^{-6}$ \\
7.0 & 0.1 & $4.21696(9)$ & $4.217(2)$ & $1.6 \times 10^{-3}$ & $9 \times 10^{-6}$ \\
7.0 & 0.2 & $4.8983(2)$ & $4.898(3)$ & $2.7 \times 10^{-3}$ & $-6 \times 10^{-5}$ \\
7.0 & 0.3 & $6.1852(3)$ & $6.185(4)$ & $4.6 \times 10^{-3}$ & $-3 \times 10^{-5}$ \\
7.0 & 0.4 & $8.5436(5)$ & $8.544(5)$ & $7.8 \times 10^{-3}$ & $5 \times 10^{-5}$ \\
\hline \hline
\end{tabular}

TABLE VI: Testing our code using the global energy balance equation (4.3). The third column displays the (negative of the) average rate of loss of orbital energy over one radial period, $-\mu\langle\dot{\mathcal{E}}\rangle$, for strong-field orbits with semi-latus rectum $p=7$ and a range of eccentricities $e$. This quantity is calculated from local SF data using Eqs. (4.1) and (4.2). For comparison, we give in the fourth column the corresponding values of the total energy fluxes $\langle\dot{E}\rangle_{\text {total }}$ in the gravitational waves radiated to infinity and down the event horizon. These fluxes are extracted from our numerically-derived Lorenz-gauge metric perturbation, evaluated at the corresponding asymptotic domains. Values in parentheses are estimates of the numerical error in the last displayed figure. The last column shows the relative difference between $-\mu\langle\dot{\mathcal{E}}\rangle$ and $\langle\dot{E}\rangle_{\text {total }}$, confirming that the balance equations are satisfied to within our working precision. For reference, the fifth column shows the relative contribution to $\langle\dot{E}\rangle_{\text {total }}$ from horizon absorption alone, denoted $\langle\dot{E}\rangle_{\mathrm{BH}}$. Manifestly, for the strong-field orbits considered here and with our working precision of $<10^{-4}$, black hole absorption cannot be neglected.

\begin{tabular}{c|c||c|c|c|c}
\hline \hline$p$ & $e$ & $-\langle\dot{\mathcal{L}}\rangle \times 10^{3} \eta^{-1}$ & $\langle\dot{L}\rangle_{\text {total }} \times 10^{3}\left(M / \mu^{2}\right)$ & $\langle\dot{L}\rangle_{\mathrm{BH}} /\langle\dot{L}\rangle_{\text {total }}$ & $1+\mu\langle\dot{\mathcal{L}}\rangle /\langle\dot{L}\rangle_{\text {total }}$ \\
\hline 7.0 & 0.0 & $7.41112(9)$ & $7.41124(7)$ & $1.3 \times 10^{-3}$ & $2 \times 10^{-5}$ \\
7.0 & 0.1 & $7.5896(2)$ & $7.58961(7)$ & $1.6 \times 10^{-3}$ & $1 \times 10^{-6}$ \\
7.0 & 0.2 & $8.1517(4)$ & $8.15137(7)$ & $2.3 \times 10^{-3}$ & $-4 \times 10^{-5}$ \\
7.0 & 0.3 & $9.2090(5)$ & $9.2092(2)$ & $4.0 \times 10^{-3}$ & $2 \times 10^{-5}$ \\
7.0 & 0.4 & $11.1627(7)$ & $11.164(2)$ & $7.0 \times 10^{-3}$ & $1 \times 10^{-4}$ \\
\hline \hline
\end{tabular}

TABLE VII: Testing our code using the global angular-momentum balance equation (4.4). The structure of this table is similar to that of Table VI. The table compares between the average rate of change of orbital angular momentum, $\langle\dot{\mathcal{L}}\rangle$, as inferred from local SF data, and the flux of angular momentum carried away by the gravitational waves, $\langle\dot{L}\rangle_{\text {total }}$, as inferred from the asymptotic waveforms. We also indicate the relative contribution to $\langle\dot{L}\rangle_{\text {total }}$ from angular momentum absorbed by the black hole, denoted $\langle\dot{L}\rangle_{\mathrm{BH}}$.

and the SF changes rapidly. During the whirl phase the particle moves on a nearly-circular orbit, and we expect the SF to settle to a constant value (this expectation is indeed confirmed in studies of the scalar-field and EM SFs $[12,23])$. We find that while this is true for the $t$ and $\varphi$ components, the $r$-component of the SF shows an unexpected linear-in- $t$ behavior during the whirl. Other zoom-whirl orbits we examined showed a similar behavior.

In order to understand the above peculiarity we conducted several experiments. The following points summarize the information gained. (i) The linearly growing piece of $F^{r}$ is entirely dissipative; the conservative piece $F_{\text {cons }}^{r}$ shows no such linear growth (see Fig. 8). (ii) The linearly growing piece of $F_{\text {diss }}^{r}$ is entirely attributed to the time-dependent piece of the monopole $(l=0)$ contribution to the SF; other modes show no such behavior. (iii) When examining a sequence of zoom-whirl orbits approaching the separatrix $(\epsilon \rightarrow 0)$ with fixed $e$ - see Fig. 8 - we observe that the rate of linear growth decreases with $\epsilon$, although rather slowly (slower than $\propto \epsilon$ ). It is possible that our numerical results are in fact consistent with $F_{\text {diss }}^{r} \rightarrow 0$ as $\epsilon \rightarrow 0$ (as expected), but this is difficult to verify numerically. (iv) We have applied our numerical algorithm to study the zoom-whirl behavior of the scalar-field SF. As in [12], we observed no linear behavior during the whirl. 65]

The last point (iv) serves to reaffirm our trust in the numerical code. The combination of points (i) and (ii) (together with the fact that no linear growth is observed in the scalar and EM cases) implies that the linear behavior is purely gauge-related, and suggests that it should have no observational (gauge invariant) consequences. In particular, the culprit monopole piece of $F_{\text {diss }}^{r}$ is non-radiative and has no secular physical effect on the orbit. Finally, the observation made in (iii) suggests there is nothing wrong with our choice of gauge either. In fact, it may be argued that the observed linear-in- $t$ behavior, with a weak dependence on $\epsilon$, is perfectly consistent with the theoretical expectation based on a local analysis of $F_{\text {diss }}^{r}$ near the separatrix. We explain this in the following.

Consider the behavior of $F_{\text {diss }}^{r}$ for a zoom-whirl orbit, $\epsilon \ll 1$. During the whirl the radial phase $\chi$ changes very little, so, taking $\chi=0$ at periapsis as usual, we can assume $\chi \ll 1$. In the following analysis we fix the eccentricity $e(>0)$ and consider the limit $\epsilon \rightarrow 0$ by taking $p \rightarrow 6+2 e$ (from above), inspecting the behavior of $F_{\text {diss }}^{r}$ at leading order in both $\chi$ and $\epsilon$. A convenient starting point for this analysis is the orthogonality condition $u_{\alpha} F^{\alpha}=0$, whose 


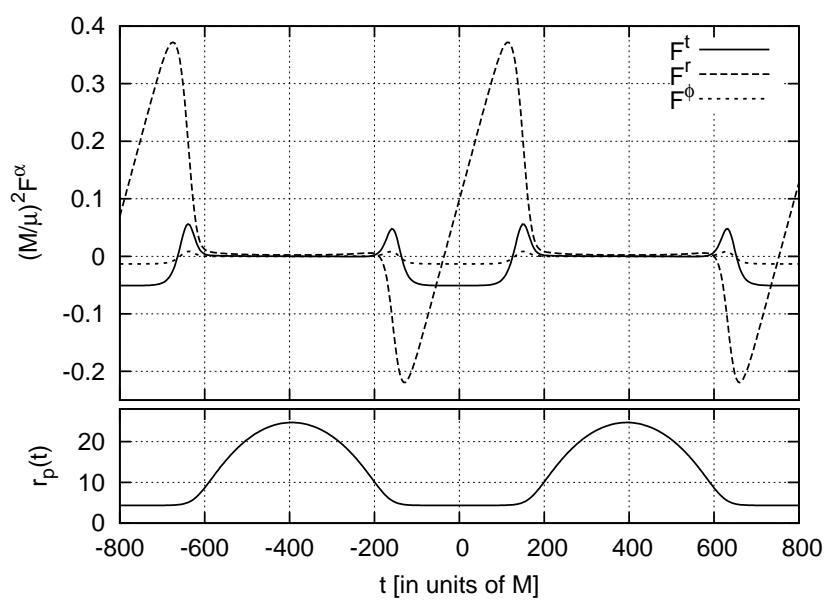

FIG. 7: The gravitational SF for a zoom-whirl orbit with parameters $(p, e)=(7.4001,0.7)$. In the upper panel we show the (contravariant) $t, r$, and $\varphi$ components of the SF as functions of time $t$ along the orbit. The lower panel shows the radial motion of the particle, for reference. $t=0$ is periapsis, and the radial period is $\sim 789 M$. While $F^{t}$ and $F^{\varphi}$ quickly settle to a constant value during the quasi-circular whirl episode (as one would expect), the radial component exhibits a peculiar linear behavior. This behavior is analyzed in the text (cf. Fig. 8).
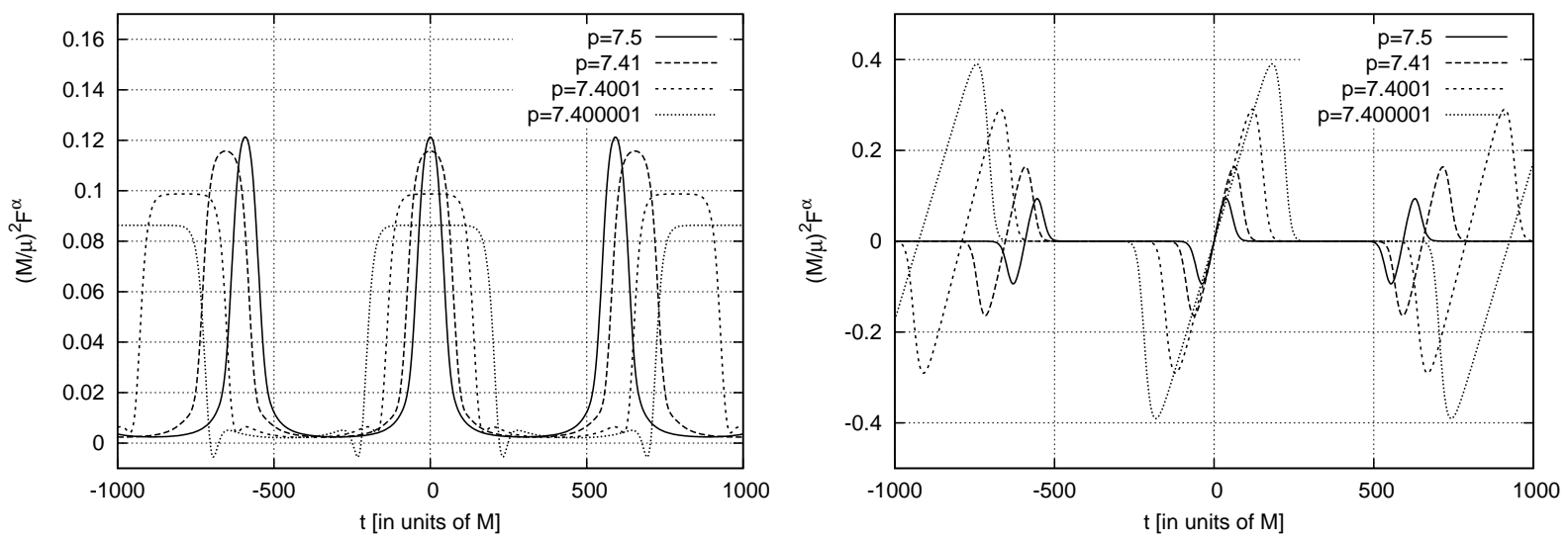

FIG. 8: Conservative and dissipative pieces of the radial SF for zoom-whirl orbits - left and right panels, respectively. Shown are results for four orbits with the same eccentricity $(e=0.7)$ but with increasing proximity to the separatrix $p=6+2 e$.

dissipative part can be rearranged to give

$$
\mu^{-1} F_{\text {diss }}^{r}=\frac{\left(\mathcal{E}^{2} / f_{\mathrm{p}}\right)\left(\dot{\mathcal{E}}_{\text {diss }}-\Omega \dot{\mathcal{L}}_{\text {diss }}\right)}{u^{r}} \equiv \frac{\left(\mathcal{E}^{2} / f_{\mathrm{p}}\right) \Psi}{u^{r}} .
$$

Here we have used the dissipative part of Eqs. (4.1), and denoted $\Omega \equiv d \varphi_{\mathrm{p}} / d t$. Recall $f_{\mathrm{p}}=1-2 M / r_{\mathrm{p}}$ and an overdot denotes $d / d t$. The factor $\left(\mathcal{E}^{2} / f_{\mathrm{p}}\right)$ is regular at $\chi=\epsilon=0$ and hence uninteresting. For the radial velocity, Eq. (2.8) gives $u^{r} \propto \epsilon^{1 / 2} \chi$, where throughout our present discussion a proportionately symbol implies the leading-order scaling with $\chi$ and $\epsilon$. The function $\Psi$ is symmetric in $\chi$ [recall Eq. [2.33)] and clearly $\Psi(\chi=0)=0$; hence we write $\Psi=\chi^{2} \psi(e, \epsilon)+O\left(\chi^{4}\right)$, where $\psi$ is $\chi$-independent. Taking now $t(\chi=0)=0$, Eq. (2.6) gives $\chi \propto \epsilon^{1 / 2} t$, and we thus obtain $u^{r} \propto \epsilon t$ and $\Psi \propto \epsilon t^{2} \psi(e, \epsilon)$. We conclude that

$$
F_{\text {diss }}^{r} \propto t \psi(e, \epsilon) .
$$

It is now essential to understand the scaling of $\psi$ with $\epsilon$ as $\epsilon \rightarrow 0$. Clearly, $\psi \rightarrow 0$ at this limit, since for circular orbits we have $\dot{\mathcal{E}}_{\text {diss }}=\Omega \dot{\mathcal{L}}_{\text {diss. }}$ It may be argued [38, 49$]$ that $\psi$ scales like the (small) fraction of the radial period that the particle is spending in the zoom phase, which, in turn, is proportional to $2 \pi / \Delta \varphi \propto[\ln (64 e / \epsilon)]^{-1}$. If this argument is to be trusted, we obtain

$$
F_{\text {diss }}^{r} \propto t \times[\ln (64 e / \epsilon)]^{-1},
$$


which may explain the very weak $\epsilon$-dependence of the slope in Fig. 8. It is difficult in practice to test our numerical results more quantitatively against the scaling relation (4.11) (precisely because the $\epsilon$-dependence is so weak), but we cannot rule out the possibility that our results are in fact consistent with this scaling.

It remains to understand why the linear mode does not exhibit itself so pronouncedly in the scalar and EM cases, and how the amplitude of this mode might depend on the choice of gauge in the gravitational case. This requires further analysis, which we do not attempt here. We remind that, from a practical point of view, the linear mode should not cause any real concern, as it cannot affect any gauge-invariant quantity derived from the SF.

\section{ISCO SHIFT}

Using our SF code we can start to explore the $O(\mu)$ "post-geodesic" dynamics of the orbit, and quantify the physical effects resulting from the finiteness of $\mu$. As a first concrete application of the code, we calculated the $O(\mu)$ shift in the location and frequency of the ISCO due to the conservative piece of the SF. We recently reported the results of this calculation in a Letter [33]. Here (and in Appendix E] we provide full details of this analysis.

The radiative transition across the ISCO, from a slow quasi-circular inspiral to a rapid plunge, has been studied in the past and is now well understood. The transition occurs not at a well-defined radius but rather in a gradual manner, across a "transition regime", whose width (in terms of the corresponding azimuthal frequency) is proportional to a low power of the mass ratio: $\Delta \Omega_{\text {trans }} \propto(\mu / M)^{2 / 5}$ [50, 51]. However, apart from the dominant radiative effect which drives the inspiral (and the eventual transition to plunge), the gravitational SF also has a conservative effect, which shifts the location of the ISCO away from $r=6 M$ [by an amount of $O(\mu)$ ]. Unlike the radiative transition, this conservative shift is precisely quantifiable. Moreover, the value of the azimuthal frequency at the shifted ISCO, $\Omega_{\text {isco }}$, is essentially gauge invariant, and hence provides a useful handle on the strong-field conservative dynamics. Indeed, the value of $\Omega_{\text {isco }}$ (for mass ratios not necessarily extreme) has long been utilized in testing and calibrating various approximate treatments of the general-relativistic binary problem (see., e.g., [52, 53], and the very recent [21]). Our $\mathrm{SF}$ code allows us, for the first time, to obtain a precise value for $\Omega_{\text {isco }}$ (modulo a controlled numerical error) at $O(\mu)$.

In what follows we first derive a formula for the ISCO frequency $\Omega_{\text {isco }}$ including $O(\mu)$ conservative SF corrections, and then describe the numerical method used to obtain the necessary SF data, and the results. Many of the details are relegated to Appendix $\mathrm{F}$

\section{A. Formulation}

We first review the notion of ISCO in the unperturbed [geodesic, $O\left(\mu^{0}\right)$ ] case. The radial geodesic equation [obtained by differentiating Eq. (2.2) with respect to $\tau$ ] reads

$$
\frac{d^{2} r_{\mathrm{p}}}{d \tau^{2}}=\mathcal{F}_{\mathrm{eff}}\left(r_{\mathrm{p}}, \mathcal{L}^{2}\right) ; \quad \mathcal{F}_{\mathrm{eff}}\left(r, \mathcal{L}^{2}\right) \equiv-\frac{1}{2} \frac{\partial R\left(r, \mathcal{L}^{2}\right)}{\partial r}
$$

We consider a slightly eccentric orbit representing an $e$-perturbation of a circular orbit with radius $r_{0}$. We write

$$
r_{\mathrm{p}}(\tau)=r_{0}+e r_{1}(\tau)+O\left(e^{2}\right)
$$

where $e \ll 1$ and $r_{1}(\tau)$ is $e$-independent. Substituting this in Eq. (5.1) and reading the $O(e)$ term, we obtain

$$
e \frac{d^{2} r_{1}}{d \tau^{2}}=\left.\frac{\partial \mathcal{F}_{\mathrm{eff}}\left(r_{\mathrm{p}}, \mathcal{L}^{2}\right)}{\partial r_{\mathrm{p}}}\right|_{e=0} e r_{1}+\left.\frac{\partial \mathcal{F}_{\mathrm{eff}}\left(r_{\mathrm{p}}, \mathcal{L}^{2}\right)}{\partial \mathcal{L}^{2}}\right|_{e=0} \delta_{e}\left(\mathcal{L}^{2}\right),
$$

where $\delta_{e}$ denotes a linear variation with respect to $e$ (holding $r_{0}$ fixed). Since $\delta_{e}\left(\mathcal{L}^{2}\right)=0$ for geodesics [see Eq. (2.4)], we obtain

$$
\frac{d^{2} r_{1}}{d \tau^{2}}=-\omega_{r}^{2} r_{1}
$$

with

$$
\omega_{r}^{2}=-\left.\frac{\partial \mathcal{F}_{\mathrm{eff}}\left(r_{\mathrm{p}}, \mathcal{L}^{2}\right)}{\partial r_{\mathrm{p}}}\right|_{e=0}=\frac{M\left(r_{0}-6 M\right)}{r_{0}^{3}\left(r_{0}-3 M\right)} .
$$

Equation (5.4) tells us that the orbit is stable under small-e perturbations whenever $\omega_{r}^{2}>0$, and is unstable under such perturbations when $\omega_{r}^{2}<0$. The innermost stable circular orbit is identified by the condition $\omega_{r}^{2}=0$, giving 
$r_{\text {isco }}=6 M$. We also find that, at $O(e)$, the radial motion is harmonic in $\tau$ : Integrating Eq. (5.4) with the assumption of a periapsis passage at $\tau=0$, we obtain $r_{1}=-r_{0} \cos \omega_{r} \tau$ and hence

$$
r_{\mathrm{p}}(\tau)=r_{0}\left(1-e \cos \omega_{r} \tau\right)+O\left(e^{2}\right)
$$

Next, we consider the $O(\mu)$ correction to the orbit caused by the conservative piece of the SF. The equations of motion become

$$
\begin{gathered}
\frac{d \tilde{\mathcal{E}}}{d \tilde{\tau}}=-\mu^{-1} F_{t}^{\mathrm{cons}}, \quad \frac{d \tilde{\mathcal{L}}}{d \tilde{\tau}}=\mu^{-1} F_{\varphi}^{\mathrm{cons}}, \\
\frac{d^{2} \tilde{r}_{\mathrm{p}}}{d \tilde{\tau}^{2}}=\mathcal{F}_{\mathrm{eff}}\left(\tilde{r}_{\mathrm{p}}, \tilde{\mathcal{L}}^{2}\right)+\mu^{-1} F_{\mathrm{cons}}^{r}
\end{gathered}
$$

where hereafter overtildes indicate quantities associated with the SF-corrected orbit (which is no longer a geodesic). Here we have defined the SF-corrected energy and angular momentum parameters $\tilde{\mathcal{E}}(\tilde{\tau})$ and $\tilde{\mathcal{L}}(\tilde{\tau})$ (in general no longer constants of the motion) through

$$
\frac{d \tilde{t}_{\mathrm{p}}}{d \tilde{\tau}}=\frac{\tilde{\mathcal{E}}}{f\left(\tilde{r}_{\mathrm{p}}\right)}, \quad \frac{d \tilde{\varphi}_{\mathrm{p}}}{d \tilde{\tau}}=\frac{\tilde{\mathcal{L}}}{\tilde{r}_{\mathrm{p}}^{2}}
$$

in analogy with Eqs. (2.1). We assume that the orbit remains bound under the effect of the conservative SF, with $\tilde{r}_{\min } \leq \tilde{r}_{\mathrm{p}}(\tilde{\tau}) \leq \tilde{r}_{\max }$, and once again define $p$ and $e$ as in Eq. (2.3), replacing $r_{\min } \rightarrow \tilde{r}_{\min }$ and $r_{\max } \rightarrow \tilde{r}_{\max }($ we leave $e$ and $p$ untilded for notational brevity). Without loss of generality we take $\tilde{r}_{\mathrm{p}}(\tilde{\tau}=0)=\tilde{r}_{\min }$.

The radial component $F_{\text {cons }}^{r}$ is an even, periodic function of $\tau$ along the geodesic $x_{\mathrm{p}}(\tau)$ [recall Eq. (2.33)], and hence also, at leading order in $\mu$, an even, periodic function of $\tilde{\tau}$ along the perturbed orbit $\tilde{x}_{\mathrm{p}}(\tau)$. [This is because $\tilde{x}_{\mathrm{p}}(\tau)-x_{\mathrm{p}}(\tau) \propto O(\mu)$ while $F_{\text {cons }}^{r}$ is already $O\left(\mu^{2}\right)$.] Since $\tilde{r}_{\mathrm{p}}(\tau)$ too is even and periodic in $\tilde{\tau}$ (and monotonically increasing between $\tilde{r}_{\min }$ and $\left.\tilde{r}_{\max }\right)$, we may express $F_{\text {cons }}^{r}$ as a function of $\tilde{r}_{\mathrm{p}}$ only, for given $p, e: F_{\text {cons }}^{r}=F_{\text {cons }}^{r}\left(\tilde{r}_{\mathrm{p}} ; p, e\right)$. In Eq. (5.8) the quantities $\tilde{r}_{\mathrm{p}}(\tau), d^{2} \tilde{r}_{\mathrm{p}} / d \tilde{\tau}^{2}$ and $F_{\text {cons }}^{r}$ are all periodic and even in $\tau$, and we conclude that $\tilde{\mathcal{L}}$, too, is periodic and even. Hence, we may write $\tilde{\mathcal{L}}=\tilde{\mathcal{L}}\left(\tilde{r}_{\mathrm{p}} ; p, e\right)$.

Let us now specialize to a slightly eccentric (SF-perturbed) orbit. Working through $O(e)$, we write $r_{\text {min }}=r_{0}(1-e)$ and $r_{\max }=r_{0}(1+e)$, where $r_{0}\left[=p M+O\left(e^{2}\right)\right]$ is the radius of the circular orbit about which we perturb. For this orbit we write $\tilde{r}_{\mathrm{p}}(\tau)=r_{0}+e \tilde{r}_{1}(\tau)+O\left(e^{2}\right)[$ as in Eq. $(\underline{5.2})]$, and we have $F_{\text {cons }}^{r}=F_{\text {cons }}^{r}\left(\tilde{r}_{\mathrm{p}} ; r_{0}, e\right)$ and $\tilde{\mathcal{L}}=\tilde{\mathcal{L}}\left(\tilde{r}_{\mathrm{p}} ; r_{0}, e\right)$. At $O\left(e^{0}\right)$ (i.e., at the circular-orbit limit) $\tilde{\mathcal{L}}$ is constant along the orbit from symmetry, and solving Eq. (5.8) with $d^{2} \tilde{r}_{\mathrm{p}} / d \tilde{\tau}^{2}=0$ immediately gives

$$
\tilde{\mathcal{L}}_{0}^{2}=\frac{M r_{0}^{2}}{r_{0}-3 M}\left[1-\frac{r_{0}^{2}}{\mu M} F_{0}^{r}\right]
$$

where hereafter subscripts " 0 " denote circular-orbit values. In particular, we denote by $F_{0}^{r}$ the circular-orbit value of $F_{\text {cons }}^{r}$ (omitting the label 'cons' for brevity). Then, at $O(e)$, Eq. (5.8) yields

$$
e \frac{d^{2} \tilde{r}_{1}}{d \tilde{\tau}^{2}}=\left.\frac{\partial \mathcal{F}_{\mathrm{eff}}\left(\tilde{r}_{\mathrm{p}}, \tilde{\mathcal{L}}_{0}^{2}\right)}{\partial \tilde{r}_{\mathrm{p}}}\right|_{\tilde{r}_{\mathrm{p}}=r_{0}} e \tilde{r}_{1}+\left.\frac{\partial \mathcal{F}_{\mathrm{eff}}\left(r_{0}, \tilde{\mathcal{L}}^{2}\right)}{\partial\left(\tilde{\mathcal{L}}^{2}\right)}\right|_{\tilde{\mathcal{L}}=\tilde{\mathcal{L}}_{0}} \delta_{e} \tilde{\mathcal{L}}^{2}+\mu^{-1} \delta_{e} F_{\text {cons }}^{r},
$$

where we have used $\delta_{e} \tilde{r}_{\mathrm{p}}=e \tilde{r}_{1}$. To evaluate $\delta_{e} \tilde{\mathcal{L}}^{2}$ and $\delta_{e} F_{\text {cons }}^{r}$, we note that the two quantities depend on $e$ both implicitly, thought $r_{\mathrm{p}}\left(\tau ; r_{0}, e\right)$, and explicitly. However, as we showed in Ref. [33], the explicit linear variation of $\tilde{\mathcal{L}}^{2}$ and $F_{\text {cons }}^{r}$ with respect to $e$ (with fixed $r_{0}$ and $r_{\mathrm{p}}$ ) vanishes at $e=0$. Hence we may write $\delta_{e} \tilde{\mathcal{L}}^{2}=e \tilde{r}_{1} d \tilde{\mathcal{L}}^{2} / d \tilde{r}_{\mathrm{p}}$ and $\delta_{e} F_{\mathrm{cons}}^{r}=e \tilde{r}_{1} d F_{\mathrm{cons}}^{r} / d \tilde{r}_{\mathrm{p}}$ (where the derivatives are evaluated at $e=0$ ), resulting in that Eq. (5.11) takes the form

$$
\frac{d^{2} \tilde{r}_{1}}{d \tilde{\tau}^{2}}=-\tilde{\omega}_{r}^{2} \tilde{r}_{1}
$$

with

$$
\tilde{\omega}_{r}^{2}=-\frac{d}{d \tilde{r}_{\mathrm{p}}}\left[\mathcal{F}_{\mathrm{eff}}\left(\tilde{r}_{\mathrm{p}}, \tilde{\mathcal{L}}\left(\tilde{r}_{\mathrm{p}}\right)^{2}\right)+\mu^{-1} F_{\text {cons }}^{r}\left(\tilde{r}_{\mathrm{p}}\right)\right]_{\tilde{r}_{\mathrm{p}}=r_{0}}
$$


To obtain a more explicit expression for the shifted radial frequency $\tilde{\omega}_{r}$, we next expand $\tilde{\mathcal{L}}$ and $F_{\text {cons }}^{r}$ in $e$ through $O(e)$. First, Solving Eq. (5.12) with the initial condition $\tilde{r}_{\mathrm{p}}=\tilde{r}_{\mathrm{min}}$, we find $\tilde{r}_{1}=-r_{0} \cos \tilde{\omega}_{r} \tilde{\tau}$. Then we expand $F_{\mathrm{cons}}^{r}=F_{0}^{r}+e \tilde{r}_{1}\left(d F_{\mathrm{cons}}^{r} / d \tilde{r}_{\mathrm{p}}\right)_{\tilde{r}_{\mathrm{p}}=r_{0}}+O\left(e^{2}\right)$, giving

$$
F_{\mathrm{cons}}^{r}=F_{0}^{r}+e F_{1}^{r} \cos \tilde{\omega}_{r} \tilde{\tau}+O\left(e^{2}\right)
$$

where we have denoted $F_{1}^{r} \equiv-r_{0}\left(d F_{\text {cons }}^{r} / d \tilde{r}_{\mathrm{p}}\right)_{\tilde{r}_{\mathrm{p}}=r_{0}}$. In a similar manner we expand $\tilde{\mathcal{L}}=\tilde{\mathcal{L}}_{0}+e \tilde{r}_{1}\left(d \tilde{\mathcal{L}} / d \tilde{r}_{\mathrm{p}}\right) \tilde{r}_{\mathrm{p}}=r_{0}+$ $O\left(e^{2}\right)$, which, in conjunction with Eq. (5.7), gives

$$
F_{\varphi}^{\mathrm{cons}}=e \tilde{\omega}_{r} F_{\varphi}^{1} \sin \tilde{\omega}_{r} \tilde{\mathcal{\tau}}+O\left(e^{2}\right)
$$

where $F_{\varphi}^{1} \equiv \mu r_{0}\left(d \tilde{\mathcal{L}} / d \tilde{r}_{\mathrm{p}}\right)_{\tilde{r}_{\mathrm{p}}=r_{0}}$. Finally, using Eqs. (5.14) and (15.15) and substituting for $\tilde{\mathcal{L}}_{0}$ from Eq. (5.10), Eq. (5.13) becomes

$$
\begin{aligned}
\tilde{\omega}_{r}^{2} & =\omega_{r}^{2}-\frac{3\left(r_{0}-4 M\right)}{r_{0}\left(r_{0}-3 M\right)} \mu^{-1} F_{0}^{r}+\frac{1}{r_{0}} \mu^{-1} F_{1}^{r}-\frac{2}{r_{0}^{4}} \sqrt{M\left(r_{0}-3 M\right)} \mu^{-1} F_{\varphi}^{1} \\
& =\frac{M}{r_{0}^{3}\left(r_{0}-3 M\right)}\left[r_{0}-6 M-\frac{3 r_{0}^{2}\left(r_{0}-4 M\right)}{M \mu} F_{0}^{r}+\frac{r_{0}^{2}\left(r_{0}-3 M\right)}{M \mu} F_{1}^{r}-\frac{2\left(r_{0}-3 M\right) \sqrt{M\left(r_{0}-3 M\right)}}{M r_{0} \mu} F_{\varphi}^{1}\right] .
\end{aligned}
$$

This formula describes the $O(\mu)$ conservative shift in the radial frequency off its geodesic value. Note that it requires knowledge of the SF through $O(e)$ (knowledge of the circular-orbit SF is not sufficient).

The perturbed ISCO radius, $r=\tilde{r}_{\text {isco }}$, is now obtained from the condition $\tilde{\omega}_{r}^{2}\left(r_{0}=\tilde{r}_{\text {isco }}\right)=0$. Namely, $\tilde{r}_{\text {isco }}$ is the value of $r_{0}$ that nullifies the expression in square brackets in the second line of Eq. (5.16) through $O(\mu)$. Note that in this expression we are allowed to substitute $r_{0}=r_{\text {isco }}=6 M$ in all SF terms, since such terms are already $O(\mu)$ [so the error introduced affects $\tilde{r}_{\text {isco }}$ only at $\left.O\left(\mu^{2}\right)\right]$. Thus, we readily obtain

$$
\begin{aligned}
\Delta r_{\mathrm{isco}} & \equiv \tilde{r}_{\mathrm{isco}}-6 M \\
& =\left(M^{2} / \mu\right)\left(216 F_{0 \mathrm{is}}^{r}-108 F_{1 \mathrm{is}}^{r}+\sqrt{3} M^{-2} F_{\varphi \mathrm{is}}^{1}\right)
\end{aligned}
$$

through $O(\mu)$, where we have denoted $F_{0 \text { is }}^{r} \equiv F_{0}^{r}\left(r_{0}=6 M\right)$ and similarly for $F_{1 \text { is }}^{r}, F_{\varphi \text { is }}^{1}$.

Since the coordinate ISCO shift $\Delta r_{\text {isco }}$ is gauge dependent (just like the SF itself), it is not very useful as a benchmark for comparisons. Instead, we now consider the (SF-corrected) circular-orbit azimuthal frequency,

$$
\tilde{\Omega} \equiv \frac{d \tilde{\varphi}_{\mathrm{p}}}{d \tilde{t}}=\frac{d \tilde{\varphi}_{\mathrm{p}} / d \tilde{\tau}}{d \tilde{t}_{\mathrm{p}} / d \tilde{\tau}}
$$

which, as discussed in [19], is invariant under all $O(\mu)$ gauge transformations whose generators respect the helical symmetry of the circular-orbit configuration. Using Eqs. (5.9) and (5.10) we obtain

$$
\tilde{\Omega}=\Omega\left[1-\frac{r_{0}\left(r_{0}-3 M\right)}{2 M \mu f_{0}} F_{0}^{r}\right],
$$

where $\Omega \equiv\left(M / r_{0}^{3}\right)^{1 / 2}$ is the geodesic (no SF) value. Evaluated at $r_{0}=\tilde{r}_{\text {isco }}$, the SF-induced frequency shift $\Delta \Omega \equiv \tilde{\Omega}-\Omega$ reads [through $O(\mu)$ ]

$$
\Delta \Omega_{\text {isco }}=-\frac{1}{6^{3 / 2} M}\left[\frac{\Delta r_{\text {isco }}}{4 M}+\frac{27 M}{2 \mu} F_{0 \text { is }}^{r}\right] .
$$

Equations analogous to (5.17) and (5.20) were obtained by Diaz-Rivera et al. [10] in their study of scalar SF effects.

Despite the fact that $\Delta \Omega_{\text {isco }}$ is gauge invariant (in the above sense), care must be exercised in interpreting the quantity expressed in Eq. (5.20). As pointed out in [26], the Lorenz-gauge metric perturbation has the somewhat peculiar feature that its $t t$ component (in Schwarzschild coordinates) does not fall to zero as $r \rightarrow \infty$, but instead $h_{t t} \rightarrow-2 \alpha(=\mathrm{const})$, with $\alpha=\mu\left[r_{0}\left(r_{0}-3 M\right)\right]^{-1 / 2}$. This peculiarity can be removed simply by "rescaling" the time coordinate as $t \rightarrow \hat{t}=(1+2 \alpha)^{1 / 2} t \cong(1+\alpha) t$ [neglecting terms of $O\left(\mu^{2}\right)$ ]. The angular frequency $\tilde{\Omega}$, whose definition has an explicit reference to $t$, will be modified under such rescaling as $\tilde{\Omega} \rightarrow \hat{\tilde{\Omega}}=(1-\alpha) \tilde{\Omega}$. As explained in Ref. [19], and recently emphasized by Damour [21], in order to compare with the frequency derived in a gauge in which $h_{t t}$ admits the ordinary asymptotic fall off [such as the Regge-Wheeler gauge, or the gauge associated with the $O(\mu)$ 
part of EOB theory], one must not use the "Lorenz-gauge" frequency $\tilde{\Omega}$, but rather the $t$-rescaled frequency $\hat{\tilde{\Omega}}$. For practical reasons, therefore, we also give here the "t-rescaled" version of Eq. (5.20):

$$
\Delta \hat{\Omega}_{\mathrm{isco}}=-\frac{1}{6^{3 / 2} M}\left[\frac{\Delta r_{\mathrm{isco}}}{4 M}+\frac{27 M}{2 \mu} F_{0 \mathrm{is}}^{r}+\frac{\mu}{\sqrt{18} M}\right],
$$

where we have used $\alpha\left(r_{0}=6 M\right)=(\mu / M) / \sqrt{18}$.

\section{B. Numerical method and results}

In view of Eqs. (5.17) and (5.20), the task of calculating $\Delta r_{\text {isco }}$ and $\Delta \Omega_{\text {isco }}$ amounts to obtaining numerical values for the three coefficients $F_{0 \text { is }}^{r}, F_{1 \text { is }}^{r}$ and $F_{\varphi \text { is }}^{1}$. The first coefficient is easily obtained: $F_{0 \text { is }}^{r}$ is just the SF along a strictly circular geodesic with radius $r_{0}=6 M$, and we already computed it in Paper I using our circular-orbit code (it is one of the values listed in Table $\mathrm{V}$ of [15]). Here we repeat this calculation at greater numerical precision, obtaining

$$
F_{0 \text { is }}^{r}=0.0244665(1) \mu / M^{2} \text {. }
$$

This is consistent with Berndtson's [18] result of $0.024466497 \mu / M^{2}$.

The computation of $F_{\varphi \text { is }}^{1}$ and $F_{1 \text { is }}^{r}$ is much more delicate, as it requires to resolve numerically the small variation in the SF under a small-e perturbation of a circular orbit [recall Eqs. (5.14) and (5.15)]. We are not helped by the fact that this variation need be evaluated at $(p, e)=(6,0)$, which is a singular point in the $p-e$ plane (see below). The subtlety of the computation task calls for an extra caution, so, as a safeguard measure, we devised and implemented two completely independent strategies for evaluating $F_{\varphi \text { is }}^{1}$ and $F_{1 \text { is }}^{r}$. The first, more direct approach $(\operatorname{method} I)$ involves an evaluation of the SF along a sequence of eccentric geodesics approaching the ISCO along a suitable curve in the $p$-e plane; the required SF coefficients are then extracted as certain orbital integrals, extrapolated to $e \rightarrow 0$ (see below). The second strategy (method $I I$ ) involves an expansion of the field equations themselves about a circular orbit, through $O(e)$. In what follows we describe each of the two methods and their outcomes in turn.

\section{Method I: extrapolation in the p-e plane}

From Eqs. (5.14) and (5.15) we obtain

$$
F_{1 \text { is }}^{r}=\lim _{p \rightarrow 6} \lim _{e \rightarrow 0} \hat{F}_{1}^{r}(p, e), \quad \hat{F}_{1}^{r}(p, e) \equiv 2 \omega_{r}(e \pi)^{-1} \int_{0}^{\pi / \omega_{r}} F_{\text {cons }}^{r} \cos \omega_{r} \tau d \tau
$$

and

$$
F_{\varphi \mathrm{is}}^{1}=\lim _{p \rightarrow 6} \lim _{e \rightarrow 0} \hat{F}_{\varphi}^{1}(p, e), \quad \hat{F}_{\varphi}^{1}(p, e) \equiv 2(e \pi)^{-1} \int_{0}^{\pi / \omega_{r}} F_{\varphi}^{\mathrm{cons}} \sin \omega_{r} \tau d \tau
$$

where $\omega_{r}, \tau, F_{\text {cons }}^{r}$ and $F_{\varphi}^{\text {cons }}$ are the values corresponding to a geodesic with parameters $p, e$ (hence we were allowed to remove the tilde symbols off $\omega_{r}$ and $\left.\tau\right)$. It may be noticed that, formally, the quantities $\hat{F}_{1}^{r}(p, e)$ and $\hat{F}_{\varphi}^{1}(p, e)$ are inverse Fourier integrals describing the first $\omega_{r}$-harmonic of $F_{\text {cons }}^{r}$ and $F_{\varphi}^{\text {cons }}$, respectively. The latter two quantities, recall, are both periodic in $\tau$ along the geodesic, with $F_{\text {cons }}^{r}$ being even and $F_{\varphi}^{\text {cons }}$ being odd in $\tau$.

The order of the limits in Eqs. (5.23) and (5.24) is very important: Since $F_{1 \text { is }}^{r}$ and $F_{\varphi \text { is }}^{1}$ are defined through an expansion about a stable circular orbit $(e=0)$, we must first take the limit $e \rightarrow 0$ before taking $p \rightarrow 6$. In practice, however, it is more computationally economical to approach the point $(p, e)=(6,0)$ along a certain continuous curve in the $p-e$ plane, rather than having to extrapolate to $e \rightarrow 0$ along several different $p=$ const lines and then extrapolate the resulting data again to $p \rightarrow 6$. In doing so, however, we must choose our curve carefully, since the limiting point $(6,0)$ is known to be a singular one. [A simple manifestation of this singularity is the fact that the rate at which the radial frequency $\omega_{r}$ vanishes at the limit $(p, e) \rightarrow(6,0)$ depends upon the direction in the $p-e$ plane from which this limit is taken-see, e.g., Eq. (2.36) of [38].] In particular, the curve must always "stay away" from the separatrix $p=6+2 e$, where the $e$-expansions (5.14) and (5.15) [on which Eqs. (5.23) and (5.24) rely] are meaningless. As discussed by Cutler et al. in [38] (in a slightly different context), in order for the e-expansion to hold, one must require not only $e \ll 1$ but also $e \ll p-6$. For our purpose, the point $(6,0)$ must be approached keeping $e \ll \min \{1, p-6\}$.

Here we pick the curve $p=6+\sqrt{e}$, for which $e \ll 1$ automatically implies $e \ll p-6$. We select a set of $p$ values approaching $p \rightarrow 6$, and for each value we use our code to calculate the SF along an eccentric geodesic with 
parameters $\left(p, e_{c}(p)\right)$, where $e_{c}(p)=(p-6)^{2}$. For each of these parameter-space points we then construct the quantities $\hat{F}_{1}^{r}\left(p, e_{c}(p)\right)$ and $\hat{F}_{\varphi}^{1}\left(p, e_{c}(p)\right)$ defined in Eqs. (5.23) and (5.24), by numerically integrating the SF data along the orbit. The results are shown in Fig. 9, Finally, we obtain the desired coefficients $F_{1 \text { is }}^{r}$ and $F_{\varphi \text { is }}^{1}$ by extrapolating the numerical values of $\hat{F}_{1}^{r}$ and $\hat{F}_{\varphi}^{1}$ to $p=6$. We do this, in practice, by fitting a cubic polynomial to the numerical data, writing (for example) $\hat{F}_{1}^{r}=a_{0}+a_{1}(p-6)+\cdots+a_{3}(p-6)^{3}$ and taking the value of the fitting coefficient $a_{0}$ as our approximation for $F_{1 \text { is }}^{r}$. The results are

$$
\begin{aligned}
& F_{1 \text { is }}^{r}=0.0620(5) \mu / M^{2}, \\
& F_{\varphi \text { is }}^{1}=-1.066(1) \mu \quad(\operatorname{method} I) .
\end{aligned}
$$

Here, as elsewhere in this work, a parenthetical figure indicates the uncertainty in the last displayed decimal place due to numerical error [so, e.g., 0.0620(5) stands for $0.0620 \pm 0.0005$ ].

The error in the above calculation is estimated as follows. For each value of $e$ considered, we first estimate the numerical error in each of $\hat{F}_{1}^{r}$ and $\hat{F}_{\varphi}^{1}$ as the difference between the value obtained with the finest numerical grid used and the value obtained with a coarser grid (of four times the cell area). We calculate this difference for each of the $l$-mode contributions to $\hat{F}_{1}^{r}$ and $\hat{F}_{\varphi}^{1}$, and conservatively take the total ( $l$-summed) error as the sum over the moduli of the individual $l$-mode errors (adding to this the large- $l$ tail extrapolation error). The "error bars" thus obtained are those displayed in Fig. 9. In the final step we fit a cubic polynomial curve to each of the $\hat{F}_{1}^{r}, \hat{F}_{\varphi}^{1}$ data sets, using the above error bars as a fitting weight. We take the standard fitting error in the constant term (the above coefficient $\left.a_{0}\right)$ as our estimate for the error in $F_{1 \text { is }}^{r}, F_{\varphi \text { is }}^{1}$. It is this estimated error that we indicate in Eqs. (5.25).
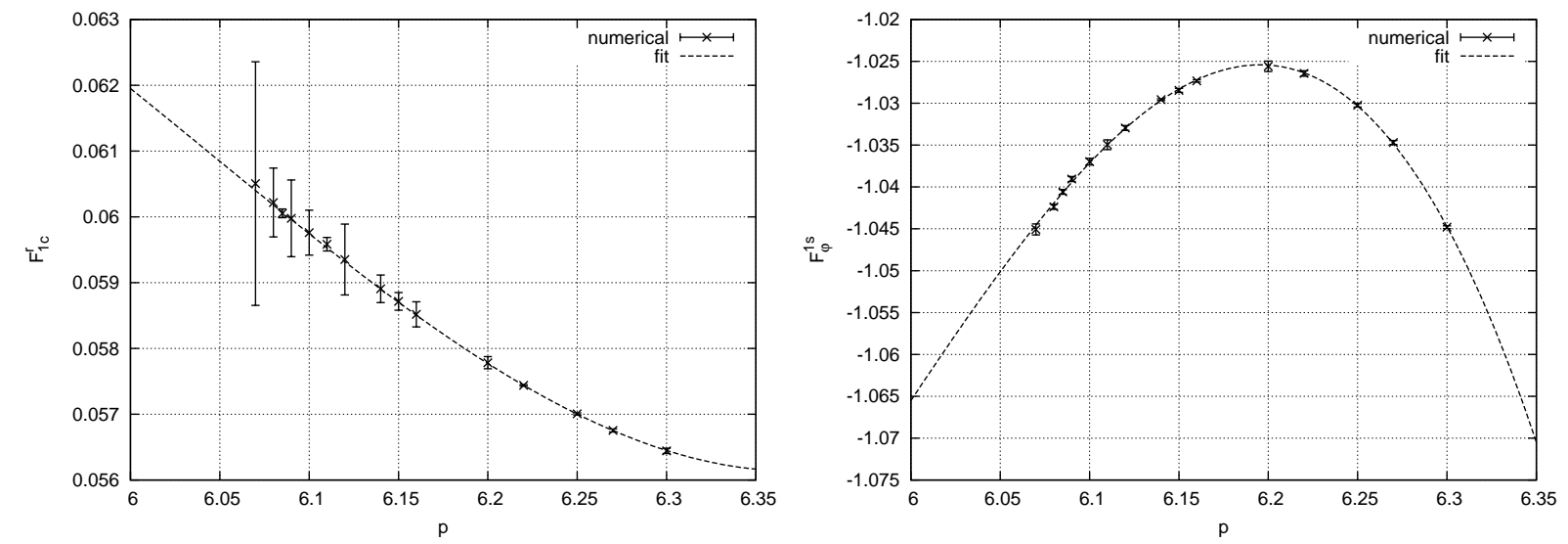

FIG. 9: Derivation of the SF coefficients $F_{1 \text { is }}^{r}$ and $F_{\varphi \text { is }}^{1}$ entering the ISCO-shift formula (5.17), using method $I$. Plotted are the numerical values of $\hat{F}_{1}^{r}(p, e)$ and $\hat{F}_{\varphi}^{1}(p, e)$ [see Eqs. (15.23) and (5.24)] for a sequence of points in the $p-e$ plane approaching the ISCO along the curve $p=6+\sqrt{e}$. Error bars indicate the estimated numerical error, which is evaluated as explained in the text. The solid curves are weighted polynomial fits used to extrapolate the values of $\hat{F}_{1}^{r}$ and $\hat{F}_{\varphi}^{1}$ to $p \rightarrow 6$. The extrapolated values are the desired coefficients $F_{1 \text { is }}^{r}$ and $F_{\varphi \text { is }}^{1}$.

\section{Method II: e-expansion of the field equations}

Our second procedure for evaluating $F_{1 \text { is }}^{r}$ and $F_{\varphi \text { is }}^{1}$ is based on a systematic expansion of the field equations (and the SF) through $O(e)$, and is similar, in principle, to the method used in Ref. [10] for the scalar-field SF. The idea is to consider a slightly eccentric geodesic, with $r_{\mathrm{p}}(\tau)=r_{0}\left(1-e \cos \omega_{r} \tau\right)$ where $e \ll 1$ and $r_{0}=$ const [as in Eq. (5.6)], and for this geodesic expand the source term in the perturbation equations (2.20) through $O(e)$ as

$$
S^{(i)}(t, r) \delta\left(r-r_{\mathrm{p}}\right)=S_{0}^{(i)}\left(t, r_{0}\right) \delta\left(r-r_{0}\right)+e\left[S_{1}^{(i)}\left(t, r_{0}\right) \delta\left(r-r_{0}\right)+T_{1}^{(i)}\left(t, r_{0}\right) \delta^{\prime}\left(r-r_{0}\right)\right]
$$

Here we have omitted the indices $l m$ for brevity and used a prime to denote $d / d r$. The various expansion coefficients are given by

$$
S_{0}^{(i)} \equiv S^{(i)}\left(t, r_{0}\right),\left.\quad S_{1}^{(i)} \equiv \frac{\partial S^{(i)}\left(t, r_{\mathrm{p}}\right)}{\partial e}\right|_{e=0}, \quad T_{1}^{(i)} \equiv-\left.S^{(i)}\left(t, r_{0}\right) \frac{\partial r_{\mathrm{p}}}{\partial e}\right|_{e=0}=S_{0}^{(i)} r_{0} \cos \omega_{r} \tau
$$



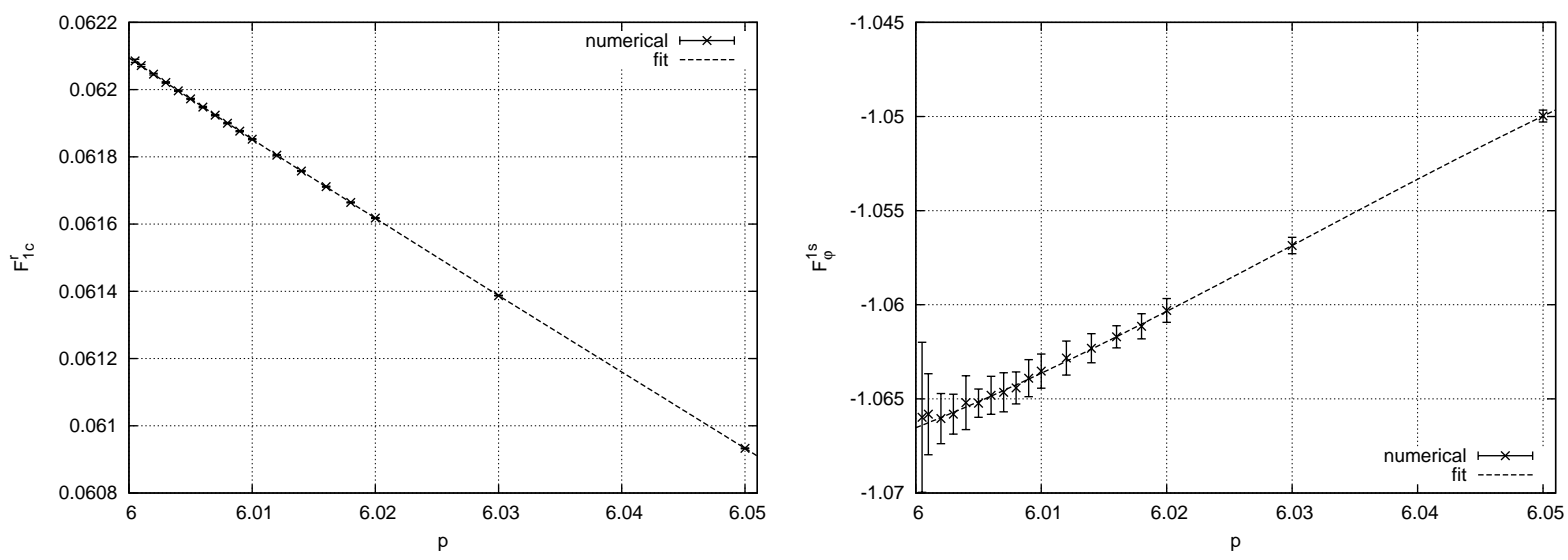

FIG. 10: Derivation of $F_{1 \text { is }}^{r}$ and $F_{\varphi \text { is }}^{1}$, using method II. Plotted are the numerical values of $F_{\text {cos }}^{r}(p)$ and $F_{\varphi}^{\sin }(p)$ [see Eqs. (5.33) and [5.35] ] for a sequence of points approaching the ISCO along the $p$-axis in the $p-e$ plane. Error bars indicate the estimated numerical error, which is evaluated as explained in the text. The solid curves are weighted polynomial fits used to extrapolate the values of $F_{\cos }^{r}$ and $F_{\varphi}^{\sin }$ to $p \rightarrow 6$. The extrapolated values are the desired coefficients $F_{1 \text { is }}^{r}$ and $F_{\varphi \text { is }}^{1}$.

The coefficients $S_{1}^{(i)}$ (for $\left.i=1, \ldots, 10\right)$ are evaluated by substituting the above expression for $r_{\mathrm{p}}(\tau)$ in Eqs. (B11)(B20), along with $u^{r}=e r_{0} \omega_{r} \sin \omega_{r} \tau+O\left(e^{2}\right), \mathcal{E}=\left(r_{0}-2 M\right)\left[r_{0}\left(r_{0}-3 M\right)\right]^{-1 / 2}+O\left(e^{2}\right)$ and $\mathcal{L}=r_{0}\left(r_{0} / M-3\right)^{-1 / 2}+O\left(e^{2}\right)$, and then expanding through $O(e)$. Explicit expressions for the $S_{1}^{(i)}$ 's are given in Appendix $\mathbb{F}$ We also formally expand the metric perturbation functions $\bar{h}^{(i) l m}$ in the form

$$
\bar{h}^{(i)}(t, r)=\bar{h}_{0}^{(i)}(t, r)+e \bar{h}_{1}^{(i)}(t, r)+O\left(e^{2}\right)
$$

(again omitting the indices $l m$ for brevity), and consequently single out the $O\left(e^{0}\right)$ and $O\left(e^{1}\right)$ pieces of the perturbation equations (2.20) as

$$
\begin{aligned}
& \square \bar{h}_{0}^{(i)}+\mathcal{M}_{(j)}^{(i)} \bar{h}_{0}^{(j)}=S_{0}^{(i)} \delta\left(r-r_{0}\right), \\
& \square \bar{h}_{1}^{(i)}+\mathcal{M}_{(j)}^{(i)} \bar{h}_{1}^{(j)}=S_{1}^{(i)} \delta\left(r-r_{0}\right)+T_{1}^{(i)} \delta^{\prime}\left(r-r_{0}\right) .
\end{aligned}
$$

Notice that the source terms in these equations are evaluated along a circular geodesic (of radius $r_{0}$ ). The function $\bar{h}_{0}^{(i)}$ is just the physical Lorenz-gauge perturbation from this circular geodesic. The function $\bar{h}_{1}^{(i)}$ is not a physical perturbation; in particular, it is discontinuous across the orbit (due to the $\delta^{\prime}$ term in its source).

For our purpose we need to solve Eqs. (5.29) and (5.30) for $r_{0}=6 M$. While there is no problem solving for $\bar{h}_{0}^{(i)}$ (as we in fact already did in Paper I), solving for $\bar{h}_{1}^{(i)}$ is numerically subtle, since the source functions $S_{1}^{(i)}$ become singular as $\omega_{r} \rightarrow 0$ [cf. Eqs. (F2) - (F12) in Appendix E. Hence, as in method I, we use extrapolation: we first solve Eq. (5.30) for a sequence of $r_{0}$ values approaching $6 M$, and then extrapolate our data to $r_{0}=6 M$.

A second difficulty arises because our TD algorithm assumes that the numerical variables are continuous on the particle's orbit. This is no longer the case for the functions $\bar{h}_{1}^{(i)}$, which suffer finite jump discontinuities across the orbit. This requires an adaptation of the junction conditions implemented in the finite-difference scheme. We describe the suitably modified junction conditions in Appendix $\mathrm{F}$. We use our amended TD algorithm to solve for the functions $\bar{h}_{1}^{(i)}$ via time evolution.

Once the functions $\bar{h}_{0}^{(i)}(t, r)$ and $\bar{h}_{1}^{(i)}(t, r)$ are at hand we can proceed to construct the SF along the slightly eccentric orbit through $O(e)$. Here we need to be cautious: When we evaluate $\bar{h}^{(i)}(t, r)$ (and its derivatives) along the slightly eccentric orbit $r_{\mathrm{p}}(\tau)$, we must take proper account of the $O(e)$ contribution coming from the term $\bar{h}_{0}^{(i)}\left(t, r_{\mathrm{p}}\right)$ via the expansion of $r_{\mathrm{p}}$ in $e$. Recalling Eq. (5.28) we have, in fact,

$$
\bar{h}^{(i)}\left(t, r_{\mathrm{p}}\right)=\bar{h}_{0}^{(i)}\left(t, r_{0}\right)+e\left[\bar{h}_{1}^{(i)}\left(t, r_{0}\right)-\bar{h}_{0, r}^{(i)}\left(t, r_{0}\right) r_{0} \cos \omega_{r} \tau\right]+O\left(e^{2}\right),
$$

with similar expansions applying to the $r$ and $t$ derivatives of the perturbation along $r_{\mathrm{p}}(\tau)$ :

$$
\bar{h}_{, \sigma}^{(i)}\left(t, r_{\mathrm{p}}\right)=\bar{h}_{0, \sigma}^{(i)}\left(t, r_{0}\right)+e\left[\bar{h}_{1, \sigma}^{(i)}\left(t, r_{0}\right)-\bar{h}_{0, r \sigma}^{(i)}\left(t, r_{0}\right) r_{0} \cos \omega_{r} \tau\right]+O\left(e^{2}\right)
$$


where $\sigma=r$ or $t$. Recall that the derivatives of $\bar{h}_{0}^{(i)}$ and $\bar{h}_{1}^{(i)}$, as well as the function $\bar{h}_{1}^{(i)}$ itself, are discontinuous at $r_{0}$ and hence defined only in the sense of a directional limit $r \rightarrow r_{0}^{ \pm}$(however, for simplicity we choose here not to reflect this with a suitable notation). Notice also that the first derivatives of the metric perturbation $\bar{h}^{(i)}$ along the slightly eccentric geodesic involve second derivatives of the function $\bar{h}_{0}^{(i)}$ along the circular geodesic (an $r r$ derivative for $\bar{h}_{, r}^{(i)}$ and an $r t$ derivative for $\left.\bar{h}_{, t}^{(i)}\right)$. The necessary data for our calculation therefore includes the functions $\bar{h}_{0}^{(i)}$ and $\bar{h}_{1}^{(i)}$ themselves, as well as the derivatives $\bar{h}_{0, r}^{(i)}, \bar{h}_{0, t}^{(i)} \bar{h}_{1, r}^{(i)}, \bar{h}_{1, t}^{(i)}, \bar{h}_{0, r r}^{(i)}$ and $\bar{h}_{0, r t}^{(i)}$, all evaluated (in a one-sided fashion) at the circular orbit with radius $r_{0}=6 M$.

To construct the SF through $O(e)$, we substitute Eq. (5.28) in Eq. (2.25), and evaluate the outcome along the slightly eccentric orbit $r_{\mathrm{p}}(\tau)$. We remind that the functions $\mathcal{F}$ appearing in Eq. (2.25) are given explicitly in Appendix C in terms of the perturbation $\bar{h}^{(i)}(t, r)$ and its $r$ and $t$ derivatives. We next expand Eq. (2.25) in $e$ through $O(e)$ using the expansions (5.31) and (5.32) and the above $e$-expansions for $u^{r}, \mathcal{E}$ and $\mathcal{L}$. Keeping only the $O(e)$ terms, we proceed through the mode-sum regularization procedure as described in Sec. IIE and construct the $O(e)$ pieces of the conservative SF components $F_{\text {cons }}^{r}$ and $F_{t}^{\text {diss }}$. We find that these $O(e)$ pieces contain only terms proportional to either $\cos \omega_{r} \tau$ or $\sin \omega_{r} \tau$. More precisely, we obtain

$$
\begin{aligned}
& O(e) \text { piece of } F_{\text {cons }}^{r}=e F_{\text {cos }}^{r} \cos \omega_{r} \tau \\
& O(e) \text { piece of } F_{t}^{\text {cons }}=e \omega_{r} F_{t}^{\sin } \sin \omega_{r} \tau
\end{aligned}
$$

where the coefficients $F_{\text {cos }}^{r}$ and $F_{t}^{\sin }$ are constructed from $\bar{h}_{1}^{(i)}, \bar{h}_{1, \sigma}^{(i)}, \bar{h}_{0, \sigma}^{(i)}$ and $\bar{h}_{0, r \sigma}^{(i)}$. (The explicit form of these coefficients is rather complicated and will not be shown here. The coefficients can be readily evaluated using computer algebra tools.) The $O(e)$ part of the orthogonality condition $u^{\alpha} F_{\alpha}=0$ then also gives

$$
O(e) \text { piece of } F_{\varphi}^{\mathrm{cons}}=e \omega_{r} F_{\varphi}^{\sin } \sin \omega_{r} \tau
$$

where $F_{\varphi}^{\sin }$ is related to the numerically-computed coefficients $F_{t}^{\sin }$ and $F_{0}^{r}$ through

$$
F_{\varphi}^{\sin }=-\frac{r_{0}^{2}}{\mathcal{L}_{0} f_{0}}\left(\mathcal{E}_{0} F_{t}^{\mathrm{sin}}+r_{0} F_{0}^{r}\right) .
$$

Comparing Eqs. (5.33) and (5.35) with Eqs. (5.14) and (5.15), one finally identifies $F_{1}^{r}=F_{\cos }^{r}$ and $F_{\varphi}^{1}=F_{\varphi}^{\sin }$.

We have implemented the above procedure for a dozen or so radii $r_{0}$ near $6 M$, and for each radius obtained the values of $F_{1}^{r}$ and $F_{\varphi}^{1}$. We then extrapolated these values to $r_{0}=6 M$ by fitting a cubic polynomial (see Fig. 10). We obtained

$$
\begin{aligned}
& F_{1 \text { is }}^{r}=0.062095(1) \mu / M^{2}, \\
& F_{\varphi \text { is }}^{1}=-1.0665(8) \mu \quad(\operatorname{method} I I) .
\end{aligned}
$$

These values are in agreement, within the estimated numerical accuracy, with the values obtained using method I [compare with Eqs. (5.25)]. Using method II we were able to explore orbits much nearer to the ISCO, resulting in a much improved accuracy. Of course, the agreement between our two independent calculations provides significant reassurance.

With the SF coefficient values given in Eqs. (5.22) and (5.37), formulas (5.17), (5.20) and (5.21) finally yield

$$
\begin{aligned}
\Delta r_{\text {isco }} & =-3.269(2) \mu, \\
\Delta \Omega_{\text {isco }} & =\Omega_{\text {isco }} \times 0.4869(4) \mu / M, \\
\Delta \hat{\Omega}_{\text {isco }} & =\Omega_{\text {isco }} \times 0.2512(4) \mu / M,
\end{aligned}
$$

where $\Omega_{\text {isco }}=\left(6^{3 / 2} M\right)^{-1}$ is the unperturbed (geodesic) value of $\Omega$ at $r=6 M$. The values obtained here are slightly more accurate than the ones we give in our Letter [33] $\left[\Delta r_{\text {isco }}=-3.269(3) \mu\right.$ and $\left.\Delta \Omega_{\text {isco }}=\Omega_{\text {isco }} \times 0.4870(6) \mu / M\right]$, an improvement made possible by the inclusion of additional numerical data points in our analysis.

\section{CONCLUDING REMARKS AND FUTURE APPLICATIONS}

This work marks a new frontline in the program to model realistic two-body inspirals in the extreme mass-ratio regime. For the first time we are able to calculate the full $\left[O\left(\mu^{2}\right)\right]$ gravitational SF across (essentially) the entire parameter space of strong-field bound geodesics in Schwarzschild spacetime. This work also represents a first complete 
end-to-end implementation of a range of computational techniques which were developed gradually over the past decade: mode-sum regularization scheme [29], the 1+1D Lorenz-gauge perturbation formalism [26], and the method of extended homogeneous solutions [31]. As the reader may appreciate, the underlying computational challenge is rather daunting, given the complexity of the field equations, the technical subtleties involved in dealing with the delta-function source, the high computational cost, and the need to patch together different techniques in both the time and frequency domains. Our eventual working code is of considerable complexity and took over two years to develop and test.

Following is a summary of the various tests which helped us establish confidence in our code's performance. (i) The mode-sum regularization procedure is self-validating, in the sense that it is extremely sensitive to errors in the computation of the perturbation multipoles (especially the high- $l$ ones, which are most computationally demanding). If the regularized mode sum shows the expected fall-off behavior at large $l$, this by itself is a strong indication that the high-l modes were calculated correctly. (ii) The code reproduces the known results in the circular-orbit case; these results are now confirmed by 3 independent analyses [15, 17, 18]. (iii) Our evolution code reproduces the correct asymptotic fluxes of gravitational-wave energy and angular momentum, as verified by comparing with results in the literature. (iv) The work done by the dissipative piece of the computed SF is found to precisely balance these fluxes. (v) The value of the ISCO frequency shift derived from our SF seems consistent with the value derived in EOB at 3rd post-Newtonian (PN) order: Damour recently showed that the latter is about $72.5 \%$ of the SF value, with the difference likely attributed to higher-order PN terms [21].

In principle, our code can return the SF along any bound geodesic in Schwarzschild geometry, although in practice computational cost may becomes prohibitive when the orbital period is too large (i.e., for very large $p$ and/or $e$ close to unity). The "workable domain" of our code using a current-day standard single-processor desktop computer is roughly $0 \lesssim e \lesssim 0.5$ and $p \lesssim 20 M$ if a fractional accuracy of $<10^{-4}$ in the SF is sought. The current algorithm incorporates an explicit reference to the radial frequency parameter, so it cannot be used to tackle unbound orbits. However, it may be adapted with moderate effort to handle unbound orbits (including orbits below the last stable orbit) as well.

Even within the above "workable domain", the current code is discouragingly slow. It takes a few days to compute the SF along a single strong-field geodesic, which makes it impractical to cover the entire parameter space of inspirals at sufficient resolution (having in mind the development of theoretical gravitational waveform templates for astrophysical inspirals). There are, however, various ways in which one may improve the computational efficiency and speed. Most obvious, one can use distributed computing - our algorithm should be easily amenable for distribution on a cluster, since different $l$ modes can be calculated in parallel. Other natural approaches include the use of mesh refinement (see Thornburg's recent report [54]) and/or higher-order finite-difference schemes. One may also seek to reduce the computational cost attached to the initial stage of the numerical evolution (when spurious initial waves dominate) by iteratively improving the initial conditions for the evolution - this idea is already being implemented successfully in a 2+1D framework [55]. Other ideas represent a more significant deviation from our approach: (i) work entirely in the frequency domain, making full use of the method of extended homogeneous solutions [31] (this is likely to prove most efficient with smaller eccentricities); or (ii) abandon finite differencing altogether and instead use finite elements or other pseudospectral techniques [56, 57], benefiting from their natural flexibility in accommodating multiple lengthscales.

Nonetheless, some interesting applications are already possible with the current version of our code, and we have presented one of them in Sec. $\mathrm{V}$. Our computation of the ISCO frequency shift represents the first physically-meaningful new result coming out of the SF program, and it has already informed both Numerical-Relativistic calculations [34, 35] and EOB/PN theory [21]. Further ideas for SF/EOB synergy were recently discussed by Damour in [21]. In particular, Damour showed that a computation of the (gauge invariant) conservative SF correction to the precession rate of the periapsis, for slightly eccentric orbits, will give access to the presently unknown 4PN (and possibly higher-order) parameters of EOB theory. We are currently working to extract the necessary SF data to facilitate this calculation [these are essentially the coefficients $F_{0}^{r}, F_{1}^{r}$ and $F_{\varphi}^{1}$ of Eqs. (5.14) and (5.15), as functions of $r_{0}$ ]. Our preliminary results show excellent agreement with the analytic EOB predictions at $2 \mathrm{PN}$ and $3 \mathrm{PN}$, and we are hoping to publish these findings elsewhere [58].

More generally, our code allows to tackle the calculation of post-geodesic $[O(\mu)]$ precession effects at any eccentricity, not necessarily small. This would not only provide a handle on the ' $Q$ ' function of EOB theory, but can also directly inform the computation of conservative effects in inspiral trajectories. Information on the periastron advance as a function of $p, e$ could, for example, be incorporated into an orbital evolution scheme à la Pound \& Poisson [24]. We are currently investigating this direction.

Finally, we comment briefly on the possibility of extending this work to the Kerr case (a more elaborate discussion of possible strategies for attacking the Kerr problem can be found in [6]). The framework of the current code, i.e., numerical evolution in 1+1D is not directly applicable in Kerr spacetime, because the perturbation equations in Kerr cannot be separated into harmonics in the time domain. It is possible to tackle the field equations in $2+1 \mathrm{D}$ (i.e, 
separating the perturbation into azimuthal $m$-modes only) or in full $3+1 \mathrm{D}$, and there has been considerable progress in that direction in the past two years - although work so far has been restricted to the toy problem of a scalar field in Schwarzschild geometry [11, 55, 59, 60. Schemes for regularizing the SF directly in 2+1D or 3+1D have been proposed and recently implemented [55, 60, 61]. Alternatively, one may attempt to tackle the field equations in 1+1D by properly accounting for the coupling between different $l$-harmonics, and a similar strategy may be applicable in the frequency domain too. A first calculation of the SF in the Kerr case (using the frequency-domain approach) - for a scalar charge in a circular equatorial orbit - will be presented in a forthcoming paper [62].

\section{ACKNOWLEDGEMENTS}

We would like to thank Ryuichi Fujita for providing us (on request) with the accurate flux data shown in Table $\mathrm{V}$ and for permitting us to include these data here. We are grateful to Thibault Damour for useful comments on the manuscript. LB acknowledges support from STFC through Grant No. PP/E001025/1, and wishes to thank Misao Sasaki and the Yukawa Institute for Theoretical Physics in Kyoto (where part of this work took place) for their hospitality. NS acknowledges support from Monbukagaku-sho Grant-in-Aid for the global COE program "The Next Generation of Physics, Spun from Universality and Emergence".

\section{Appendix A: Algebraic reconstruction of the metric components in the Lorenz gauge}

We prescribe here the construction of the various components of the (trace-reversed) Lorenz-gauge metric perturbation $\bar{h}_{\alpha \beta}$ in terms of the 10 time-radial scalar-like functions $\bar{h}^{(i) l m}$ introduced in Eq. (2.19). In the following $Y^{l m}=Y^{l m}(\theta, \varphi)$ are the standard spherical harmonics, $f=1-2 M / r$, and for brevity we suppress the multipolar indices $l, m$ in $\bar{h}^{(i) l m}$. Recall that the functions $\bar{h}^{(i) l m}$ - our basic numerical evolution variables - are obtained by solving the coupled set (2.20).

The metric perturbation is reconstructed through

$$
h_{\alpha \beta}=\frac{\mu}{2 r} \sum_{l=0}^{\infty} \sum_{m=-l}^{l} h_{\alpha \beta}^{l m},
$$

where the various Schwarzschild components are given by

$$
\begin{aligned}
& h_{t t}^{l m}=\left(\bar{h}^{(1)}+f \bar{h}^{(6)}\right) Y^{l m}, \\
& h_{t r}^{l m}=f^{-1} \bar{h}^{(2)} Y^{l m}, \\
& h_{r r}^{l m}=f^{-2}\left(\bar{h}^{(1)}-f \bar{h}^{(6)}\right) Y^{l m}, \\
& h_{t \theta}^{l m}=r\left(\bar{h}^{(4)} Y_{\mathrm{V} 1}^{l m}+\bar{h}^{(8)} Y_{\mathrm{V} 2}^{l m}\right), \\
& h_{t \varphi}^{l m}=r \sin \theta\left(\bar{h}^{(4)} Y_{\mathrm{V} 2}^{l m}-\bar{h}^{(8)} Y_{\mathrm{V} 1}^{l m}\right), \\
& h_{r \theta}^{l m}=r f^{-1}\left(\bar{h}^{(5)} Y_{\mathrm{V} 1}^{l m}+\bar{h}^{(9)} Y_{\mathrm{V} 2}^{l m}\right), \\
& h_{r \varphi}^{l m}=r f^{-1} \sin \theta\left(\bar{h}^{(5)} Y_{\mathrm{V} 2}^{l m}-\bar{h}^{(9)} Y_{\mathrm{V} 1}^{l m}\right), \\
& h_{\theta \theta}^{l m}=r^{2}\left(\bar{h}^{(3)} Y^{l m}+\bar{h}^{(7)} Y_{\mathrm{T} 1}^{l m}+\bar{h}^{(10)} Y_{\mathrm{T} 2}^{l m}\right), \\
& h_{\theta \varphi}^{l m}=r^{2} \sin \theta\left(\bar{h}^{(7)} Y_{\mathrm{T} 2}^{l m}-\bar{h}^{(10)} Y_{\mathrm{T} 1}^{l m}\right), \\
& h_{\varphi \varphi}^{l m}=r^{2} \sin ^{2} \theta\left(\bar{h}^{(3)} Y^{l m}-\bar{h}^{(7)} Y_{\mathrm{T} 1}^{l m}-\bar{h}^{(10)} Y_{\mathrm{T} 2}^{l m}\right) .
\end{aligned}
$$


The angular functions appearing in these relations are defined as

$$
\begin{aligned}
Y_{\mathrm{V} 1}^{l m}(\theta, \varphi) & \equiv \frac{1}{l(l+1)} Y_{, \theta}^{l m} \quad(\text { for } l>0), \\
Y_{\mathrm{V} 2}^{l m}(\theta, \varphi) & \equiv \frac{1}{l(l+1)} \sin ^{-1} \theta Y_{, \varphi}^{l m} \quad(\text { for } l>0), \\
Y_{\mathrm{T} 1}^{l m}(\theta, \varphi) & \equiv \frac{(l-2) !}{(l+2) !}\left[\sin \theta\left(\sin ^{-1} \theta Y_{, \theta}^{l m}\right)_{, \theta}-\sin ^{-2} \theta Y_{, \varphi \varphi}^{l m}\right] \quad(\text { for } l>1), \\
Y_{\mathrm{T} 2}^{l m}(\theta, \varphi) & \equiv \frac{2(l-2) !}{(l+2) !}\left(\sin ^{-1} \theta Y_{, \varphi}^{l m}\right)_{, \theta} \quad(\text { for } l>1) .
\end{aligned}
$$

We note that for $l=0,1$ we have $h^{(7,10)}=0$ identically, and that for $l=0$ we have additionally $h^{(4,5,8,9)}=0$.

\section{Appendix B: Field equations and gauge conditions for the perturbation functions $\bar{h}^{(i) l m}(r, t)$}

We give here explicit expressions for the various terms appearing in our 1+1D field equation (2.20). In what follows $f=1-2 M / r, f^{\prime}=2 M / r^{2}, r_{*}$ is the standard tortoise radial coordinate defined through $d r / d r_{*}=f(r)$, and $v=t+r_{*}$. We also denote $\lambda=(l+2)(l-1)$.

The terms $\mathcal{M}_{(j)}^{(i) l} \bar{h}^{(j) l m}$ in Eq. (2.20) read

$$
\begin{aligned}
& \mathcal{M}_{(j)}^{(1)} \bar{h}^{(j)}=\frac{\partial}{\partial r_{*}}\left(\frac{1}{2} f f^{\prime} \bar{h}^{(3)}\right)+\frac{(r-4 M) f}{2 r^{3}}\left(\bar{h}^{(1)}-\bar{h}^{(5)}\right)-\frac{\left(r^{2}-10 M r+20 M^{2}\right) f}{2 r^{4}} \bar{h}^{(3)}-\frac{(r-6 M) f^{2}}{2 r^{3}} \bar{h}^{(6)} \\
& \mathcal{M}_{(j)}^{(2)} \bar{h}^{(j)}=\frac{\partial}{\partial r_{*}}\left(\frac{1}{2} f f^{\prime} \bar{h}^{(3)}\right)+\frac{\partial}{\partial v}\left[f^{\prime}\left(\bar{h}^{(2)}-\bar{h}^{(1)}\right)\right]-\frac{3 M f}{r^{3}} \bar{h}^{(1)}+\frac{(r+2 M) f}{2 r^{3}} \bar{h}^{(2)}+\frac{(3 r-8 M) M f}{r^{4}} \bar{h}^{(3)} \\
& -\frac{f^{2}}{2 r^{2}} \bar{h}^{(4)}+\frac{f f^{\prime}}{2 r} \bar{h}^{(5)}+\frac{f^{2} f^{\prime}}{r} \bar{h}^{(6)} \\
& \mathcal{M}_{(j)}^{(3)} \bar{h}^{(j)}=-\frac{f}{2 r^{2}}\left[\bar{h}^{(1)}-\bar{h}^{(5)}-\left(1-\frac{4 M}{r}\right)\left(\bar{h}^{(3)}+\bar{h}^{(6)}\right)\right] \\
& \mathcal{M}_{(j)}^{(4)} \bar{h}^{(j)}=\frac{\partial}{\partial v}\left[\frac{f^{\prime}}{2}\left(\bar{h}^{(4)}-\bar{h}^{(5)}\right)\right]-\frac{l(l+1) f}{2 r^{2}} \bar{h}^{(2)}-\frac{M f}{2 r^{3}} \bar{h}^{(4)}-\frac{2 M f}{r^{3}} \bar{h}^{(5)}-\frac{l(l+1) f f^{\prime}}{4 r} \bar{h}^{(6)}+\frac{f f^{\prime}}{4 r} \bar{h}^{(7)} \\
& \mathcal{M}_{(j)}^{(5)} \bar{h}^{(j)}=\frac{f}{r^{2}}\left[\left(1-\frac{9 M}{2 r}\right) \bar{h}^{(5)}-\frac{1}{2} l(l+1)\left(\bar{h}^{(1)}-f \bar{h}^{(3)}\right)+\frac{1}{2}\left(1-\frac{3 M}{r}\right)\left(l(l+1) \bar{h}^{(6)}-\bar{h}^{(7)}\right)\right] \\
& \mathcal{M}_{(j)}^{(6)} \bar{h}^{(j)}=-\frac{f}{2 r^{2}}\left[\bar{h}^{(1)}-\bar{h}^{(5)}-\left(1-\frac{4 M}{r}\right)\left(\bar{h}^{(3)}+\bar{h}^{(6)}\right)\right] \\
& \mathcal{M}_{(j)}^{(7)} \bar{h}^{(j)}=-\frac{f}{2 r^{2}}\left(\bar{h}^{(7)}+\lambda \bar{h}^{(5)}\right) \\
& \mathcal{M}_{(j)}^{(8)} \bar{h}^{(j)}=\frac{\partial}{\partial v}\left[\frac{f^{\prime}}{2}\left(\bar{h}^{(8)}-\bar{h}^{(9)}\right)\right]-\frac{M f}{2 r^{3}} \bar{h}^{(8)}-\frac{2 M f}{r^{3}} \bar{h}^{(9)}+\frac{M f}{2 r^{3}} \bar{h}^{(10)} \\
& \mathcal{M}_{(j)}^{(9)} \bar{h}^{(j)}=\frac{f}{r^{2}}\left(1-\frac{9 M}{2 r}\right) \bar{h}^{(9)}-\frac{f}{2 r^{2}}\left(1-\frac{3 M}{r}\right) \bar{h}^{(10)}
\end{aligned}
$$




$$
\mathcal{M}_{(j)}^{(10)} \bar{h}^{(j)}=-\frac{f}{2 r^{2}}\left(\bar{h}^{(10)}+\lambda \bar{h}^{(9)}\right)
$$

These expression are the same as those given in Appendix A of paper I, although we write them here in a slightly different form, more amenable to discretization in $v, u$ coordinates.

The various source terms $S^{(i) l m}$ in Eq. (2.20) are given (referring to Sec. II A for notation) by

$$
\begin{aligned}
S_{l m}^{(1)} & =\frac{4 \pi f_{\mathrm{p}}^{2}}{\mathcal{E} r_{\mathrm{p}}^{3}}\left(2 \mathcal{E}^{2} r_{\mathrm{p}}^{2}-f_{\mathrm{p}} r_{\mathrm{p}}^{2}-\mathcal{L}^{2} f_{\mathrm{p}}\right) Y_{l m}^{*}\left(\pi / 2, \varphi_{\mathrm{p}}\right), \\
S_{l m}^{(2)} & =-\frac{8 \pi f_{\mathrm{p}}^{2}}{r_{\mathrm{p}}} u^{r} Y_{l m}^{*}\left(\pi / 2, \varphi_{\mathrm{p}}\right), \\
S_{l m}^{(3)} & =\frac{4 \pi}{\mathcal{E} r_{\mathrm{p}}^{3}} f_{\mathrm{p}}^{2}\left(r_{\mathrm{p}}^{2}+\mathcal{L}^{2}\right) Y_{l m}^{*}\left(\pi / 2, \varphi_{\mathrm{p}}\right), \\
S_{l m}^{(4)} & =\frac{8 \pi i m f_{\mathrm{p}}^{2} \mathcal{L}}{r_{\mathrm{p}}^{2}} Y_{l m}^{*}\left(\pi / 2, \varphi_{\mathrm{p}}\right), \\
S_{l m}^{(5)} & =-\frac{8 \pi i m f_{\mathrm{p}}^{2} u^{r} \mathcal{L}}{r_{\mathrm{p}}^{2} \mathcal{E}} Y_{l m}^{*}\left(\pi / 2, \varphi_{\mathrm{p}}\right), \\
S_{l m}^{(6)} & =\frac{4 \pi f_{\mathrm{p}}^{2} \mathcal{L}^{2}}{r_{\mathrm{p}}^{3} \mathcal{E}} Y_{l m}^{*}\left(\pi / 2, \varphi_{\mathrm{p}}\right), \\
S_{l m}^{(7)} & =\left[l(l+1)-2 m^{2}\right] S_{l m}^{(6)}, \\
S_{l m}^{(8)} & =\frac{8 \pi f_{\mathrm{p}}^{2} \mathcal{L}}{r_{\mathrm{p}}^{2}} Y_{l m, \theta}^{*}\left(\pi / 2, \varphi_{\mathrm{p}}\right), \\
S_{l m}^{(9)} & =-\frac{8 \pi f_{\mathrm{p}}^{2} u^{r} \mathcal{L}}{r_{\mathrm{p}}^{2} \mathcal{E}} Y_{l m, \theta}^{*}\left(\pi / 2, \varphi_{\mathrm{p}}\right), \\
S_{l m}^{(10)} & =\frac{8 \pi i m f_{\mathrm{p}}^{2} \mathcal{L}^{2}}{r_{\mathrm{p}}^{3} \mathcal{E}} Y_{l m, \theta}^{*}\left(\pi / 2, \varphi_{\mathrm{p}}\right) .
\end{aligned}
$$

The 1+1D field equations (2.20) are supplemented by 4 elliptic "constraints" stemming from the Lorenz-gauge conditions (2.15). These constraint equations read

$$
\begin{gathered}
-\bar{h}_{, t}^{(1)}+f\left(-\bar{h}_{, t}^{(3)}+\bar{h}_{, r}^{(2)}+\frac{\bar{h}^{(2)}-\bar{h}^{(4)}}{r}\right)=0, \\
\bar{h}_{, t}^{(2)}-f \bar{h}_{, r}^{(1)}+f^{2} \bar{h}_{, r}^{(3)}-\frac{f}{r}\left(\bar{h}^{(1)}-\bar{h}^{(5)}-f \bar{h}^{(3)}-2 f \bar{h}^{(6)}\right)=0, \\
\bar{h}_{, t}^{(4)}-\frac{f}{r}\left(r \bar{h}_{, r}^{(5)}+2 \bar{h}^{(5)}+l(l+1) \bar{h}^{(6)}-\bar{h}^{(7)}\right)=0, \\
\bar{h}_{, t}^{(8)}-\frac{f}{r}\left(r \bar{h}_{, r}^{(9)}+2 \bar{h}^{(9)}-\bar{h}^{(10)}\right)=0 .
\end{gathered}
$$




\section{Appendix C: Construction of the full-force spherical-harmonic modes}

Following are the explicit values of the functions $\mathcal{F}_{(n)}^{\alpha l m}$ appearing in Eq. (2.25):

$$
\begin{aligned}
& \mathcal{F}_{(-3)}^{\alpha l m}=\zeta_{(+3)}^{l m} f_{6 \pm}^{\alpha l m}+\xi_{(+3)}^{l m} f_{7 \pm}^{\alpha l m}, \\
& \mathcal{F}_{(-2)}^{\alpha l m}=\alpha_{(+2)}^{l m} f_{1 \pm}^{\alpha l m}+\beta_{(+2)}^{l m} f_{2 \pm}^{\alpha l m}+\gamma_{(+2)}^{l m} f_{3 \pm}^{\alpha l m}, \\
& \mathcal{F}_{(-1)}^{\alpha l m}=\epsilon_{(+1)}^{l m} f_{4 \pm}^{\alpha l m}+\delta_{(+1)}^{l m} f_{5 \pm}^{\alpha l m}+\zeta_{(+1)}^{l m} f_{6 \pm}^{\alpha l m}+\xi_{(+1)}^{l m} f_{7 \pm}^{\alpha l m} \\
& \mathcal{F}_{(0)}^{\alpha l m}=f_{0 \pm}^{\alpha l m}+\alpha_{(0)}^{\alpha l m} f_{1 \pm}^{\alpha l m}+\beta_{(0)}^{l l m} f_{2 \pm}^{\alpha l m}+\gamma_{(0)}^{l m} f_{3 \pm}^{\alpha l m}, \\
& \mathcal{F}_{(+1)}^{\alpha l m}=\epsilon_{(-1)}^{l m} f_{4 \pm}^{\alpha l m}+\delta_{(-1)}^{l m} f_{5 \pm}^{\alpha l m}+\zeta_{(-1)}^{l m} f_{6 \pm}^{\alpha l m}+\xi_{(-1)}^{l m} f_{7 \pm}^{\alpha l m} \\
& \mathcal{F}_{(+2)}^{\alpha l m}=\alpha_{(-2)}^{l m} f_{1 \pm}^{\alpha l m}+\beta_{(-2)}^{l m} f_{2 \pm}^{\alpha l m}+\gamma_{(-2)}^{l m} f_{3 \pm}^{\alpha l m} \\
& \mathcal{F}_{(+3)}^{\alpha l m}=\zeta_{(-3)}^{l m} f_{6 \pm}^{\alpha l m}+\xi_{(-3)}^{l m} f_{7 \pm}^{\alpha l m}
\end{aligned}
$$

The various functions $f_{n \pm}^{\alpha l m}$ are those appearing in Eq. (2.24), and below we give these functions explicitly for $\alpha=t, r$ (the values for $\varphi, \theta$ are not needed in this work). The values of the various $l, m$-dependent coefficients $\alpha, \beta, \gamma, \epsilon, \zeta, \xi$ in the above expressions are given in Eqs. (D3)-(D9) of Appendix D In what follows we use the notation $\hat{\mathcal{L}} \equiv \mathcal{L} / r_{\mathrm{p}}$, and for brevity we suppress the indices $l, m$ as well as the subscript \pm ; it is to be understood that the $t$ and $r$ derivatives in the following expressions are taken from either "outside" or "inside" the orbit, yielding, in general, two different one-sided values which correspond to the suppressed + or - subscripts.

For the $t$ component we have-

$$
\begin{aligned}
& f_{0}^{t}=\frac{\mathcal{E}}{4 f_{\mathrm{p}}^{4}}\left[\left(u^{r}\right)^{3}+\left(\mathcal{E}^{2} \frac{r_{\mathrm{p}}+4 M}{r_{\mathrm{p}}}-2 f_{\mathrm{p}}\right) u^{r}-i m f_{\mathrm{p}} \hat{\mathcal{L}}\left(\left(u^{r}\right)^{2}+\mathcal{E}^{2}-2 f_{\mathrm{p}}\right)\right] \bar{h}^{(1)} \\
& -\frac{r_{\mathrm{p}}}{4 f_{\mathrm{p}}^{4}}\left[\left(\mathcal{E}^{2}+f_{\mathrm{p}}\right)\left(u^{r}\right)^{2}+\mathcal{E}^{2}\left(\mathcal{E}^{2}-f_{\mathrm{p}}\right)\right] \bar{h}_{, t}^{(1)}-\frac{r_{\mathrm{p}}}{4 f_{\mathrm{p}}^{4}} u^{r} \mathcal{E}\left[\left(u^{r}\right)^{2}+\mathcal{E}^{2}-2 f_{\mathrm{p}}\right] \bar{h}_{, r_{*}}^{(1)} \\
& +\frac{1}{2 f_{\mathrm{p}}^{4}}\left[\left(u^{r}\right)^{2}\left(\mathcal{E}^{2} \frac{r_{\mathrm{p}}+M}{r_{\mathrm{p}}}-f_{\mathrm{p}} \frac{r_{\mathrm{p}}-M}{r_{\mathrm{p}}}\right)+\frac{M}{r_{\mathrm{p}}} \mathcal{E}^{2}\left(\mathcal{E}^{2}-f_{\mathrm{p}}\right)-i m f_{\mathrm{p}} u^{r} \hat{\mathcal{L}}\left(\mathcal{E}^{2}-f_{\mathrm{p}}\right)\right] \bar{h}^{(2)} \\
& -\frac{r_{\mathrm{p}} u^{r}}{2 f_{\mathrm{p}}^{4}}\left[\mathcal{E}^{3} \bar{h}_{, t}^{(2)}+u^{r}\left(\mathcal{E}^{2}-f_{\mathrm{p}}\right) \bar{h}_{, r_{*}}^{(2)}\right]+\frac{\mathcal{E} \hat{\mathcal{L}}^{2}}{4 f_{\mathrm{p}}}\left(u^{r}-i m \hat{\mathcal{L}}\right) \bar{h}^{(3)}-\frac{r_{\mathrm{p}} \hat{\mathcal{L}}^{2}}{4 f_{\mathrm{p}}^{2}}\left[\left(\mathcal{E}^{2}+f_{\mathrm{p}}\right) \bar{h}_{, t}^{(3)}+u^{r} \overline{\mathcal{L}}_{{ }_{r_{*}}}^{(3)}\right] \\
& +\frac{m}{2 l(l+1) f_{\mathrm{p}}^{3}} \hat{\mathcal{L}}\left[2 i u^{r}\left(\mathcal{E}^{2} \frac{r_{\mathrm{p}}-M}{r_{\mathrm{p}}}-f_{\mathrm{p}}^{2}\right)+m f_{\mathrm{p}} \hat{\mathcal{L}}\left(\mathcal{E}^{2}-f_{\mathrm{p}}\right)\right] \bar{h}^{(4)} \\
& -\frac{i m r_{\mathrm{p}} \hat{\mathcal{L}}}{2 l(l+1) f_{\mathrm{p}}^{3}}\left[\mathcal{E}^{3} \bar{h}_{, t}^{(4)}+u^{r}\left(\mathcal{E}^{2}-f_{\mathrm{p}}\right) \bar{h}_{, r_{*}}^{(4)}\right]+\frac{m \mathcal{E} \hat{\mathcal{L}}}{2 l(l+1) f_{\mathrm{p}}^{3}}\left[i\left(u^{r}\right)^{2}\left(2-\frac{3 M}{r_{\mathrm{p}}}\right)+\frac{i M}{r_{\mathrm{p}}} \mathcal{E}^{2}+m f_{\mathrm{p}} u^{r} \hat{\mathcal{L}}\right] \bar{h}^{(5)} \\
& -\frac{i m r_{\mathrm{p}} u^{r} \hat{\mathcal{L}}}{2 l(l+1) f_{\mathrm{p}}^{3}}\left[\left(\mathcal{E}^{2}+f_{\mathrm{p}}\right) \bar{h}_{, t}^{(5)}+u^{r} \mathcal{E} \bar{h}_{, r_{*}}^{(5)}\right]+\frac{\mathcal{E}}{4 f_{\mathrm{p}}}\left(-u^{r}+i m \hat{\mathcal{L}}\right) \bar{h}^{(6)}+\frac{r_{\mathrm{p}}}{4 f_{\mathrm{p}}^{2}}\left[\left(\mathcal{E}^{2}-f_{\mathrm{p}}\right) \bar{h}_{, t}^{(6)}+u^{r} \mathcal{E} \bar{h}_{, r_{*}}^{(6)}\right] \\
& -\frac{m \mathcal{E} \hat{\mathcal{L}}^{2}}{4 l(l+1) \lambda f_{\mathrm{p}}}\left[3 m u^{r}-i \hat{\mathcal{L}}\left(4+m^{2}\right)\right] \bar{h}^{(7)}+\frac{m^{2} r_{\mathrm{p}} \hat{\mathcal{L}}^{2}}{4 l(l+1) \lambda f_{\mathrm{p}}^{2}}\left[\left(\mathcal{E}^{2}+f_{\mathrm{p}}\right) \bar{h}_{, t}^{(7)}+u^{r} \mathcal{E} \bar{h}_{, r_{*}}^{(7)}\right], \\
& f_{1}^{t}=-\frac{u^{r} \mathcal{E} \hat{\mathcal{L}}^{2}}{2 f_{\mathrm{p}}^{2}} \bar{h}^{(1)}-\frac{\hat{\mathcal{L}}^{2}}{2 f_{\mathrm{p}}^{2}}\left(\mathcal{E}^{2}-f_{\mathrm{p}}\right) \bar{h}^{(2)}+\frac{u^{r} \mathcal{E} \hat{\mathcal{L}}^{2}}{2 f_{\mathrm{p}}} \bar{h}^{(3)}-\frac{i m \mathcal{E} \hat{\mathcal{L}}^{3}}{2 l(l+1) f_{\mathrm{p}}} \bar{h}^{(5)} \\
& +\frac{\mathcal{E} \hat{\mathcal{L}}^{2}}{4 f_{\mathrm{p}}}\left(3 u^{r}-i m \hat{\mathcal{L}}\right) \bar{h}^{(6)}-\frac{r_{\mathrm{p}}}{4 f_{\mathrm{p}}^{2}} \hat{\mathcal{L}}^{2}\left(\mathcal{E}^{2}+f_{\mathrm{p}}\right) \bar{h}_{, t}^{(6)}-\frac{r_{\mathrm{p}}}{4 f_{\mathrm{p}}^{2}} u^{r} \mathcal{E} \hat{\mathcal{L}}^{2} \bar{h}_{, r_{*}}^{(6)}-\frac{i m \mathcal{E} \hat{\mathcal{L}}^{3}}{l(l+1) \lambda f_{\mathrm{p}}} \bar{h}^{(7)}, \\
& f_{2}^{t}=-\frac{\hat{\mathcal{L}}^{2}\left(\mathcal{E}^{2}-f_{\mathrm{p}}\right)}{2 l(l+1) f_{\mathrm{p}}^{2}} \bar{h}^{(4)}-\frac{u^{r} \mathcal{E} \hat{\mathcal{L}}^{2}}{2 l(l+1) f_{\mathrm{p}}^{2}} \bar{h}^{(5)} \\
& +\frac{\hat{\mathcal{L}}^{2}}{4 l(l+1) \lambda f_{\mathrm{p}}^{2}}\left[f_{\mathrm{p}} \mathcal{E}\left(3 u^{r}-5 i m \hat{\mathcal{L}}\right) \bar{h}^{(7)}-r_{\mathrm{p}}\left(\mathcal{E}^{2}+f_{\mathrm{p}}\right) \bar{h}_{, t}^{(7)}-r_{\mathrm{p}} u^{r} \mathcal{E} \bar{h}_{, r_{*}}^{(7)}\right],
\end{aligned}
$$




$$
\begin{aligned}
& f_{3}^{t}=\frac{\hat{\mathcal{L}}^{2}}{4 l(l+1) \lambda f_{\mathrm{p}}^{2}}\left[-f_{\mathrm{p}} \mathcal{E}\left(3 u^{r}-i m \hat{\mathcal{L}}\right) \bar{h}^{(7)}+r_{\mathrm{p}}\left(\mathcal{E}^{2}+f_{\mathrm{p}}\right) \bar{h}_{, t}^{(7)}+r_{\mathrm{p}} u^{r} \mathcal{E} \bar{h}_{, r_{*}}^{(7)}\right] \\
& f_{4}^{t}=-\frac{i m \hat{\mathcal{L}}^{2}}{2 l(l+1) f_{\mathrm{p}}^{2}}\left[\left(\mathcal{E}^{2}-f_{\mathrm{p}}\right) \bar{h}^{(8)}+u^{r} \mathcal{E} \bar{h}^{(9)}\right] \\
& +\frac{i m \hat{\mathcal{L}}^{2}}{2 l(l+1) \lambda f_{\mathrm{p}}^{2}}\left[f_{\mathrm{p}} \mathcal{E}\left(3 u^{r}-2 i m \hat{\mathcal{L}}\right) \bar{h}^{(10)}-r_{\mathrm{p}}\left(\mathcal{E}^{2}+f_{\mathrm{p}}\right) \bar{h}_{, t}^{(10)}-r_{\mathrm{p}} u^{r} \mathcal{E} \bar{h}_{, r_{*}}^{(10)}\right], \\
& f_{5}^{t}=-\frac{u^{r} \hat{\mathcal{L}}}{l(l+1) f_{\mathrm{p}}^{3}}\left(\mathcal{E}^{2} \frac{r_{\mathrm{p}}-M}{r_{\mathrm{p}}}-f_{\mathrm{p}}^{2}\right) \bar{h}^{(8)}+\frac{r_{\mathrm{p}} \mathcal{E}^{3} \hat{\mathcal{L}}}{2 l(l+1) f_{\mathrm{p}}^{3}} \bar{h}_{, t}^{(8)}+\frac{r_{\mathrm{p}} u^{r} \hat{\mathcal{L}}}{2 l(l+1) f_{\mathrm{p}}^{3}}\left(\mathcal{E}^{2}-f_{\mathrm{p}}\right) \bar{h}_{, r_{*}}^{(8)} \\
& -\frac{\mathcal{E} \hat{\mathcal{L}}}{2 l(l+1) f_{\mathrm{p}}^{3}}\left[\left(u^{r}\right)^{2} \frac{2 r_{\mathrm{p}}-3 M}{r_{\mathrm{p}}}+\frac{M}{r_{\mathrm{p}}} \mathcal{E}^{2}\right] \bar{h}^{(9)}+\frac{r_{\mathrm{p}} u^{r} \hat{\mathcal{L}}}{2 l(l+1) f_{\mathrm{p}}^{3}}\left(\mathcal{E}^{2}+f_{\mathrm{p}}\right) \bar{h}_{, t}^{(9)}+\frac{r_{\mathrm{p}}\left(u^{r}\right)^{2} \mathcal{E} \hat{\mathcal{L}}}{2 l(l+1) f_{\mathrm{p}}^{3}} \bar{h}_{, r_{*}}^{(9)} \\
& +\frac{\left(m^{2}-1\right) \mathcal{E} \hat{\mathcal{L}}^{3}}{2 l(l+1) \lambda f_{\mathrm{p}}} \bar{h}^{(10)}
\end{aligned}
$$

For the $r$ component we have-

$$
\begin{aligned}
& f_{0}^{r}=\frac{1}{4 f_{\mathrm{p}}^{3}}\left[\left(u^{r}\right)^{4}-i m f_{\mathrm{p}}\left(u^{r}\right)^{3} \hat{\mathcal{L}}+\left(u^{r}\right)^{2}\left(\frac{r_{\mathrm{p}}+4 M}{r_{\mathrm{p}}} \mathcal{E}^{2}+f_{\mathrm{p}}\right)-i m u^{r} f_{\mathrm{p}} \hat{\mathcal{L}}\left(\mathcal{E}^{2}+2 f_{\mathrm{p}}\right)-f_{\mathrm{p}} \mathcal{E}^{2}\left(1-\frac{4 M}{r_{\mathrm{p}}}\right)\right] \bar{h}^{(1)} \\
& -\frac{r_{\mathrm{p}} u^{r} \mathcal{E}}{4 f_{\mathrm{p}}^{3}}\left[\left(u^{r}\right)^{2}+\mathcal{E}^{2}+2 f_{\mathrm{p}}\right] \bar{h}_{, t}^{(1)}-\frac{r_{\mathrm{p}}}{4 f_{\mathrm{p}}^{3}}\left[\left(u^{r}\right)^{4}+\left(u^{r}\right)^{2}\left(\mathcal{E}^{2}+f_{\mathrm{p}}\right)-f_{\mathrm{p}} \mathcal{E}^{2}\right] \bar{h}_{, r_{*}}^{(1)} \\
& +\frac{\mathcal{E}}{2 f_{\mathrm{p}}^{3}}\left[\left(u^{r}\right)^{3}\left(1+\frac{M}{r_{\mathrm{p}}}\right)-i m f_{\mathrm{p}}\left(u^{r}\right)^{2} \hat{\mathcal{L}}+\frac{M}{r_{\mathrm{p}}} u^{r}\left(\mathcal{E}^{2}+2 f_{\mathrm{p}}\right)-i m f_{\mathrm{p}}^{2} \hat{\mathcal{L}}\right] \bar{h}^{(2)} \\
& -\frac{r_{\mathrm{p}} \mathcal{E}^{2}}{2 f_{\mathrm{p}}^{3}}\left[\left(u^{r}\right)^{2}+f_{\mathrm{p}}\right] \bar{h}_{, t}^{(2)}-\frac{r_{\mathrm{p}}\left(u^{r}\right)^{3} \mathcal{E}_{\bar{h}^{(2)}}}{2 f_{\mathrm{p}}^{3}}{ }_{, r_{*}} \\
& +\frac{1}{4 f_{\mathrm{p}}}\left[-\left(u^{r}\right)^{4}+i m \hat{\mathcal{L}}\left(u^{r}\right)^{3}+\mathcal{E}^{2}\left(u^{r}\right)^{2}-\left(i m u^{r} \hat{\mathcal{L}}+f_{\mathrm{p}}\right)\left(\mathcal{E}^{2}-f_{\mathrm{p}}\right)\right] \bar{h}^{(3)} \\
& +\frac{r_{\mathrm{p}} u^{r} \mathcal{E}}{4 f_{\mathrm{p}}^{2}}\left[\left(u^{r}\right)^{2}-\mathcal{E}^{2}+f_{\mathrm{p}}\right] \bar{h}_{, t}^{(3)}+\frac{r_{\mathrm{p}}}{4 f_{\mathrm{p}}^{2}}\left[\left(u^{r}\right)^{4}-\mathcal{E}^{2}\left(u^{r}\right)^{2}+f_{\mathrm{p}}\left(\mathcal{E}^{2}-f_{\mathrm{p}}\right)\right] \bar{h}_{, r_{*}}^{(3)}
\end{aligned}
$$

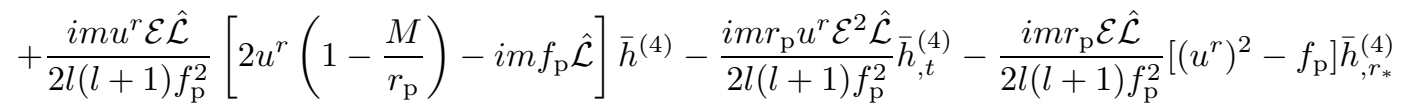

$$
\begin{aligned}
& +\frac{i m \hat{\mathcal{L}}}{2 l(l+1) f_{\mathrm{p}}^{2}}\left[\left(u^{r}\right)^{3}\left(2-\frac{3 M}{r_{\mathrm{p}}}\right)-i m f_{\mathrm{p}}\left(u^{r}\right)^{2} \hat{\mathcal{L}}+u^{r}\left(\frac{M}{r_{\mathrm{p}}} \mathcal{E}^{2}+2 f_{\mathrm{p}}^{2}\right)-i m f_{\mathrm{p}}^{2} \hat{\mathcal{L}}\right] \bar{h}^{(5)} \\
& -\frac{i m r_{\mathrm{p}} \mathcal{E} \hat{\mathcal{L}}}{2 l(l+1) f_{\mathrm{p}}^{2}}\left[\left(u^{r}\right)^{2}+f_{\mathrm{p}}\right] \bar{h}_{, t}^{(5)}-\frac{i m r_{\mathrm{p}}\left(u^{r}\right)^{3} \hat{\mathcal{L}}_{\overline{\mathcal{L}}}}{2 l(l+1) f_{\mathrm{p}}^{2}}{ }_{, r_{*}}^{(5)}-\frac{1}{4}\left[\left(u^{r}\right)^{2}-i m u^{r} \hat{\mathcal{L}}+f_{\mathrm{p}}\right] \bar{h}^{(6)}+\frac{r_{\mathrm{p}} u^{r} \mathcal{E}}{4 f_{\mathrm{p}}} \bar{h}_{, t}^{(6)} \\
& +\frac{r_{\mathrm{p}}}{4 f_{\mathrm{p}}}\left[\left(u^{r}\right)^{2}+f_{\mathrm{p}}\right] \bar{h}_{, r_{*}}^{(6)}+\frac{m \hat{\mathcal{L}}^{2}}{4 l(l+1) \lambda}\left[-3 m\left(u^{r}\right)^{2}+i u^{r} \hat{\mathcal{L}}\left(4+m^{2}\right)-m f_{\mathrm{p}}\right] \bar{h}^{(7)}+\frac{m^{2} r_{\mathrm{p}} u^{r} \mathcal{E} \hat{\mathcal{L}}^{2}}{4 l(l+1) \lambda f_{\mathrm{p}}} \bar{h}_{, t}^{(7)} \\
& +\frac{m^{2} r_{\mathrm{p}} \hat{\mathcal{L}}^{2}}{4 l(l+1) \lambda f_{\mathrm{p}}}\left[\left(u^{r}\right)^{2}-f_{\mathrm{p}}\right] \bar{h}_{, r_{*}}^{(7)}
\end{aligned}
$$




$$
\begin{aligned}
& f_{1}^{r}=-\frac{\hat{\mathcal{L}}^{2}}{2 f_{\mathrm{p}}}\left[\left(u^{r}\right)^{2}+f_{\mathrm{p}}\right] \bar{h}^{(1)}-\frac{u^{r} \mathcal{E} \hat{\mathcal{L}}^{2}}{2 f_{\mathrm{p}}} \bar{h}^{(2)}+\frac{\hat{\mathcal{L}}^{2}}{2}\left[\left(u^{r}\right)^{2}+f_{\mathrm{p}}\right] \bar{h}^{(3)}-\frac{i m u^{r} \hat{\mathcal{L}}^{3}}{2 l(l+1)} \bar{h}^{(5)} \\
& +\frac{\hat{\mathcal{L}}^{2}}{4}\left[3\left(u^{r}\right)^{2}-i m u^{r} \hat{\mathcal{L}}+f_{\mathrm{p}}\right] \bar{h}^{(6)}-\frac{r_{\mathrm{p}} u^{r} \mathcal{E} \hat{\mathcal{L}}^{2}}{4 f_{\mathrm{p}}} \bar{h}_{, t}^{(6)}-\frac{r_{\mathrm{p}} \hat{\mathcal{L}}^{2}}{4 f_{\mathrm{p}}}\left[\left(u^{r}\right)^{2}-f_{\mathrm{p}}\right] \bar{h}_{, r_{*}}^{(6)}-\frac{i m u^{r} \hat{\mathcal{L}}^{3}}{l(l+1) \lambda} \bar{h}^{(7)}, \\
& f_{2}^{r}=-\frac{\hat{\mathcal{L}}^{2}}{2 l(l+1) f_{\mathrm{p}}}\left[u^{r} \mathcal{E} \bar{h}^{(4)}+\left(\left(u^{r}\right)^{2}+f_{\mathrm{p}}\right) \bar{h}^{(5)}\right] \\
& +\frac{\hat{\mathcal{L}}^{2}}{4 l(l+1) \lambda f_{\mathrm{p}}}\left[f_{\mathrm{p}}\left(3\left(u^{r}\right)^{2}-5 i m u^{r} \hat{\mathcal{L}}+f_{\mathrm{p}}\right) \bar{h}^{(7)}-r_{\mathrm{p}} u^{r} \overline{\mathcal{E}}_{, t}^{(7)}-r_{\mathrm{p}}\left(\left(u^{r}\right)^{2}-f_{\mathrm{p}}\right) \bar{h}_{, r_{*}}^{(7)}\right], \\
& f_{3}^{r}=\frac{\hat{\mathcal{L}}^{2}}{4 l(l+1) \lambda f_{\mathrm{p}}}\left[-f_{\mathrm{p}}\left(3\left(u^{r}\right)^{2}-i m u^{r} \hat{\mathcal{L}}+f_{\mathrm{p}}\right) \bar{h}^{(7)}+r_{\mathrm{p}} u^{r} \mathcal{E} \bar{h}_{, t}^{(7)}+r_{\mathrm{p}}\left(\left(u^{r}\right)^{2}-f_{\mathrm{p}}\right) \bar{h}_{, r_{*}}^{(7)}\right] \\
& f_{4}^{r}=-\frac{i m \hat{\mathcal{L}}^{2}}{2 l(l+1) f_{\mathrm{p}}}\left[u^{r} \mathcal{E} \bar{h}^{(8)}+\left(\left(u^{r}\right)^{2}+f_{\mathrm{p}}\right) \bar{h}^{(9)}\right] \\
& +\frac{i m \hat{\mathcal{L}}^{2}}{2 l(l+1) \lambda f_{\mathrm{p}}}\left[f_{\mathrm{p}}\left(3\left(u^{r}\right)^{2}-2 i m u^{r} \hat{\mathcal{L}}+f_{\mathrm{p}}\right) \bar{h}^{(10)}-r_{\mathrm{p}} u^{r} \mathcal{E} \bar{h}_{, t}^{(10)}-r_{\mathrm{p}}\left(\left(u^{r}\right)^{2}-f_{\mathrm{p}}\right) \bar{h}_{, r_{*}}^{(10)}\right], \\
& f_{5}^{r}=-\frac{\left(u^{r}\right)^{2} \mathcal{E} \hat{\mathcal{L}}}{l(l+1) f_{\mathrm{p}}^{2}}\left(1-\frac{M}{r_{\mathrm{p}}}\right) \bar{h}^{(8)}+\frac{r_{\mathrm{p}} u^{r} \mathcal{E}^{2} \hat{\mathcal{L}}}{2 l(l+1) f_{\mathrm{p}}^{2}} \bar{h}_{, t}^{(8)}+\frac{r_{\mathrm{p}} \mathcal{E} \hat{\mathcal{L}}}{2 l(l+1) f_{\mathrm{p}}^{2}}\left[\left(u^{r}\right)^{2}-f_{\mathrm{p}}\right] \bar{h}_{, r_{*}}^{(8)} \\
& -\frac{u^{r} \hat{\mathcal{L}}}{2 l(l+1) f_{\mathrm{p}}^{2}}\left[\frac{2 r_{\mathrm{p}}-3 M}{r_{\mathrm{p}}}\left(u^{r}\right)^{2}+\frac{M}{r_{\mathrm{p}}} \mathcal{E}^{2}+2 f_{\mathrm{p}}^{2}\right] \bar{h}^{(9)}+\frac{r_{\mathrm{p}} \mathcal{E} \hat{\mathcal{L}}}{2 l(l+1) f_{\mathrm{p}}^{2}}\left[\left(u^{r}\right)^{2}+f_{\mathrm{p}}\right] \bar{h}_{, t}^{(9)} \\
& +\frac{r_{\mathrm{p}}\left(u^{r}\right)^{3} \hat{\mathcal{L}}}{2 l(l+1) f_{\mathrm{p}}^{2}} \bar{h}_{, r_{*}}^{(9)}+\frac{\left(m^{2}-1\right) u^{r} \hat{\mathcal{L}}^{3}}{2 l(l+1) \lambda} \bar{h}^{(10)}, \\
& f_{6}^{r}=\frac{u^{r} \hat{\mathcal{L}}^{3}}{2 l(l+1) \lambda}\left[\lambda \bar{h}^{(9)}+\bar{h}^{(10)}\right] \\
& f_{7}^{r}=\frac{u^{r} \hat{\mathcal{L}}^{3}}{2 l(l+1) \lambda} \bar{h}^{(10)}
\end{aligned}
$$

\section{Appendix D: Useful identities}

The following identities are used in deriving Eq. (2.25) for the full-force modes. In these relations $Y^{l m}$ are the standard spherical harmonics, and the identities are valid for any values of $l, m$.

$$
\begin{aligned}
\sin ^{2} \theta Y^{l m} & =\alpha_{(+2)}^{l m} Y^{l+2, m}+\alpha_{(0)}^{l m} Y^{l m}+\alpha_{(-2)}^{l m} Y^{l-2, m}, \\
\cos \theta \sin \theta Y_{, \theta}^{l m} & =\beta_{(+2)}^{l m} Y^{l+2, m}+\beta_{(0)}^{l m} Y^{l m}+\beta_{(-2)}^{l m} Y^{l-2, m} \\
\sin ^{2} \theta Y_{, \theta \theta}^{l m} & =\gamma_{(+2)}^{l m} Y^{l+2, m}+\gamma_{(0)}^{l m} Y^{l m}+\gamma_{(-2)}^{l m} Y^{l-2, m}, \\
\sin \theta Y_{, \theta}^{l m} & =\delta_{(+1)}^{l m} Y^{l+1, m}+\delta_{(-1)}^{l m} Y^{l-1, m}, \\
\cos \theta Y^{l m}-\sin \theta Y_{, \theta}^{l m} & =\epsilon_{(+1)}^{l m} Y^{l+1, m}+\epsilon_{(-1)}^{l m} Y^{l-1, m} \\
\sin ^{3} \theta Y_{, \theta}^{l m} & =\zeta_{(+3)}^{l m} Y^{l+3, m}+\zeta_{(+1)}^{l m} Y^{l+1, m}+\zeta_{(-1)}^{l m} Y^{l-1, m}+\zeta_{(-3)}^{l m} Y^{l-3, m}, \\
\cos \theta \sin ^{2} \theta Y_{, \theta \theta}^{l m} & =\xi_{(+3)}^{l m} Y^{l+3, m}+\xi_{+}^{l m} Y^{l+1, m}+\xi_{(-1)}^{l m} Y^{l-1, m}+\xi_{(-3)}^{l m} Y^{l-3, m}
\end{aligned}
$$


Here the various coefficients are all constructed from

$$
C_{l m}=\left[\frac{l^{2}-m^{2}}{(2 l+1)(2 l-1)}\right]^{1 / 2}
$$

using

$$
\begin{gathered}
\alpha_{(+2)}^{l m}=-C_{l+1, m} C_{l+2, m}, \quad \alpha_{(0)}^{l m}=1-C_{l m}^{2}-C_{l+1, m}^{2}, \quad \alpha_{(-2)}^{l m}=-C_{l m} C_{l-1, m}, \\
\beta_{(+2)}^{l m}=l C_{l+1, m} C_{l+2, m}, \quad \beta_{(0)}^{l m}=l C_{l+1, m}^{2}-(l+1) C_{l m}^{2}, \quad \beta_{(-2)}^{l m}=-(l+1) C_{l m} C_{l-1, m}, \\
\gamma_{(+2)}^{l m}=l^{2} C_{l+1, m} C_{l+2, m}, \quad \gamma_{(0)}^{l m}=m^{2}-l(l+1)+l^{2} C_{l+1, m}^{2}+(l+1)^{2} C_{l m}^{2}, \quad \gamma_{(-2)}^{l m}=(l+1)^{2} C_{l m} C_{l-1, m}, \\
{ }_{(+1)}^{l m}=l C_{l+1, m}, \quad \delta_{(-1)}^{l m}=-(l+1) C_{l m}, \\
\epsilon_{(+1)}^{l m}=(1-l) C_{l+1, m}, \quad \epsilon_{(-1)}^{l m}=(l+2) C_{l m}, \\
\zeta_{(+3)}^{l m}=-l C_{l+1, m} C_{l+2, m} C_{l+3, m}, \\
\zeta_{(+1)}^{l m}=C_{l+1, m}\left[l\left(1-C_{l+1, m}^{2}-C_{l+2, m}^{2}\right)+(l+1) C_{l, m}^{2}\right], \\
\zeta_{(-1)}^{l m}=-C_{l, m}\left[(l+1)\left(1-C_{l-1, m}^{2}-C_{l, m}^{2}\right)+l C_{l+1, m}^{2}\right], \\
\zeta_{(-3)}^{l m}=(l+1) C_{l, m} C_{l-1, m} C_{l-2, m}, \\
\xi_{(+3)}^{l m}=l^{2} C_{l+1, m} C_{l+2, m} C_{l+3, m}, \\
\xi_{(+1)}^{l m}=C_{l+1, m}\left[m^{2}-l(l+1)+l^{2} C_{l+1, m}^{2}+(l+1)^{2} C_{l, m}^{2}+l^{2} C_{l+2, m}^{2}\right], \\
\xi_{(-1)}^{l m}=C_{l, m}\left[m^{2}-l(l+1)+l^{2} C_{l+1, m}^{2}+(l+1)^{2} C_{l, m}^{2}+(l+1)^{2} C_{l-1, m}^{2}\right] \\
\xi_{(-3)}^{l m}=(l+1)^{2} C_{l, m} C_{l-1, m} C_{l-2, m} .
\end{gathered}
$$

\section{Appendix E: Jump conditions for the perturbation modes and their derivatives}

As explained in Sec. IIIB, our finite-difference scheme makes use of formal jump conditions for the perturbation modes $\bar{h}^{(i) l m}$ and their (first through fourth) derivatives across the particle's orbit. In this appendix we derive the necessary conditions. Our derivation refers to a specific (yet generic) point $x_{0}$ along the orbit, with known coordinates $\left(r_{0}, t_{0}\right)$ or $\left(u_{0}, v_{0}\right)$, and velocity components $\dot{u}_{0} \equiv d u_{\mathrm{p}} /\left.d \tau\right|_{x_{0}}$ and $\dot{v}_{0} \equiv d v_{\mathrm{p}} /\left.d \tau\right|_{x_{0}}$, where $\tau$ is proper time along the orbit. We will use the notation $[A]_{0} \equiv A\left(x_{0}^{+}\right)-A\left(x_{0}^{-}\right)$, where $A\left(x_{0}^{ \pm}\right)$are the values of a $1+1 \mathrm{D}$ field $A(r, t)$ calculated by taking the limits $t \rightarrow t_{0}$ and $r \rightarrow r_{0}^{ \pm}$. In this appendix we shall omit multipolar indices $l m$ for brevity.

\section{Continuity condition for $\bar{h}^{(i)}$}

The Lorenz-gauge perturbation modes $\bar{h}^{(i)}$ are all continuous at any point along the orbit. This can be verified, for example, by noticing that the distributional form $\bar{h}^{(i)}=\bar{h}_{+}^{(i)}(r, t) \theta\left[r-r_{\mathrm{p}}(t)\right]+\bar{h}_{-}^{(i)}(r, t) \theta\left[r_{\mathrm{p}}(t)-r\right]$ is indeed a solution of the perturbation equations (2.20) only if the homogeneous solutions $\bar{h}_{+}^{(i)}$ and $\bar{h}_{-}^{(i)}$ satisfy $\bar{h}_{+}^{(i)}=\bar{h}_{-}^{(i)}$ along the worldline. Hence, for any $i$ we have

$$
\left[\bar{h}^{(i)}\right]_{0}=0
$$




\section{Jump conditions for the 1st derivatives}

Let us we re-express the field equations (2.20) in the form

$$
\bar{h}_{, u v}^{(i)}+P^{(i)}=\int d \tau \tilde{S}^{(i)}\left(x_{\mathrm{p}}(\tau)\right) \delta\left(u-u_{\mathrm{p}}(\tau)\right) \delta\left(v-v_{\mathrm{p}}(\tau)\right)
$$

where

$$
P^{(i)} \equiv V(r) \bar{h}^{(i)}+\mathcal{M}_{(j)}^{(i)} \bar{h}^{(j)}, \quad \tilde{S}^{(i)} \equiv 2 \mathcal{E} f_{\mathrm{p}}^{-2} S^{(i)},
$$

with $\mathcal{M}_{(j)}^{(i)}$ and $S^{(i)}$ being the quantities given in Appendix B, and $V(r)$ being defined in Eq. (2.21). Now consider formally integrating Eq. (E2) along the ray $v=v_{0}$ over an infinitesimal interval $\left(u_{0}-\epsilon, u_{0}+\epsilon\right)$ across $x_{0}$. The integral of the left-hand side yields

$$
\left.\bar{h}_{, v}^{(i)}\right|_{u_{0}-\epsilon} ^{u_{0}+\epsilon}+\int_{u_{0}-\epsilon}^{u_{0}+\epsilon} P^{(i)} d u \quad \rightarrow \quad-\left[\bar{h}_{, v}^{(i)}\right]_{0}
$$

as $\epsilon \rightarrow 0$, since $P^{(i)}$ is bounded. Similarly integrating the right-hand side of Eq. (E2) one finds

$$
\int_{u_{0}-\epsilon}^{u_{0}+\epsilon} d u \int d \tau \tilde{S}^{(i)}\left(x_{\mathrm{p}}(\tau)\right) \delta\left[u-u_{\mathrm{p}}(\tau)\right] \delta\left[v_{0}-v_{\mathrm{p}}(\tau)\right]=\dot{v}_{0}^{-1} \tilde{S}^{(i)}\left(x_{0}\right) .
$$

The desired jump condition is therefore

$$
\left[\bar{h}_{, v}^{(i)}\right]_{0}=-\dot{v}_{0}^{-1} \tilde{S}^{(i)}\left(x_{0}\right) .
$$

Similarly integrating the field equations along $u=u_{0}$ over an infinitesimal interval $\left(v_{0}-\epsilon, v_{0}+\epsilon\right)$ one also obtains

$$
\left[\bar{h}_{, u}^{(i)}\right]_{0}=\dot{u}_{0}^{-1} \tilde{S}^{(i)}\left(x_{0}\right) .
$$

\section{Jump conditions for the 2nd derivatives}

It is straightforward to derive the jump condition in the mixed uv-derivative. From Eq. (E2) we immediately have

$$
\left[\partial_{u} \partial_{v} \bar{h}^{(i)}\right]_{0}=-\left[P^{(i)}\right]_{0}
$$

as the source term is supported only on the worldline. Recall $P^{(i)}$ involves the perturbation $\bar{h}^{(i)}$ and its first derivatives only, and so the jump in $P^{(i)}$ can be readily calculated from the jump conditions obtained above.

To derive a jump condition for the $v v$-derivative, we first take the $v$ derivatives of the field equations (E2), and then integrate with respect to $u$ along $v=v_{0}$ over $\left(u_{0}-\epsilon, u_{0}+\epsilon\right)$. We thereby obtain

$$
-\left[\bar{h}_{, v v}^{(i)}\right]_{0}=\lim _{\epsilon \rightarrow 0} \int_{u_{0}-\epsilon}^{u_{0}+\epsilon} d u\left[-P_{, v}^{(i)}\left(u, v_{0}\right)+\int d \tau \tilde{S}^{(i)}\left(x_{\mathrm{p}}\right) \delta^{\prime}\left[v_{0}-v_{\mathrm{p}}(\tau)\right] \delta\left[u-u_{\mathrm{p}}(\tau)\right]\right] .
$$

Now, for $P^{(i)}$ we may write

$$
P^{(i)}=P_{+}^{(i)}(u, v) \theta\left[v-v_{\mathrm{p}}(u)\right]+P_{-}^{(i)}(u, v) \theta\left[v_{\mathrm{p}}(u)-v\right],
$$

in which $P_{ \pm}^{(i)}$ are smooth and where $v_{\mathrm{p}}(u)$ is the value of $v$ at which the outgoing ray of retarded time $u$ intersects the worldline. Using this form we obtain

$$
\begin{aligned}
\int_{u_{0}-\epsilon}^{u_{0}+\epsilon} P_{, v}^{(i)}\left(u, v_{0}\right) d u= & \int_{u_{0}-\epsilon}^{u_{0}+\epsilon} d u\left\{\left(P_{+}^{(i)}\left(u, v_{0}\right)-P_{-}^{(i)}\left(u, v_{0}\right)\right) \delta\left[v_{0}-v_{\mathrm{p}}(u)\right]\right. \\
& \left.+P_{+, v}^{(i)}\left(u, v_{0}\right) \theta\left[v_{0}-v_{\mathrm{p}}(u)\right]+P_{-, v}^{(i)}\left(u, v_{0}\right) \theta\left[v_{\mathrm{p}}(u)-v_{0}\right]\right\} \rightarrow \quad\left(\dot{u}_{0} / \dot{v}_{0}\right)\left[P^{(i)}\right]_{0}
\end{aligned}
$$


as $\epsilon \rightarrow 0$, since $P_{ \pm, v}^{(i)}$ are bounded. Lastly, for the integral involving $\tilde{S}^{(i)}$ in Eq. (E9) we have

$$
\begin{aligned}
\int_{u_{0}-\epsilon}^{u_{0}+\epsilon} d u & \int d \tau \tilde{S}^{(i)}\left(x_{\mathrm{p}}(\tau)\right) \delta^{\prime}\left[v_{0}-v_{\mathrm{p}}(\tau)\right] \delta\left[u-u_{\mathrm{p}}(\tau)\right] \\
= & \int d \tau \tilde{S}^{(i)}\left(x_{\mathrm{p}}(\tau)\right) \delta^{\prime}\left[v_{0}-v_{\mathrm{p}}(\tau)\right] \theta\left[u_{\mathrm{p}}(\tau)-\left(u_{0}-\epsilon\right)\right] \theta\left[\left(u_{0}+\epsilon\right)-u_{\mathrm{p}}(\tau)\right] \\
= & \int_{\tau_{0}-\delta_{-}}^{\tau_{0}+\delta_{+}} d \tau \tilde{S}^{(i)}\left(x_{\mathrm{p}}(\tau)\right) \delta^{\prime}\left[v_{0}-v_{\mathrm{p}}(\tau)\right] \\
= & -\int_{\tau_{0}-\delta_{-}}^{\tau_{0}+\delta_{+}} d \tau \tilde{S}^{(i)}\left(x_{\mathrm{p}}\right) \dot{v}_{\mathrm{p}}^{-1} \frac{d}{d \tau} \delta\left[v_{\mathrm{p}}(\tau)-v_{0}\right] \\
= & \int_{\tau_{0}-\delta_{-}}^{\tau_{0}+\delta_{+}} d \tau \frac{d}{d \tau}\left[\dot{v}_{\mathrm{p}}^{-1} \tilde{S}^{(i)}\left(x_{\mathrm{p}}(\tau)\right)\right] \delta\left[v_{\mathrm{p}}(\tau)-v_{0}\right] \\
= & \left.\dot{v}_{0}^{-1} \frac{d}{d \tau}\left[\dot{v}_{\mathrm{p}}^{-1} \tilde{S}^{(i)}\left(x_{\mathrm{p}}\right)\right]\right|_{x_{0}}
\end{aligned}
$$

where $\tau_{0}$ is the value of $\tau$ at $x_{0}$, and $\delta_{ \pm}$are the values of $\tau$ at which the two outgoing rays of constant retarded times $u_{0} \pm \epsilon$ cross the worldline. Combining the above results, we arrive at

$$
\left[\bar{h}_{, v v}^{(i)}\right]_{0}=\left(\dot{u}_{0} / \dot{v}_{0}\right)\left[P^{(i)}\right]_{0}-\left.\dot{v}_{0}^{-1} \frac{d}{d \tau}\left[\dot{v}_{\mathrm{p}}^{-1} \tilde{S}^{(i)}\left(x_{\mathrm{p}}\right)\right]\right|_{x_{0}} .
$$

The jump condition for the $u u$-derivatives is derived in a similar fashion. The result is

$$
\left[\bar{h}_{, u u}^{(i)}\right]_{0}=\left(\dot{v}_{0} / \dot{u}_{0}\right)\left[P^{(i)}\right]_{0}+\left.\dot{u}_{0}^{-1} \frac{d}{d \tau}\left[\dot{u}_{\mathrm{p}}^{-1} \tilde{S}^{(i)}\left(x_{\mathrm{p}}\right)\right]\right|_{x_{0}} .
$$

\section{Jump conditions for the 3rd derivatives}

The jumps in the two mixed third derivatives are readily obtained by considering the derivatives of the field equations (E2) with respect to $v$ and with respect to $u$ :

$$
\begin{aligned}
& {\left[\bar{h}_{, v v u}^{(i)}\right]_{0}=-\left[P_{, v}^{(i)}\right]_{0},} \\
& {\left[\bar{h}_{, u u v}^{(i)}\right]_{0}=-\left[P_{, u}^{(i)}\right]_{0} .}
\end{aligned}
$$

Here the jumps in $P_{, u}^{(i)}$ and $P_{, v}^{(i)}$ can be obtained from the jumps in the first and second derivatives of $\bar{h}^{(i)}$ derived above.

To obtain the jump condition for the $v v v$ derivative, we differentiate the field equations (E2) twice with respect to $v$, and then integrate with respect to $u$ along $v=v_{0}$ over $\left(u_{0}-\epsilon, u_{0}+\epsilon\right)$. This yields

$$
-\left[\bar{h}_{, v v v}^{(i)}\right]_{0}=\lim _{\epsilon \rightarrow 0} \int_{u_{0}-\epsilon}^{u_{0}+\epsilon} d u\left[-P_{, v v}^{(i)}\left(u, v_{0}\right)+\int d \tau \tilde{S}^{(i)}\left(x_{\mathrm{p}}\right) \delta^{\prime \prime}\left[v_{0}-v_{\mathrm{p}}(\tau)\right] \delta\left[u-u_{\mathrm{p}}(\tau)\right]\right] .
$$

Using Eq. (E10) again, we obtain

$$
\begin{aligned}
\int_{u_{0}-\epsilon}^{u_{0}+\epsilon} P_{, v v}^{(i)}\left(u, v_{0}\right) d u= & \int_{u_{0}-\epsilon}^{u_{0}+\epsilon} d u\left\{2\left(P_{+, v}^{(i)}\left(u, v_{0}\right)-P_{-, v}^{(i)}\left(u, v_{0}\right)\right) \delta\left[v_{0}-v_{\mathrm{p}}(u)\right]\right. \\
& +\left(P_{+}^{(i)}\left(u, v_{0}\right)-P_{-}^{(i)}\left(u, v_{0}\right)\right) \delta^{\prime}\left[v_{0}-v_{\mathrm{p}}(u)\right] \\
& \left.+P_{+, v v}^{(i)}\left(u, v_{0}\right) \theta\left[v_{0}-v_{\mathrm{p}}(u)\right]+P_{-, v v}^{(i)}\left(u, v_{0}\right) \theta\left[v_{\mathrm{p}}(u)-v_{0}\right]\right\} \\
& \rightarrow 2\left(v_{0}^{\prime}\right)^{-1}\left[P_{, v}^{(i)}\right]_{0}+\left(v_{0}^{\prime}\right)^{-2}\left[P_{, u}^{(i)}\right]_{0}+u_{0}^{\prime \prime}\left[P^{(i)}\right]_{0}
\end{aligned}
$$


as $\epsilon \rightarrow 0$. Here we have introduced $v_{\mathrm{p}}^{\prime} \equiv d v_{\mathrm{p}}(u) / d u$ and $v_{0}^{\prime} \equiv v_{\mathrm{p}}^{\prime}\left(u_{0}\right)=\dot{v}_{0} / \dot{u}_{0}$; and similarly, defining $u_{\mathrm{p}}(v)$ as the value of $u$ at which the incoming ray with advanced time $v$ intersects the worldline, we introduced $u_{\mathrm{p}}^{\prime} \equiv d u_{\mathrm{p}}(v) / d v$ and $u_{\mathrm{p}}^{\prime \prime} \equiv d^{2} u_{\mathrm{p}}(v) / d v^{2}$, with $u_{0}^{\prime} \equiv u_{\mathrm{p}}^{\prime}\left(v_{0}\right)=\dot{u}_{0} / \dot{v}_{0}$ and $u_{0}^{\prime \prime} \equiv u_{\mathrm{p}}^{\prime \prime}\left(v_{0}\right)=\left(\ddot{u}_{0} \dot{v}_{0}-\dot{u}_{0} \ddot{v}_{0}\right) / \dot{v}_{0}^{3}$. To evaluate the limit in Eq. (E18) we have used

$$
\int_{u_{0}-\epsilon}^{u_{0}+\epsilon} d u\left(P_{+, v}^{(i)}\left(u, v_{0}\right)-P_{-, v}^{(i)}\left(u, v_{0}\right)\right) \delta\left[v_{0}-v_{\mathrm{p}}(u)\right] \rightarrow\left(v_{0}^{\prime}\right)^{-1}\left[P_{, v}^{(i)}\right]_{0}
$$

$($ as $\epsilon \rightarrow 0)$, along with

$$
\begin{aligned}
\int_{u_{0}-\epsilon}^{u_{0}+\epsilon} d u & \left(P_{+}^{(i)}\left(u, v_{0}\right)-P_{-}^{(i)}\left(u, v_{0}\right)\right) \delta^{\prime}\left[v_{0}-v_{\mathrm{p}}(u)\right] \\
= & -\int_{u_{0}-\epsilon}^{u_{0}+\epsilon} d u\left\{\left(P_{+}^{(i)}\left(u, v_{0}\right)-P_{-}^{(i)}\left(u, v_{0}\right)\right)\left(v_{\mathrm{p}}^{\prime}\right)^{-1} \frac{d}{d u}\left[\left(v_{\mathrm{p}}^{\prime}\right)^{-1} \delta\left(u-u_{\mathrm{p}}(v)\right)\right]\right\} \\
& =\int_{u_{0}-\epsilon}^{u_{0}+\epsilon} d u\left\{\frac{d}{d u}\left[\left(P_{+}^{(i)}\left(u, v_{0}\right)-P_{-}^{(i)}\left(u, v_{0}\right)\right)\left(v_{\mathrm{p}}^{\prime}\right)^{-1}\right]\left(v_{\mathrm{p}}^{\prime}\right)^{-1} \delta\left(u-u_{\mathrm{p}}(v)\right)\right\} \\
& \rightarrow\left(v_{0}^{\prime}\right)^{-2}\left[P_{, u}^{(i)}\right]_{0}-v_{0}^{\prime \prime}\left(v_{0}^{\prime}\right)^{-3}\left[P^{(i)}\right]_{0} \\
& =\left(v_{0}^{\prime}\right)^{-2}\left[P_{, u}^{(i)}\right]_{0}+u_{0}^{\prime \prime}\left[P^{(i)}\right]_{0} .
\end{aligned}
$$

The term involving $\tilde{S}^{(i)}$ in Eq. (E17) gives, upon twice integrating by parts,

$$
\left.\int_{u_{0}-\epsilon}^{u_{0}+\epsilon} d u \int d \tau \tilde{S}^{(i)}\left(x_{\mathrm{p}}\right) \delta^{\prime \prime}\left(v_{0}-v_{\mathrm{p}}(\tau)\right) \delta\left(u-u_{\mathrm{p}}(\tau)\right) \rightarrow \dot{v}_{0}^{-1} \frac{d}{d \tau}\left[\dot{v}_{\mathrm{p}}^{-1} \frac{d}{d \tau}\left(\dot{v}_{\mathrm{p}}^{-1} \tilde{S}^{(i)}\right)\right]\right|_{x_{0}}
$$

as $\epsilon \rightarrow 0$. Finally, substituting from Eqs. (E18) and (E21) in Eq. (E17) we arrive at

$$
\left[\bar{h}_{, v v v}^{(i)}\right]_{0}=2\left(v_{0}^{\prime}\right)^{-1}\left[P_{, v}^{(i)}\right]_{0}+\left(v_{0}^{\prime}\right)^{-2}\left[P_{, u}^{(i)}\right]_{0}+u_{0}^{\prime \prime}\left[P^{(i)}\right]_{0}-\left.\dot{v}_{0}^{-1} \frac{d}{d \tau}\left[\dot{v}_{\mathrm{p}}^{-1} \frac{d}{d \tau}\left(\dot{v}_{\mathrm{p}}^{-1} \tilde{S}^{(i)}\right)\right]\right|_{x_{0}} .
$$

The jumps $\left[P_{, u}^{(i)}\right]_{0}$ and $\left[P_{, v}^{(i)}\right]_{0}$ can be obtained in a straightforward way from the jumps in the first and second derivatives of the perturbation, obtained in previous steps.

In a precisely analogous manner, differentiating the field equations (E2) twice with respect to $u$ and then integrating with respect to $v$ along $u=u_{0}$ over $\left(v_{0}-\epsilon, v_{0}+\epsilon\right)$ gives

$$
\left[\bar{h}_{, \text {uuu }}^{(i)}\right]_{0}=2\left(u_{0}^{\prime}\right)^{-1}\left[P_{, u}^{(i)}\right]_{0}+\left(u_{0}^{\prime}\right)^{-2}\left[P_{, v}^{(i)}\right]_{0}+v_{0}^{\prime \prime}\left[P^{(i)}\right]_{0}+\left.\dot{u}_{0}^{-1} \frac{d}{d \tau}\left[\dot{u}_{\mathrm{p}}^{-1} \frac{d}{d \tau}\left(\dot{u}_{\mathrm{p}}^{-1} \tilde{S}^{(i)}\right)\right]\right|_{x_{0}},
$$

where $v_{0}^{\prime \prime} \equiv v_{\mathrm{p}}^{\prime \prime}\left(u_{0}\right)=\left(\ddot{v}_{0} \dot{u}_{0}-\dot{v}_{0} \ddot{u}_{0}\right) / \dot{u}_{0}^{3}$.

\section{Jump conditions for the 4 th derivatives}

The jumps in the five 4th-order partial derivatives of $\bar{h}^{(i)}$ are obtained in a similar manner. The results are

$$
\begin{gathered}
{\left[\bar{h}_{, u v v v}^{(i)}\right]_{0}=-\left[P_{, v v}^{(i)}\right]_{0}, \quad\left[\bar{h}_{, u u v v}^{(i)}\right]_{0}=-\left[P_{, u v}^{(i)}\right]_{0}, \quad\left[\bar{h}_{, u u u v}^{(i)}\right]_{0}=-\left[P_{, u u}^{(i)}\right]_{0},} \\
{\left[\bar{h}_{, v v v v}^{(i)}\right]_{0}=u_{0}^{\prime \prime \prime}\left[P^{(i)}\right]_{0}+3 u_{0}^{\prime \prime}\left[P_{, v}^{(i)}\right]_{0}+3 u_{0}^{\prime \prime}\left(v_{0}^{\prime}\right)^{-1}\left[P_{, u}^{(i)}\right]_{0}+3\left(v_{0}^{\prime}\right)^{-1}\left[P_{, v v}^{(i)}\right]_{0}+3\left(v_{0}^{\prime}\right)^{-2}\left[P_{, u v}^{(i)}\right]_{0}} \\
+\left(v_{0}^{\prime}\right)^{-3}\left[P_{, u u}^{(i)}\right]_{0}-\left.\dot{v}_{0}^{-1} \frac{d}{d \tau}\left\{\dot{v}_{\mathrm{p}}^{-1} \frac{d}{d \tau}\left[\dot{v}_{\mathrm{p}}^{-1} \frac{d}{d \tau}\left(\dot{v}_{\mathrm{p}}^{-1} \tilde{S}^{(i)}\right)\right]\right\}\right|_{x_{0}} \\
{\left[\bar{h}_{, u u u u}^{(i)}\right]_{0}=} \\
\quad v_{0}^{\prime \prime \prime}\left[P^{(i)}\right]_{0}+3 v_{0}^{\prime \prime}\left[P_{, u}^{(i)}\right]_{0}+3 v_{0}^{\prime \prime}\left(u_{0}^{\prime}\right)^{-1}\left[P_{, v}^{(i)}\right]_{0}+3\left(u_{0}^{\prime}\right)^{-1}\left[P_{, u u}^{(i)}\right]_{0}+3\left(u_{0}^{\prime}\right)^{-2}\left[P_{, u v}^{(i)}\right]_{0} \\
+\left(u_{0}^{\prime}\right)^{-3}\left[P_{, v v}^{(i)}\right]_{0}+\left.\dot{u}_{0}^{-1} \frac{d}{d \tau}\left\{\dot{u}_{\mathrm{p}}^{-1} \frac{d}{d \tau}\left[\dot{u}_{\mathrm{p}}^{-1} \frac{d}{d \tau}\left(\dot{u}_{\mathrm{p}}^{-1} \tilde{S}^{(i)}\right)\right]\right\}\right|_{x_{0}}
\end{gathered}
$$


Here $u_{0}^{\prime \prime \prime}=u_{\mathrm{p}}^{\prime \prime \prime}\left(r_{0}\right)=\left(\dddot{u}_{0} \dot{v}_{0}^{2}-3 \ddot{u}_{0} \ddot{v}_{0} \dot{v}_{0}+3 \dot{u}_{0} \ddot{v}_{0}^{2}-\dot{u}_{0} \dddot{v}_{0} \dot{v}_{0}\right) / \dot{v}_{0}^{5}$ and $v_{0}^{\prime \prime \prime}=v_{\mathrm{p}}^{\prime \prime \prime}\left(r_{0}\right)=\left(\dddot{v}_{0} \dot{u}_{0}^{2}-3 \ddot{v}_{0} \ddot{u}_{0} \dot{u}_{0}+3 \dot{v}_{0} \ddot{u}_{0}^{2}-\dot{v}_{0} \dddot{u}_{0} \dot{u}_{0}\right) / \dot{u}_{0}^{5}$. The jumps in the various $P^{(i)}$ terms are related to the jumps in the 1 st, 2 nd, and 3rd derivatives of the perturbation, which were obtained in previous steps.

\section{Appendix F: Source terms and jump conditions for slightly eccentric orbits}

\section{Source terms}

We give here explicit expressions for the $O(e)$ source coefficients $S_{1}^{(i)}$ defined in Eq. (5.27). These are needed in our discussion of the $e$-expansion method in Sec. $\nabla$. We use the notation

$$
\mathcal{E}_{0} \equiv \mathcal{E}\left(p=r_{0}, e=0\right)=\frac{r_{0}-2 M}{\sqrt{r_{0}\left(r_{0}-3 M\right)}}, \quad \mathcal{L}_{0} \equiv \mathcal{L}\left(p=r_{0}, e=0\right)=\frac{\sqrt{M} r_{0}}{\sqrt{r_{0}-3 M}}
$$

along with $f_{0} \equiv 1-2 M / r_{0}$, and omit multipolar indices $l, m$ for brevity.

The coefficients $S_{1}^{(i)}$ are obtained by formally expanding the source terms $S^{(i)}$ given in Eqs. (B11)-(B20) in powers of $e$ through $O(e)$, assuming the e-expansion forms of the trajectory,

$$
r_{\mathrm{p}}(\tau)=r_{0}\left(1-e \cos \omega_{r} \tau\right)+O\left(e^{2}\right), \quad \varphi_{\mathrm{p}}(\tau)=\omega_{\varphi} \tau+\frac{2 \omega_{\varphi}}{\omega_{r}} e \sin \omega_{r} \tau+O\left(e^{2}\right),
$$

where we wrote $\omega_{\varphi}=\mathcal{L}_{0} / r_{0}^{2} . S_{1}^{(i)}$ in then obtained as the linear variation of $S^{(i)}$ with respect to $e$ [recall Eq. (5.27)]. The result can be expressed in the form

$$
S_{1}^{(i)}=\sum_{n= \pm 1} \mathcal{S}_{1, n}^{(i)} e^{-i \omega_{n m} \tau}
$$

where $\omega_{n m}=n \omega_{r}+m \omega_{\varphi}$ and the various coefficients are

$$
\begin{aligned}
\mathcal{S}_{1, n}^{(1)} & =\frac{2 \pi\left(r_{0}-2 M\right)^{3}}{\mathcal{E}_{0} r_{0}^{4}\left(r_{0}-3 M\right)}\left[r_{0}-6 M+2 m n\left(r_{0}-2 M\right) \frac{\omega_{\varphi}}{\omega_{r}}\right] Y_{l m}^{*}(0), \\
\mathcal{S}_{1, n}^{(2)} & =-4 \pi i n \omega_{r} f_{0}^{2} Y_{l m}^{*}(0) \\
\mathcal{S}_{1, n}^{(3)} & =\frac{2 \pi \mathcal{E}_{0}}{r_{0}^{2}}\left[r_{0}-4 M+2 m n\left(r_{0}-2 M\right) \frac{\omega_{\varphi}}{\omega_{r}}\right] Y_{l m}^{*}(0), \\
\mathcal{S}_{1, n}^{(4)}= & \frac{8 \pi i m \mathcal{L}_{0}\left(r_{0}-2 M\right)}{r_{0}^{4}}\left[r_{0}-4 M+m n\left(r_{0}-2 M\right) \frac{\omega_{\varphi}}{\omega_{r}}\right] Y_{l m}^{*}(0), \\
\mathcal{S}_{1, n}^{(5)}= & \frac{4 \pi m n \omega_{r} \mathcal{L}_{0}\left(r_{0}-2 M\right)^{2}}{\mathcal{E}_{0} r_{0}^{3}} Y_{l m}^{*}(0), \\
\mathcal{S}_{1, n}^{(6)}= & \frac{2 \pi \mathcal{L}_{0}^{2}\left(r_{0}-2 M\right)}{\mathcal{E}_{0} r_{0}^{5}}\left[3 r_{0}-10 M+2 m n\left(r_{0}-2 M\right) \frac{\omega_{\varphi}}{\omega_{r}}\right] Y_{l m}^{*}(0), \\
\mathcal{S}_{1, n}^{(7)}= & {\left[l(l+1)-2 m^{2}\right] \mathcal{S}_{1, n}^{(6)}, } \\
\mathcal{S}_{1, n}^{(8)}= & \frac{8 \pi \mathcal{L}_{0}\left(r_{0}-2 M\right)}{r_{0}^{4}}\left[r_{0}-4 M+m n\left(r_{0}-2 M\right) \frac{\omega_{\varphi}}{\omega_{r}}\right] Y_{l m, \theta}^{*}(0), \\
\mathcal{S}_{1, n}^{(9)}= & -\frac{4 \pi i n \omega_{r} \mathcal{L}_{0}\left(r_{0}-2 M\right)^{2}}{\mathcal{E}_{0} r_{0}^{3}} Y_{l m, \theta}^{*}(0), \\
\mathcal{S}_{1, n}^{(10)}= & \frac{4 \pi i m \mathcal{L}_{0}^{2}\left(r_{0}-2 M\right)^{2}}{\mathcal{E}_{0} r_{0}^{5}}\left[3 r_{0}-10 M+2 m n\left(r_{0}-2 M\right) \frac{\omega_{\varphi}}{\omega_{r}}\right] Y_{l m, \theta}^{*}(0),
\end{aligned}
$$

Here $\omega_{n m}=n \omega_{r}+m \omega_{\varphi}$, with $Y_{l m}^{*}(0)$ and $Y_{l m, \theta}^{*}(0)$ denoting the spherical harmonics and their $\theta$-derivatives evaluated at $\theta=\pi / 2$ and $\varphi=0$.

Notice that if we also write

$$
T_{1}^{(i)}=\sum_{n= \pm 1} \mathcal{T}_{1, n}^{(i)} e^{-i \omega_{n m} \tau} \quad \text { with } \quad \mathcal{T}_{1, n}^{(i)}=\frac{1}{2} r_{0} S_{0}^{(i)}\left(\varphi_{\mathrm{p}}=0\right)
$$


then we may split the $O(e)$ part of the metric perturbation into two harmonic components, $\bar{h}_{1}^{(i)}=\bar{h}_{1,+1}^{(i)}+\bar{h}_{1,-1}^{(i)}$, each of which satisfying the field equation

$$
\square \bar{h}_{1, n}^{(i)}+\mathcal{M}_{(j)}^{(i) l} \bar{h}_{1, n}^{(j)}=\left[\mathcal{S}_{1, n}^{(i)} \delta\left(r-r_{0}\right)+\mathcal{T}_{1, n}^{(i)} \delta^{\prime}\left(r-r_{0}\right)\right] e^{-i \omega_{n m} \tau} \quad(n= \pm 1) .
$$

Evidently, the source for $\bar{h}_{1, n}^{(i)}$ is simple harmonic in $\tau$, with frequency $\omega_{n m}$. It is clear that the solutions $\bar{h}_{1, n}^{(i)}(r, t)$ (and their derivatives) will inherit this simple harmonic dependence on $\tau$ along the orbit. Hence we may write, for example

$$
\left.\partial_{t} \bar{h}_{1, n}^{(i)}\right|_{r_{\mathrm{p}}(\tau)}=-\left.i \omega_{n m} f_{0} \mathcal{E}_{0}^{-1} \bar{h}_{1, n}^{(i)}\right|_{r_{\mathrm{p}}(\tau)},
$$

where the factor $f_{0} \mathcal{E}_{0}^{-1}$ is simply $\left(d t_{\mathrm{p}} / d \tau\right)^{-1}$.

\section{Jump conditions}

Here we explain the derivation of the necessary jump conditions for the variables $\bar{h}_{1}^{(i)}$ associated with the $O(e)$ piece of the metric perturbation [recall Eq. (5.28)]. Unlike the full physical metric perturbation functions $\bar{h}^{(i)}$ [or the $O\left(e^{0}\right)$ variables $\left.\bar{h}_{0}^{(i)}\right]$, the functions $\bar{h}_{1}^{(i)}$ are in general discontinuous across the particle's worldline, because the field equation which defines them, Eq. (5.30), is sourced by a derivative of a delta function. For our TD algorithm we need to derive analytic jump conditions for $\bar{h}_{1}^{(i)}$ itself as well as for its first to fourth derivatives along the orbit. Our task here will be somewhat simpler than in the case of the full perturbation $\bar{h}^{(i)}$ (analyzed in Appendix E) thanks to the simple harmonic dependence of $\bar{h}_{1}^{(i)}$ on $t$ along the orbit, expressed in Eq. (F15).

We start by reexpressing the field equations (ㅍ14) in the form

$$
\left(\partial_{r_{*}}^{2}-\partial_{t}^{2}\right) \bar{h}_{1, n}^{(i)}-4 P_{1, n}^{(i)}=-4\left[\mathcal{S}_{1, n}^{(i)} \delta\left(r-r_{0}\right)+\mathcal{T}_{1, n}^{(i)} \delta^{\prime}\left(r-r_{0}\right)\right] e^{-i \omega_{n m} \tau} \quad(n= \pm 1),
$$

where the definition of $P_{1, n}^{(i)}$ is similar to that of $P^{(i)}$ in Eq. (E3), simply replacing $\bar{h}^{(i)} \rightarrow \bar{h}_{1, n}^{(i)}$. Substituting

$$
\bar{h}_{1, n}^{(i)}=\bar{h}_{1, n}^{(i)-} \theta\left(r_{0}-r\right)+\bar{h}_{1, n}^{(i)+} \theta\left(r-r_{0}\right)
$$

and comparing the $\delta^{\prime}\left(r-r_{0}\right)$ and $\delta\left(r-r_{0}\right)$-terms on both sides of the resulting equation, we readily obtain the jump formulas

$$
\begin{aligned}
{\left[\bar{h}_{1, n}^{(i)}\right]_{0} } & =-\frac{4}{f_{0}^{2}} \mathcal{T}_{1, n} e^{-i \omega_{n m} \tau}, \\
{\left[\partial_{r_{*}} \bar{h}_{1, n}^{(i)}\right]_{0} } & =\frac{4}{f_{0}} P_{\delta, n}^{(i)}-\frac{4}{f_{0}^{2}}\left[f_{0} \mathcal{S}_{1, n}^{(i)}+f_{0}^{\prime} \mathcal{T}_{1, n}^{(i)}\right] e^{-i \omega_{n m} \tau},
\end{aligned}
$$

where $P_{\delta, n}^{(i)}$ are the terms $\propto \delta\left(r-r_{0}\right)$ in $P_{1, n}^{(i)}$, which are given by

$$
\begin{aligned}
P_{\delta, n}^{(1)} & =\frac{1}{2} f_{0}^{2} f_{0}^{\prime}\left[\bar{h}_{1, n}^{(3)}\right]_{0}, \\
P_{\delta, n}^{(2)} & =\frac{1}{2} f_{0} f_{0}^{\prime}\left[f_{0} \bar{h}_{1, n}^{(3)}+\bar{h}_{1, n}^{(2)}-\bar{h}_{1, n}^{(1)}\right]_{0}, \\
P_{\delta, n}^{(4)} & =\frac{1}{4} f_{0} f_{0}^{\prime}\left[\bar{h}_{1, n}^{(4)}-\bar{h}_{1, n}^{(5)}\right]_{0}, \\
P_{\delta, n}^{(8)} & =\frac{1}{4} f_{0} f_{0}^{\prime}\left[\bar{h}_{1, n}^{(8)}-\bar{h}_{1, n}^{(9)}\right]_{0}, \\
P_{\delta, n}^{(i)} & =0 \quad \text { (others). }
\end{aligned}
$$

We may now easily obtain jump conditions for the second and higher $r_{*}$ derivatives in a recursive manner: Formally differentiating Eq. (F16) $k$ times with respect to $r_{*}$ we get the jump relations

$$
\begin{aligned}
{\left[\partial_{r_{*}}^{k+2} \bar{h}_{1, n}^{(i)}\right]_{0} } & =\left[\partial_{t}^{2} \partial_{r_{*}}^{k} \bar{h}_{1, n}^{(i)}\right]_{0}+4\left[\partial_{r_{*}}^{k} P_{1, n}^{(i)}\right]_{0} \\
& =-\left(\omega_{n m} f_{0} / \mathcal{E}_{0}\right)^{2}\left[\partial_{r_{*}}^{k} \bar{h}_{1, n}^{(i)}\right]_{0}+4\left[\partial_{r_{*}}^{k} P_{1, n}^{(i)}\right]_{0} \quad(k \geq 0),
\end{aligned}
$$


where the second equality is due to the harmonic dependence of $\bar{h}_{1, n}^{(i)}$ on $t$ along the orbit, expressed in Eq. (F15). The jumps in the $t$ derivatives are also obtained in a straightforward manner, by writing

$$
\left[\partial_{t}^{k} \partial_{r_{*}}^{k^{\prime}} \bar{h}_{1, n}^{(i)}\right]_{0}=\left(-i \omega_{n m} f_{0} / \mathcal{E}_{0}\right)^{k}\left[\partial_{r_{*}}^{k^{\prime}} \bar{h}_{1, n}^{(i)}\right]_{0}
$$

The desired jump conditions in $\bar{h}_{1}^{(i)}$ and their derivatives are finally obtained by adding up the jumps in the two $n=1$ harmonics:

$$
\left[\partial_{t}^{k} \partial_{r_{*}}^{k^{\prime}} \bar{h}_{1}^{(i)}\right]_{0}=\sum_{n= \pm 1}\left[\partial_{t}^{k} \partial_{r_{*}}^{k^{\prime}} \bar{h}_{1, n}^{(i)}\right]_{0} \quad\left(k, k^{\prime} \geq 0\right)
$$

[1] Y. Mino, M. Sasaki and T. Tanaka, Phys. Rev. D 55, 3457 (1997) arXiv:gr-qc/9606018.

[2] T. C. Quinn and R. M. Wald, Phys. Rev. D 56, 3381 (1997) arXiv:gr-qc/9610053.

[3] E. Poisson, Living Rev. Rel. 7, 6 (2004) arXiv:gr-qc/0306052.

[4] S. E. Gralla and R. M. Wald, Class. Quant. Grav. 25, 205009 (2008) arXiv:0806.3293 [gr-qc]].

[5] A. Pound, Phys. Rev. D 81, 024023 (2010) arXiv:0907.5197] [gr-qc]].

[6] L. Barack, Class. Quant. Grav. 26, 213001 (2009) arXiv:0908.1664 [gr-qc]].

[7] L. M. Burko, Phys. Rev. Lett. 84, 4529 (2000) arXiv:gr-qc/0003074.

[8] L. Barack and L. M. Burko, Phys. Rev. D 62, 084040 (2000) arXiv:gr-qc/0007033.

[9] S. Detweiler, E. Messaritaki and B. F. Whiting, Phys. Rev. D 67, 104016 (2003) arXiv:gr-qc/0205079.

[10] L. M. Diaz-Rivera, E. Messaritaki, B. F. Whiting and S. Detweiler, Phys. Rev. D 70, 124018 (2004) arXiv:gr-qc/0410011.

[11] L. Barack and D. A. Golbourn, Phys. Rev. D 76, 044020 (2007) arXiv:0705.3620 [gr-qc]].

[12] R. Haas, Phys. Rev. D 75, 124011 (2007) arXiv:0704.0797 [gr-qc]].

[13] I. Vega and S. Detweiler, Phys. Rev. D 77, 084008 (2008) arXiv:0712.4405 [gr-qc]].

[14] L. Barack and C. O. Lousto, Phys. Rev. D 66, 061502 (2002) arXiv:gr-qc/0205043.

[15] L. Barack and N. Sago, Phys. Rev. D 75, 064021 (2007) arXiv:gr-qc/0701069.

[16] T. S. Keidl, J. L. Friedman and A. G. Wiseman, Phys. Rev. D 75, 124009 (2007) arXiv:gr-qc/0611072.

[17] S. Detweiler, Phys. Rev. D 77, 124026 (2008) arXiv:0804.3529 [gr-qc]].

[18] M. V. Berndtson, arXiv:0904.0033 [gr-qc].

[19] N. Sago, L. Barack and S. Detweiler, Phys. Rev. D 78, 124024 (2008) arXiv:0810.2530 [gr-qc]].

[20] L. Blanchet, S. Detweiler, A. L. Tiec and B. F. Whiting, arXiv:0910.0207 [gr-qc].

[21] T. Damour, Phys. Rev. D 81, 024017 (2010) arXiv:0910.5533 [gr-qc]].

[22] L. Barack and C. Cutler, Phys. Rev. D 69, 082005 (2004) arXiv:gr-qc/0310125.

[23] R. Haas, presentation at the 11th Capra meeting, Orleans, June 2008.

[24] A. Pound and E. Poisson, Phys. Rev. D 77, 044013 (2008) arXiv:0708.3033 [gr-qc]].

[25] T. Hinderer and E. E. Flanagan, Phys. Rev. D 78, 064028 (2008) arXiv:0805.3337 [gr-qc]].

[26] L. Barack and C. O. Lousto, Phys. Rev. D 72, 104026 (2005) arXiv:gr-qc/0510019.

[27] L. Barack and A. Ori, Phys. Rev. D 61, 061502 (2000) arXiv:gr-qc/9912010.

[28] L. Barack, Phys. Rev. D 64, 084021 (2001) arXiv:gr-qc/0105040.

[29] L. Barack, Y. Mino, H. Nakano, A. Ori and M. Sasaki, Phys. Rev. Lett. 88, 091101 (2002) arXiv:gr-qc/0111001.

[30] L. Barack and A. Ori, Phys. Rev. D 67, 024029 (2003) arXiv:gr-qc/0209072.

[31] L. Barack, A. Ori and N. Sago, Phys. Rev. D 78, 084021 (2008) [arXiv:0808.2315 [gr-qc]].

[32] J. L. Barton, D. J. Lazar, D. J. Kennefick, G. Khanna and L. M. Burko, Phys. Rev. D 78, 064042 (2008) arXiv:0804.1075 $[\mathrm{gr}-\mathrm{qc}]]$.

[33] L. Barack and N. Sago, Phys. Rev. Lett. 102, 191101 (2009) arXiv:0902.0573 [gr-qc]].

[34] C. O. Lousto, M. Campanelli and Y. Zlochower, arXiv:0904.3541 [gr-qc].

[35] C. O. Lousto, H. Nakano, Y. Zlochower and M. Campanelli, arXiv:0910.3197] [gr-qc].

[36] A. Pound, E. Poisson and B. G. Nickel, Phys. Rev. D 72, 124001 (2005) arXiv:gr-qc/0509122.

[37] A. Pound and E. Poisson, Phys. Rev. D 77, 044012 (2008) arXiv:0708.3037 [gr-qc|].

[38] C. Cutler, D. Kennefick and E. Poisson, Phys. Rev. D 50, 3816 (1994).

[39] L. Barack and A. Ori, Phys. Rev. D 64, 124003 (2001) arXiv:gr-qc/0107056.

[40] S. Detweiler and B. F. Whiting, Phys. Rev. D 67, 024025 (2003) arXiv:gr-qc/0202086].

[41] Y. Mino, Phys. Rev. D 67, 084027 (2003) arXiv:gr-qc/0302075.

[42] C. O. Lousto, Class. Quant. Grav. 22, S543 (2005) arXiv:gr-qc/0503001].

[43] L. Barack, G. A. Golbourn and N. Sago, in preparation.

[44] K. Martel, Phys. Rev. D 69, 044025 (2004) arXiv:gr-qc/0311017.

[45] E. Poisson, Phys. Rev. D 70, 084044 (2004) arXiv:gr-qc/0407050.

[46] T. Tanaka, Prog. Theor. Phys. 90, 65 (1993).

[47] R. Fujita, W. Hikida and H. Tagoshi, Prog. Theor. Phys. 121, 843 (2009) arXiv:0904.3810 [gr-qc]].

[48] Fujita et al.'s flux data in Table $\mathrm{V}$ were provided to us by R. Fujita (private communication). 
[49] K. Glampedakis and D. Kennefick, Phys. Rev. D 66, 044002 (2002) arXiv:gr-qc/0203086].

[50] A. Buonanno and T. Damour, Phys. Rev. D 62, 064015 (2000) arXiv:gr-qc/0001013.

[51] A. Ori and K. S. Thorne, Phys. Rev. D 62, 124022 (2000) arXiv:gr-qc/0003032.

[52] T. Damour, P. Jaranowski and G. Schaefer, Phys. Rev. D 62, 084011 (2000);

[53] L. Blanchet and B. R. Iyer, Class. Quant. Grav. 20, 755 (2003) arXiv:gr-qc/0209089.

[54] J. Thornburg, arXiv:0909.0036 [gr-qc].

[55] L. Barack and S. R. Dolan, in preparation.

[56] P. Canizares and C. F. Sopuerta, Phys. Rev. D 79, 084020 (2009) arXiv:0903.0505 [gr-qc]].

[57] S. E. Field, J. S. Hesthaven and S. R. Lau, Class. Quant. Grav. 26, 165010 (2009) arXiv:0902.1287 [gr-qc]].

[58] L. Barack, T. Damour and N. Sago (work in progress).

[59] C. O. Lousto and H. Nakano, Class. Quant. Grav. 25, 145018 (2008) arXiv:0802.4277 [gr-qc]].

[60] I. Vega, P. Diener, W. Tichy and S. Detweiler, Phys. Rev. D 80, 084021 (2009) arXiv:0908.2138 [gr-qc]].

[61] L. Barack, D. A. Golbourn and N. Sago, Phys. Rev. D 76, 124036 (2007) arXiv:0709.4588 [gr-qc]].

[62] N. J. Warburton and L. Barack, in preperation.

[63] The applicability of Eq. (2.33) relies on the validity of the symmetry relation (2.32); the latter holds whenever the orbit is periodic with one intrinsic frequency. This is the case for all bound geodesics in Schwarzschild, and also for equatorial (and possibly eccentric) or circular (and possibly inclined) orbits in Kerr. However, the symmetry relation and Eq. (2.33) do not apply in the Kerr case when the orbit is both eccentric and inclined.

[64] Although Martel's expressions for the horizon fluxes in [44] are correct, his derivation of these fluxes was not; this was later noted and corrected by Poisson in [45].

[65] Interestingly, we reproduced the linear zoom-whirl behavior with a manipulated scalar-field model, where the peak of the scalar-field's effective potential is artificially "shifted" to radii below the whirl radius. This behavior deserves further investigation, but we have not attempted such an investigation here. 